\title{
Surfactant Flow between a Plateau Border and a Film during Foam Fractionation
}

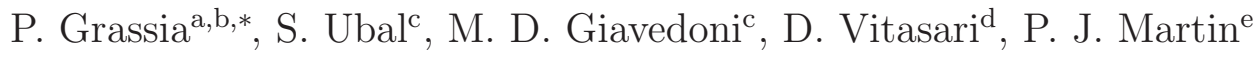 \\ ${ }^{a}$ Dept of Chemical and Process Engineering, University of Strathclyde, \\ James Weir Building, 75 Montrose St G1 1XJ, UK \\ ${ }^{b}$ Departamento Ciencias Matemáticas y Físicas, Universidad Católica de Temuco, \\ Rudecindo Ortega 02950, Temuco, Chile \\ ${ }^{c}$ UNL CONICET, INTEC, Güemes 3450, 3000 Santa Fe, Argentina \\ ${ }^{d}$ Dept Chemical Engineering, Universitas Muhammadiyah Surakarta, \\ Jl A Yani Tromol Pos 1 Pabelan, Surakarta 57162, Indonesia \\ ${ }^{e}$ CEAS, The Mill, University of Manchester, Oxford Rd, Manchester M13 9PL, UK
}

\begin{abstract}
A fluid mechanics problem relevant to foam fractionation processes is analysed. Specifically the fluid flow field transporting surfactant from foam Plateau borders (fed with surfactant-rich material) towards comparatively surfactant-lean foam films is considered. The extent to which this surfactant mass transfer is limited by surface viscous effects is studied. Previous work (Vitasari et al., 2015) made assumptions about the likely flow field along the Plateau border surface. These assumptions suggested that 'high' surface viscosity (measured by a suitable dimensionless parameter) led to strong suppression of the rate of surfactant mass transfer from Plateau border to film, whereas 'low' surface viscosity did not suppress this mass transfer rate in any significant way. More detailed fluid mechanical calculations which are carried out here corroborate the aforementioned assumptions in the 'high' surface viscosity regime. However the calculations suggest that in the 'low' surface viscosity regime, in contrast to the findings from the previous assumptions, moderate reductions in the rate of surfactant mass transfer are also possible. Counterintuitively these moderate reductions in mass transfer rate potentially have more negative impact on fractionation processes than the aforementioned strong suppression. This is because they tend to arise under conditions for which the efficiency of the fractionation system is particularly sensitive to any reduction whatsoever in the surfactant
\end{abstract}

\footnotetext{
*Corresponding author

Email address: paul.grassia@strath.ac.uk (P. Grassia)
} 
mass transfer rate.

Keywords: Mathematical Modelling; Computational Fluid Dynamics; Interfacial Rheology; Surfactant; Bubble; Films

\section{Highlights}

* Fluid mechanics of foam fractionation in reflux and/or stripping mode is modelled

* Surfactant transfers from surfactant-rich Plateau borders to surfactant-lean films

* Effect of surfactant surface viscosity on flow in Plateau border is analysed

* High surface viscosity strongly suppresses border to film mass transfer

* Even low surface viscosities can reduce surfactant mass transfer onto foam films

\section{Introduction}

The purpose of foam fractionation is to enrich surfactants or surface active materials by allowing them to accumulate on foam films (Lemlich, 1968a,b). During this process, whilst surfactant can be transported to foam film surfaces diffusively (Vitasari et al., 2013a), transport rates can be enhanced by exploiting so called Marangoni flows (Vitasari et al., 2013b), which rely on surface tension differences driving convection. There are various foam fractionation scenarios (described more fully below) where such convective Marangoni flows are likely to arise. These all involve comparatively surfactant-lean films being surrounded by surfactant-rich Plateau borders (tricuspid channels along which three films meet). The Plateau borders typically contain rather more liquid (and thus potentially more surfactant) than the foam films, and so can feed surfactant to those films via convective Marangoni flows.

One relevant scenario is that of foam fractionation with reflux (Brunner and Lemlich, 1963; Lemlich and Lavi, 1961; Martin et al., 2010; Stevenson and Jameson, 2007). During this process, which can be operated either batchwise or continuously, foam collected from the top of a fractionation column is collapsed and the liquid recovered is poured back onto the column. This liquid then flows downward through the Plateau borders which form a network of channels. The cross sectional area of the channels is set by 
the liquid flux through them (Weaire and Hutzler, 1999), with the dominant force balance (Grassia et al., 2001) in these channels being between gravity (acting downwards) and viscous drag at the Plateau border walls (the drag acting upwards on the downward flowing liquid). This downward flowing liquid added back to the column provides an additional opportunity to increase the surfactant content in the foam. Various mass exchange mechanisms are expected to take place, including the surfactant-rich reflux liquid mixing with less surfactant-rich liquid already in the Plateau borders lower down in the column, in addition to the mechanism of main interest here, namely Marangoni flows pulling surfactant-rich material onto surfactant-lean films.

How effective the Marangoni mechanism is in this context depends on the concentration regime in which the fractionation process is being operated. Surface tension loses sensitivity to surfactant concentration at a critical surfactant concentration (the critical micelle concentration or CMC (Chang and Franses, 1995)) considered to be the point at which the surface is sufficiently crowded with surfactant that excess surfactant begins to form aggregates (or micelles) in the bulk. Marangoni mechanisms are unlikely to be effective at increasing surfactant coverage on an already crowded surface, but should be effective at concentrations below the CMC. Exceedingly low concentration operation (with barely any surface coverage of surfactant) will be problematic for foam stability: in such a situation however, reflux (and the Marangoni-driven surfactant mass transfer it induces) will help to stabilise the foam films.

Another scenario of relevance here is fractionation in stripping mode (Lemlich, 1968a). During a continuous stripping operation, liquid feed is introduced part-way up the column (instead of to a liquid pool underneath the foam) and (as with fractionation employing reflux) drains downward through the Plateau border network, again with the channel cross sections being determined by the liquid flux. Given that the purpose of the process is to strip as much surfactant as possible from the feed stream, even if the feed concentration is above the CMC, at some point lower down in the column it should fall below the CMC. The Marangoni-driven mass transfer processes described above will then become active pulling material onto (comparatively uncrowded) foam films. 
Yet another potentially relevant scenario is multicomponent foam fractionation (Brown et al., 1999). Such a system involves two surfactants: a more surface active one and a less surface active one. Marangoni flows onto films are expected if a more surface active species in a Plateau border contacts a film covered with less active species (but not the other way round). If (as could happen in stripping mode multicomponent operation) the feed to the Plateau border network contains a mixture of two surface active species, the Marangoni flows themselves are unlikely to be selective, carrying both species of the mixture onto the film. However the less active species is more likely to desorb to the interior of the film (Vitasari et al., 2013a). Drainage flows in the film interior might then carry that species back towards the Plateau border (Vitasari et al., 2013b) and after that downwards to the liquid pool underneath the foam.

In view of the importance of Marangoni flows to the above mentioned fractionation scenarios, recently a model has been developed (Vitasari et al., 2013b) for the Marangonidriven surfactant accumulation on foam films. It is expected however that (over and above Marangoni mechanisms) additional interfacial rheology effects (e.g. surface viscosity (Scriven, 1960)) should affect surfactant convection onto foam films. Hence the surfactant accumulation model of Vitasari et al. (2013b) was extended (Vitasari et al., 2015) to incorporate the surface viscosity (in addition to the viscous effects in the bulk of the films which were included in the models from the outset). The basic finding was that (unsurprisingly) the presence of surface viscosity tends to limit the surfactant movement and hence can reduce the rate of surfactant accumulation on the films.

It is important to note that the model of Vitasari et al. (2015, 2013b) is mathematically very simple. As explained in Vitasari et al. (2015), it consists of a 1-d ordinary differential equation for the surface velocity on the foam film surface, coupled to a partial differential equation (in terms of time and one spatial dimension) for the evolution of the surfactant concentration. The model was therefore sufficiently simple (and hence sufficiently quick to solve, typical run times being just a few minutes) that it is feasible to incorporate it into design algorithms for fractionation columns.

In order to solve the model, it was necessary (Vitasari et al., 2015) to make assumptions 
(described in more detail later) about how the flow on the film matched onto that in the Plateau border. The assumptions made were plausible, but nevertheless need to be checked by more detailed fluid mechanical calculations. The objective of the present work then is to perform fluid mechanical calculations to check the modelling assumptions of Vitasari et al. (2015) and if necessary indicate how to modify these assumptions.

The remainder of this discussion is laid out as follows. Section 2 sets up governing equations for the fluid flow field on a film employing simple lubrication theory approximations. Section 3 then sets up corresponding governing equations for the fluid flow in the Plateau border (considering both a 2-d flow field and a quasi-1-d asymptotic approach) whilst Section 4 considers matching between the film and the border. Section 5 identifies and estimates the values of a number of key dimensionless groups. This section also includes a discussion of how the values of these dimensionless groups are believed to affect the solution for the flow field. Section 6 considers a special case where we are able to solve analytically for the flow field in the film: this shows directly how the nature of the filmPlateau border coupling influences the film flow field (and thereby the surfactant mass transfer rate from border to film). Next Section 7 describes the numerical methodology for solving the Plateau border flow fields with results presented in Section 8. Section 9 discusses and summarises the results. Finally overall conclusions are given in Section 10.

\section{Model for film flow}

Figure 1(a) shows a sketch of a film joining up to a Plateau border. In the first instance we focus on the flow in the film. We adopt the model already considered by Vitasari et al. (2015) in which the film is taken to be perfectly flat (i.e. we ignore the complications associated with possible dimpled shapes of the film near its junction with the Plateau border (Frankel and Mysels, 1962; Joye et al., 1992, 1994, 1996)). The film is also assumed not to drain so its thickness is taken to be constant as well as uniform (although effects of film drainage causing thickness to vary with time could be incorporated into the model if desired (Vitasari et al., 2013b); film Marangoni flows tend to start off dominating film drainage flows, but the Marangoni flows also decay more rapidly as surfactant is 
transported onto the film surface, and film drainage might then be a significant contributor to any remaining transport (Vitasari et al., 2013b)). Moreover the film thickness is much smaller than its length (the half-thickness being denoted $\delta_{0}$ and the half-length being denoted $L$ with $\left.\delta_{0} \ll L\right)$ : lubrication theory approximations apply. These give a parabolic flow profile across the film thickness as sketched in Figure 1(b)

$$
u=u_{s}(x)\left(\frac{3}{2} \frac{y^{2}}{\delta_{0}^{2}}-\frac{1}{2}\right)
$$

where $x$ is a coordinate along the film (with $x=0$ corresponding to the point where the film meets the Plateau border, and defined such that $x<0$ on the film), $y$ is a coordinate across the film (with $y=0$ midway across), $u$ is the velocity in the film, $u_{s}$ is the velocity on the film surface, and $\delta_{0}$ is the film half-thickness.

This profile implies a viscous shear stress at the film surface

$$
\mu \partial u /\left.\partial y\right|_{y=\delta_{0}}=3 \mu u_{s} / \delta_{0}
$$

where $\mu$ is viscosity of the liquid in the film. On the gas-liquid surface we then have (matching the shear stress to Marangoni and surface viscous stresses)

$$
\mu \partial u /\left.\partial y\right|_{y=\delta_{0}}=\partial \gamma / \partial x+\mu_{s} \partial^{2} u_{s} / \partial x^{2}
$$

where $\gamma$ is surface tension and $\mu_{s}$ is surface viscosity. Substituting (2) into (3) gives

$$
3 \mu u_{s} / \delta_{0}=\partial \gamma / \partial x+\mu_{s} \partial^{2} u_{s} / \partial x^{2}
$$

The question of interest here is, given an instantaneous distribution of $\gamma$ vs $x$, what is the value of $\left.u_{s}\right|_{x=0}$ ? This quantity is of interest because $\left.u_{s}\right|_{x=0}$ governs the flow and hence mass transfer between Plateau border and film (and hence the time evolution of $\gamma$ ).

In order to determine $\left.u_{s}\right|_{x=0}$, equation (4) needs to be solved subject to suitable boundary conditions. On symmetry grounds, $u_{s}$ vanishes at the centre of the surface of the film. Again on symmetry grounds, $u_{s}$ vanishes at the centre of the surface of the 
Plateau border: see Figure 1(a). Given that we have one condition on the film, and one condition on the border (with equation (4), as formulated above, applying only to the film), we need somehow to match the film flow to the border flow in order to solve for $u_{s}$.

The approach of Vitasari et al. (2015) was to assume that the velocity field $u_{s}(x)$ on the film could be 'extrapolated' onto the border. Matching was achieved by 'unfolding' the surface of the border onto a straight line, taking a uniform surface strain rate (and hence a uniform $\partial u_{s} / \partial x$ equal to the value $\partial u_{s} /\left.\partial x\right|_{x=0}$ at the junction with the film) on the now 'unfolded' border. The question we plan to address is whether or not this assumption used by Vitasari et al. (2015) to achieve matching was appropriate or not.

\section{Flow in a Plateau border}

The complete set of fluid mechanical calculations required to check the aforementioned assumptions of Vitasari et al. (2015) turn out to be rather detailed and complicated ones. The reasons for these complications (and a possible way around them, which we employ in this work) are outlined below.

Conventionally one thinks of the surface of a Plateau border as being highly curved by comparison with the films (see e.g. Figure 1(a)). Films must meet threefold at Plateau borders at $2 \pi / 3$ angles, but since cross-sections of Plateau borders (for a dry foam at least) are much smaller than lengths of films, sharp curvatures at the borders are required so as to turn through these $2 \pi / 3$ angles over a comparatively small length scale.

Idealising, the curved Plateau border surfaces are treated as being arcs of circles, whilst films are treated as flat. In a foam, the pressure difference (Weaire and Hutzler, 1999) (or more correctly, the normal stress difference) between the gas in the bubbles and the liquid in either Plateau borders or films depends on curvature of the gas-liquid interface (the Young-Laplace law). Since the borders are highly curved, they tend to have lower pressure than the films, meaning that liquid can drain from the films into the borders. Over and above this, in the application of interest here (a fractionation column operated e.g. with reflux and/or in stripping mode) there is a flux of liquid added to the foam, which flows through the Plateau borders swelling them. The result of all of this 
is that borders tend to contain rather more liquid than films: even though the border cross-section is smaller than the lengths of the films, the film thicknesses can be very tiny indeed, and hence the total amount of liquid in the films can be small ${ }^{1}$.

In fluid flow problems involving films joining up with Plateau borders, pressure must change continuously with position moving from the films into the borders. The complication of course is that the curvature of the liquid-gas interfaces is tied to pressure difference: the picture whereby borders are uniformly curved and films are flat is not strictly valid. What one can observe in the neighbourhood of where the film meets the border is a so called 'transition region' (a concept introduced by Bretherton (1961) and discussed also by Reinelt and Kraynik (1989, 1990); Schwartz and Princen (1987)), with pressures and curvatures varying significantly over a comparatively small distance near that film-Plateau border junction. Away from the transition region, the film can be thought of as comparatively flat (i.e. zero curvature) and likewise, the border can be thought of as a circular arc of uniform curvature. In the transition region neither of these two situations applies. The distribution of curvature along the interfaces determines their position, which in turn defines the solution domain where liquid is present. This domain itself must strictly speaking be obtained as a part of the solution of a free boundary problem, alongside the fluid flow fields themselves. Fluid flows between films and Plateau borders can produce complex interface shapes (e.g. dimpled shapes ${ }^{2}$ as referred to earlier (Frankel and Mysels, 1962; Joye et al., 1992, 1994, 1996)). Adding surface rheological effects (Marangoni and surface viscous forces) to the above description complicates matters even further. Compared to the simple and quick-to-solve models studied by Vitasari et al. (2015, 2013b) (as already alluded to above), such complex fluid mechanical calculations are less readily incorporated into design algorithms for fractionation columns.

The question we wish to ask here is whether there might be some way to explore the validity (or otherwise) of the aforementioned 'extrapolation' and 'unfolding' assumptions

\footnotetext{
${ }^{1}$ For a stable foam, the films, when they eventually become thin enough, are stabilised by colloidal disjoining pressures and film drainage then stops.

${ }^{2}$ Note that colloidal disjoining forces are also neglected here, but these can become relevant in exceedingly thin films, and might tend to counteract dimpling.
} 
used by Vitasari et al. (2015) for flow fields on Plateau border surfaces without resorting to the full complexity of a free boundary problem.

The suggestion (analogous to what has been done in some other surface rheology driven flows (Grassia and Homsy, 1998a,b; Smith and Davis, 1983)) is to solve a fluid flow problem in a known domain, in this particular case where the entirety of the surfaces of the Plateau borders are treated as uniform curvature arcs (see Figure 1(a)), ignoring transition regions, dimpling, etc.. We permit tangential motion along the Plateau border surfaces (tangential motion is essential to permit mass exchange between border and film) but no normal motion. The shape of the flow domain is then not only specified, but also is constant over time. The shape of the flow domain is by assumption unaffected by dynamic parameters such as e.g. capillary number which otherwise would govern the surface shape in systems of this type (Bretherton, 1961). With the aid of computational fluid dynamics simulations, it is now possible to determine the flow fields throughout the border and, specifically at the point where the border meets the film, check for consistency with the assumptions employed by Vitasari et al. (2015).

In addition to flow fields, these computational fluid dynamics calculations can also access pressure fields. Note that the computed pressure (more correctly the computed normal stress) will in general be non-uniform along the border surface whereas curvature is (by assumption) uniform. Such a situation violates the Young-Laplace law, but the level of non-uniformity in the computed pressure profile is informative: zones where the pressure deviates most strongly from uniformity are also zones where the assumption of constant border curvature is least tenable.

The remainder of this section is laid out as follows. Section 3.1 gives the governing equations for the Plateau border flow field with boundary conditions given in Sections 3.23.4. Following that, Section 3.5 contains an asymptotic 'quasi-1-dimensional' description of the flow fields as an alternative to the fully 2-d formulation of Section 3.1-Section 3.4.

\subsection{Governing equations for the 2-D flow field in the Plateau border}

The flow domain for the tricuspid Plateau border is shown in Figure 2. We only need to describe one sixth of the tricuspid domain, the rest following on symmetry grounds. As 
noted by Leonard and Lemlich (1965) the domain is most conveniently described in polar coordinates. The angular coordinate $\theta$ satisfies $0 \leq \theta \leq \frac{\pi}{6}$, and the radial coordinate $r$ satisfies $a \leq r \leq a\left(1+\Delta_{0}\right) / \cos \theta$, where $a$ is the curvature radius of the Plateau border and $\Delta_{0}$ is the ratio between film half-thickness $\delta_{0}$ and Plateau border curvature radius $a$. Note that $\Delta_{0}$ is a small parameter on the grounds that the film is exceedingly thin.

We assume incompressible Stokes flow in the Plateau border with a pressure field $P$, a velocity field $\boldsymbol{u}$, and a liquid viscosity $\mu$. Hence the Plateau border flow satisfies

$$
\begin{gathered}
\nabla \cdot \boldsymbol{u}=0 \\
0=-\nabla P+\mu \nabla^{2} \boldsymbol{u} .
\end{gathered}
$$

Note that even though we are considering a Stokes flow with the same flow domain as Leonard and Lemlich (1965), we are dealing with quite distinct flows. The work of Leonard and Lemlich (1965) treated unidirectional flow directed normal to the plane of Figure 2. Here we consider flow in the plane of Figure 2. We now proceed to consider boundary conditions on the various domain boundaries.

\subsection{Plateau border surface}

As we are dealing with a system involving interfacial rheology, the most important boundary is the gas-liquid surface of the Plateau border, $r=a$ in our coordinate system.

As was mentioned earlier (see Section 3) in the interests of simplicity, we focus on a problem where the solution domain is fixed. Hence the radial velocity component $u_{r}$ on the gas-liquid boundary is assumed to vanish.

Regarding tangential velocities, we use the symbol $u_{s}$ to denote the velocity component $u_{\theta}$ evaluated at the Plateau border surface. We also use the variable $s$ to denote the distance measured along the Plateau border surface, defined by $s=a \theta$. Ultimately $u_{s}$ is determined via a tangential boundary condition (incorporating surface viscosity on the gas-liquid surface) which is as follows:

$$
-\mu r \frac{\partial}{\partial r}\left(\frac{u_{\theta}}{r}\right)=\frac{\partial \gamma}{\partial s}+\mu_{s} \frac{\partial^{2} u_{s}}{\partial s^{2}},
$$


the notation here recognising that $u_{\theta}$ depends on $r$ as well as upon $\theta$, whereas $u_{s}$ depends on $s$ (but not upon $r$ ). This is a generalisation of equation (3) given previously, the sign of the first term recognising that the outward normal to the Plateau border points here in the direction of decreasing $r$.

\subsection{Plateau border entrance}

We now specify the boundary condition across the thickness at the Plateau border entrance $\theta=0$. The tangential velocity profile $u_{\theta}$ vs $r$ across the entrance is inherited from the film. Analogously to equation (1) a parabolic profile is assumed

$$
u_{\theta}=\left(-\frac{3}{2} \frac{\left(1+\Delta_{0}-a^{-1} r\right)^{2}}{\Delta_{0}^{2}}+\frac{1}{2}\right)\left|u_{s}\right|_{s=0} \mid
$$

Note the use of an absolute value on the right hand side of (8): we anticipate that the sign of $\left.u_{\theta}\right|_{r=a, \theta=0}$ (which by definition is equal to $\left.u_{s}\right|_{s=0}$ and equivalently to $\left.u_{s}\right|_{x=0}$ ) is negative, implying surfactant transfer from Plateau border to film (the direction of transfer expected in a fractionation column when film surfaces are fed by Marangoni flows).

To solve for the Plateau border flow field, a second boundary condition is however required on $\theta=0$. Identifying an appropriate condition that matches the Plateau border onto the film in a sensible fashion is subtle for reasons explained in the appendix: we elected to adopt a tangential stress condition, setting the $r, \theta$ component of the stress equal to $\mu \partial u_{\theta} / \partial r$, the value of $\partial u_{\theta} / \partial r$ being determined from equation (8).

\subsection{Symmetry lines}

In addition to the boundary conditions discussed in Sections 3.2-3.3, we also need to impose boundary conditions on two symmetry lines represented in polar coordinates by $\theta=\pi / 6$ (with varying $r$ ) and $r=a\left(1+\Delta_{0}\right) / \cos \theta$ (with varying $\theta$ ).

We use $\boldsymbol{n}$ and $\boldsymbol{t}$ to represent the unit outward normal vector and unit tangent, and $u_{n}$ and $u_{t}$ to represent normal and tangential velocity components. The boundary conditions required are $\boldsymbol{u} \cdot \boldsymbol{n}=0$ and $\boldsymbol{n} . \nabla(\boldsymbol{u} . \boldsymbol{t})=0$, or expressed more simply $u_{n}=0$ and $\boldsymbol{n} . \nabla u_{t}=0$. 


\subsection{Quasi-1-d asymptotic approach for the Plateau border}

In addition to the 2-d formulation alluded to above, there is also a comparatively simple 'quasi-1-d' asymptotic approach. This describes the region of the Plateau border that is close to the film as sketched in Figure 1(b).

We have Cartesian coordinate $x=0$ at the junction between the film and the Plateau border and $x>0$ in the border itself. Restricting attention to the region $x \ll a$ we can employ a lubrication theory entirely analogous to that in Section 2 for the film. The velocity profile across the border is

$$
u \approx u_{s}(x)\left(\frac{3}{2} \frac{y^{2}}{(\delta(x))^{2}}-\frac{1}{2}\right)
$$

where $\delta$ is now the half border thickness (which in the border varies with longitudinal position $x$ ). Near the entrance to the border we have

$$
\delta \approx \delta_{0}+\frac{1}{2} \frac{x^{2}}{a}
$$

which upon defining $\Delta=\delta / a$ becomes $\Delta \approx \Delta_{0}+\frac{1}{2} x^{2} / a^{2}$. It is clear that for $x \ll a$, both $\Delta \ll 1$ and $\mathrm{d} \delta / \mathrm{d} x \ll 1$ (which are necessary conditions for lubrication theory to apply).

The analysis for this quasi-1-d formulation proceeds entirely analogously to Section 2, and indeed analogously to equation (4) we deduce

$$
3 \mu u_{s} / \delta=\partial \gamma / \partial x+\mu_{s} \partial^{2} u_{s} / \partial x^{2}
$$

Equation (11) must be solved with suitable boundary conditions. At $x=0$, the values of $u_{s}$ and $\partial u_{s} / \partial x$ must match up with those on the film. The other boundary condition that $u_{s}$ must vanish at the symmetry point at the centre of the Plateau border surface is slightly more problematic, because it is strictly speaking outside the domain $x \ll a$ for which equations (9)-(11) apply, a complication we will discuss in due course. Provided this complication can be overcome, equation (11) provides an alternative way to determine the surface velocity distribution $u_{s}$ vs $x$ or equivalently $u_{s}$ vs $s$ ( $s$ being arc length along 
the surface), instead of using the 2-d approach of Sections 3.1-3.4.

Regardless of which of the two approaches we adopt, we now introduce a simplification. We admit Marangoni stresses on the film (i.e. non-zero $\partial \gamma / \partial x$ on the film) but not on the Plateau border. Thus we set $\gamma$ to equal a constant value $\gamma_{0}$ on the Plateau border, so that $\partial \gamma / \partial x$ vanishes in equation (11) or equivalently $\partial \gamma / \partial s$ vanishes in equation (7).

The justification is that the Plateau border is typically much thicker than the (exceedingly thin) film, so its interior can act as a reservoir of surfactant. Even if the surfactantrich border loses material to the surfactant-lean film, the Plateau border's surface can be replenished by new surfactant arriving from its interior, provided this new surfactant manages to arrive at the Plateau border surface more quickly than it is lost, implying in turn assumptions about the kinetics of surface adsorption. Under circumstances whereby the Plateau border can act as a reservoir, there must be a physicochemical length scale, an 'effective Henry constant' (defined as the ratio between the concentration of surfactant adsorbed at interfaces and the concentration of surfactant in the bulk ${ }^{3}$ ) which we assume to be much larger than the film thickness, but smaller than the typical Plateau border thickness (which is comparable with the curvature radius of the Plateau border). Hence, despite there being negligible amounts of surfactant in solution within films, there are nevertheless significant amounts of surfactant in solution within the Plateau border.

Note that based on equation (10), the border is only substantially thicker than the film for $x \gg O\left(\sqrt{\delta_{0} a}\right)$ or equivalently for $x \gg O\left(\Delta_{0}^{1 / 2} a\right)$. Since $\Delta_{0} \ll 1$, this applies to the overwhelming majority of the border. For $x$ values up to order $O\left(\sqrt{\delta_{0} a}\right)$ however, the border is only marginally thicker than the film, and strictly speaking we should continue to account for Marangoni stresses there, even if they are neglected over the rest of the border. If however both $u_{s}$ and $\partial u_{s} / \partial x$ vary comparatively little between $x=0$ and $x=O\left(\Delta_{0}^{1 / 2} a\right)$, it is permissible to compute flow fields over the entire Plateau border ignoring Marangoni stresses once again over the entire border surface.

Neglecting Marangoni stresses on the border surface, implies that the flow in the border

\footnotetext{
${ }^{3}$ We use the expression, 'effective Henry constant', rather than simply 'Henry constant', to recognize that adsorbed surfactant might be a non-linear function of bulk surfactant concentration.
} 
is wholly driven by non-zero flow at the junction between the Plateau border and the film, i.e. due to $\left.u_{s}\right|_{x=0}$ (or equivalently $\left.u_{s}\right|_{s=0}$ ) being non-zero. Moreover we are dealing with a linear system of equations so that the ratio between $\partial u_{s} /\left.\partial x\right|_{x=0}$ and $\left.u_{s}\right|_{x=0}$ is independent of the value of $\left.u_{s}\right|_{x=0}$ : the ratio can be obtained even if $\left.u_{s}\right|_{x=0}$ is a priori unknown.

Both $\partial u_{s} / \partial x$ and $u_{s}$ are continuous at $x=0$ moving from the Plateau border to the film: hence, if we can determine their ratio on the Plateau border side of $x=0$ (without needing to compute the film flow), we immediately know their ratio on the film side also. Thus we have a 'matching condition' joining the Plateau border and film flows. Once this ratio or 'matching condition' is determined, the film flow can be solved without further details of the flow in the Plateau border, apart from the 'matching condition' itself.

Given this 'matching condition' is important for determining the film flow, there has been speculation (Vitasari et al., 2015) regarding what the ratio between $\partial u_{s} /\left.\partial x\right|_{x=0}$ and $\left.u_{s}\right|_{x=0}$ might be. Hypotheses for the value of this ratio are discussed in the next section.

\section{Matching conditions between Plateau border and film}

This section is laid out as follows. Section 4.1 considers one possible hypothesis from Vitasari et al. (2015) of how to match the Plateau border flow to that in the film. The consequences of this hypothesis for the film flows are identified in Section 4.2. An alternative matching hypothesis is proposed in Section 4.3. It is ultimately by performing fluid mechanical calculations in the Plateau border (considered later on) that we can identify conditions under which each hypothesis actually applies.

\subsection{Uniform strain rate matching hypothesis}

The study of Vitasari et al. (2015) speculated that

$$
\partial u_{s} /\left.\partial x\right|_{x=0}=-\left.u_{s}\right|_{x=0} /(\pi a / 6)
$$

This is consistent with a uniform surface rate of strain on the Plateau border, i.e. a uniform decay of velocity on the border surface 'extrapolated' from the entrance to the border (where it meets the film) to the border's symmetry point (the distance $\pi a / 6$ being 
the distance from the entrance to the symmetry point measured along the surface in the tricuspid Plateau border geometry, and we have 'unfolded' this curved surface onto a straight line along the direction of the film).

Assumption (12) turns out to be sufficient to close the set of equations for the flow on the film (Vitasari et al., 2015), enabling us to obtain $u_{s}$ at any given $x$, and in particular $\left.u_{s}\right|_{x=0}$. As mentioned previously, the velocity $\left.u_{s}\right|_{x=0}$ is very important here, as it is precisely this velocity that governs the transport of surfactant between border and film.

Some of the consequences of assumption (12) are explored in the next section.

\subsection{Film-Plateau border matching with a uniform strain rate hypothesis}

It can be shown (Vitasari et al., 2015) (independently of the assumption in equation (12)) that a velocity boundary layer can appear at the edge of the film as the Plateau border is approached: the velocity on the film surface changes quite rapidly with position within this boundary layer. Specifically if $u_{s}$ immediately outside the boundary layer is denoted $u_{s(o)}$, then the boundary layer approximation results in

$$
\partial u_{s} /\left.\partial x\right|_{x=0} \approx\left(\left.u_{s}\right|_{x=0}-u_{s(o)}\right) /\left(L \sqrt{\delta_{0}^{\prime} \bar{\mu}_{s} / 3}\right) \approx\left(\left.u_{s}\right|_{x=0}-u_{s(o)}\right) /\left(L \frac{\pi}{6} a_{c r i t}^{\prime}\right)
$$

where recall $L$ is the half-film length, and $\delta_{0}^{\prime}, \bar{\mu}_{s}$ and $a_{c r i t}^{\prime}$ are dimensionless groups defined as follows: $\delta_{0}^{\prime}$ is an aspect ratio $\left(\delta_{0} / L\right), \bar{\mu}_{s}$ is a dimensionless surface viscosity $\left(\mu_{s} /(\mu L)\right)$, and moreover $a_{c r i t}^{\prime}$ is

$$
a_{c r i t}^{\prime}=\frac{6}{\pi} \sqrt{\delta_{0}^{\prime} \bar{\mu}_{s} / 3}
$$

We also define a dimensionless Plateau border curvature radius $a^{\prime}$ to be $a / L$ (with $a^{\prime}<1$ on geometric grounds), interpreting $a_{c r i t}^{\prime}$ as being a critical value of $a^{\prime}$ at which the presence of the Plateau border starts to limit the film flow (further details to be given shortly).

In equation (13), the term $L \sqrt{\delta_{0}^{\prime} \bar{\mu}_{s} / 3}$ or equivalently $L \frac{\pi}{6} a_{c r i t}^{\prime}$ represents the length scale of the velocity boundary layer on the film. Substitution from the definitions of $\delta_{0}^{\prime}$ and $\bar{\mu}_{s}$, gives the boundary layer length as $\sqrt{\delta_{0} \mu_{s} /(3 \mu)}$. This depends on (dimensional) film thickness $\delta_{0}$ and on interfacial and bulk rheology (i.e. the ratio $\mu_{s} / \mu$ ), but not on the length scale of the film (half-length $L$ ) nor that of the border (curvature radius $a$ ). 
The boundary layer length scale determined here is also independent of how surface tension $\gamma$ vs $x$ varies, implying that the velocity field can have a boundary layer character even when the Marangoni stress field does not. There could be more complex situations for which both the velocity field and the Marangoni stress field develop boundary layers, hence modifying equation (13), although we neglect such complications here.

Substitution of equation (13) into equation (12) (which assumes uniform strain rate on the border as mentioned above) gives

$$
\left(\left.u_{s}\right|_{x=0}-u_{s(o)}\right) / a_{c r i t}^{\prime} \approx-\left.u_{s}\right|_{x=0} / a^{\prime} .
$$

Rearranging implies

$$
\left.u_{s}\right|_{x=0} \approx u_{s(o)}\left(1+a_{c r i t}^{\prime} / a^{\prime}\right)^{-1} \approx u_{s(o)}\left(1+\frac{6}{\pi} \sqrt{\frac{\Delta_{0} M_{s}}{3}}\right)^{-1}
$$

where $\Delta_{0}$ is $\delta_{0}^{\prime} / a$ and where $M_{s}$ is a (rescaled) dimensionless surface viscosity defined as $M_{s}=\mu_{s} /(\mu a)$, a parameter already identified by Leonard and Lemlich (1965).

It is clear from this result that $\left.u_{s}\right|_{x=0}$ can be more or less the same magnitude as $u_{s(o)}$ (i.e. the change in velocity $u_{s}$ across the boundary layer is insignificant) if $a^{\prime} \gg a_{c r i t}^{\prime}$. This is a situation where the Plateau border is sufficiently big, and/or the foam liquid fraction (dominated by the liquid in the borders) is sufficiently high, and/or the surface viscosity is sufficiently weak, that the film is 'unaware' of any constraints arising from the presence of the Plateau border: in particular, the film flow field is unaffected by the constraint that velocity must vanish at the symmetry point of the Plateau border.

If however $a^{\prime} \ll a_{c r i t}^{\prime}$, then $\left.u_{s}\right|_{x=0}$ is much smaller in magnitude than $u_{s(o)}$ : there is then an abrupt change in $u_{s}$ near the end of the film across the boundary layer. The transport of surfactant from the Plateau border to film, which relies on transport at the velocity $\left.u_{s}\right|_{x=0}$, is much more limited than it would have been had the symmetry point on the Plateau border not been constraining the motion.

Our numerical results and asymptotic analyses (both to be presented later) indicate that when $a^{\prime} \ll a_{c r i t}^{\prime}$ (in our current notation, when $\Delta_{0} M_{s} \gg 1$ ) the decay of $u_{s}$ towards 
zero really is uniform along the entire surface of the border, exactly as equation (12) suggests. Indeed in this limit it turns out not even to be necessary to assume that the curved border is 'unfolded' along the lines discussed earlier. Equation (12) is then an excellent approximation for matching the film to the border.

Note that the study of Vitasari et al. (2015) not only considered the case $a_{c r i t}^{\prime} \gg a^{\prime}$, i.e. $\delta_{0}^{\prime} \bar{\mu}_{s} \gg a^{\prime 2}$, i.e. $\Delta_{0} M_{s} \gg 1$, but also considered another more 'extreme' case $\delta_{0}^{\prime} \bar{\mu}_{s} \gg 1$, i.e. $\Delta_{0} M_{s} \gg\left(a^{\prime}\right)^{-2}$. Given that $a^{\prime}<1$ (and in fact for a dry foam $a^{\prime} \ll 1$ ), this latter case is automatically covered by $\Delta_{0} M_{s} \gg 1$, and equation (12) (compatible with a uniform surface rate of strain on the Plateau border) continues to apply. In fact it turns out to be not equation (12) but rather equation (13) which breaks down in this particular limit, i.e. there is no longer a velocity boundary layer. As a consequence, the derivation of equation (16) ceases to be valid. This situation has however been explored already by Vitasari et al. (2015), and therefore we do not need to give the case $\Delta_{0} M_{s} \gg\left(a^{\prime}\right)^{-2}$ any further special consideration here.

We wish to consider instead what happens when one hypothesises a non-uniform surface rate of strain on the Plateau border surface. This is achieved in the next subsection.

\subsection{Non-uniform strain rate hypothesis}

As stated above, we want to consider the case of non-uniform strain rates on the Plateau border surface. In particular we want to know what happens if the magnitude of the strain rate is biggest near the entrance to the Plateau border (where it joins up with the film) such that $u_{s}$ decays to zero or near zero over a distance that is much smaller than the distance $\pi a / 6$ (or equivalently $L \frac{\pi}{6} a^{\prime}$ ) between the border entrance and its central symmetry point. Such a rapid velocity decay is constraining the Plateau border flow, which in turn constrains the film flow near the border. The ratio between the length scale of the velocity boundary layer on the film $L \frac{\pi}{6} a_{\text {crit }}^{\prime}$ and the decay length on the Plateau border is now rather larger than $a_{c r i t}^{\prime} / a^{\prime}$ which was the estimated value of this ratio originally used in equation (16). As a result, the actual value of $\left.u_{s}\right|_{x=0}$ is rather smaller than equation (16) would predict. In what follows we shall suppose that, even when $a_{\text {crit }}^{\prime} \ll a^{\prime}$, the velocity decay length on the Plateau border can be selected 
to be comparable with the length scale of the velocity boundary layer on the film. The rationale for selecting this particular length scale as being relevant to velocity decay on the Plateau border will be discussed later. For the present though, we wish to explore the consequences of selecting it. The effect is to make the film aware of the constraints from the Plateau border, but nevertheless still unaware of the actual size of the Plateau border (since this is substantially larger than the velocity decay length).

In lieu of equation (12), we assume a formula for $\partial u_{s} /\left.\partial x\right|_{x=0}$ that is compatible with the above mentioned decay length scale, i.e.

$$
\begin{aligned}
\partial u_{s} /\left.\partial x\right|_{x=0} & =-\left.c u_{s}\right|_{x=0} /\left(L \sqrt{\delta_{0}^{\prime} \bar{\mu}_{s}}\right) \equiv-\left.c u_{s}\right|_{x=0} /\left(L \frac{\pi \sqrt{3}}{6} a_{c r i t}^{\prime}\right) \\
& \equiv-\left.c u_{s}\right|_{x=0} /\left(a \sqrt{\Delta_{0} M_{s}}\right)
\end{aligned}
$$

where $c$ is an unknown value (ideally of order unity, but yet to be determined).

In that case (even if $a^{\prime} \gg a_{c r i t}^{\prime}$, i.e. even if $\Delta_{0} M_{s} \ll 1$ ), the magnitude of $\left.u_{s}\right|_{x=0}$ (the velocity at the end of the film) will always be significantly less than that of $u_{s(o)}$ (the velocity immediately outside the boundary layer). In fact (combining equation (17) with equation (13)) implies

$$
\left.u_{s}\right|_{x=0} \approx \frac{\sqrt{3}}{(\sqrt{3}+c)} u_{s(o)}
$$

an important equation to which we will return later.

Note that equation (17) only implies (18) when equation (13) is also valid. We have already stated that (13) might lose validity when the $\gamma$ vs $x$ field on the film develops a boundary layer character (instead of just $u_{s}$ vs $x$ exhibiting boundary layers). In such situations (17) can still be valid, even though (18) is not. We will not study such situations in any detail in what follows, although we will return to this point much later on.

In summary, what we are now claiming is that if $a^{\prime} \ll a_{\text {crit }}^{\prime}$ (equivalently $\Delta_{0} M_{s} \gg 1$ ), then equation (12) still applies and consequently $\left.u_{s}\right|_{x=0}$ is much smaller in magnitude than $u_{s(o)}$ as equation (16) then suggests. On the other hand, if $a^{\prime} \gg a_{c r i t}^{\prime}$ (i.e. $\Delta_{0} M_{s} \ll 1$ ), equation (12) does not apply, but should be replaced by equation (17) instead. As a result, instead of equation (16) (which would now erroneously predict $\left.u_{s}\right|_{x=0}$ very close to 
$\left.u_{s(o)}\right)$, we need to employ equation (18), which predicts somewhat smaller $\left.u_{s}\right|_{x=0}$ values.

\section{Dimensionless groups and their magnitude}

In the foregoing discussion we identified a number of key dimensionless groups. For analysing the Plateau border flow, these were $\Delta_{0} \equiv \delta / a$ (dimensionless film thickness) and $M_{s}=\mu_{s} /(\mu a)$ (dimensionless surface viscosity). For analysing the film flow, we identified instead $\delta_{0}^{\prime} \equiv \delta_{0} / L$ (film aspect ratio), $\bar{\mu}_{s}=\mu_{s} /(\mu L)$ (again a dimensionless surface viscosity, but scaled differently from $M_{s}$ ), $a^{\prime}=a / L$ (dimensionless curvature radius of the Plateau border), and $a_{c r i t}^{\prime}$ (defined in terms of $\delta_{0}^{\prime}$ and $\bar{\mu}_{s}$ in equation (14)). Not all these dimensionless groups are independent of one another, some being more relevant to the Plateau border, and others more relevant to the film, but when trying to match the Plateau border flow to the film flow, it is useful to consider them all.

In what follows estimates of the values of these dimensionless groups are given (Section 5.1) and then the governing equations developed earlier in Sections 2-4 are cast in dimensionless form involving the relevant dimensionless groups (Section 5.2).

\subsection{Typical values of dimensionless groups}

Estimates of the above dimensionless groups have been obtained (Vitasari et al., 2015) under conditions typical of a fractionation process.

The geometric factor $\delta_{0}^{\prime}$ (film thickness to film length) was estimated to be small: around $4 \times 10^{-3}$ was typical for a freshly formed film, but a smaller value $3 \times 10^{-6}$ was typical of a film that has already had an opportunity to drain.

The geometric factor $a^{\prime}$ was estimated (Vitasari et al., 2015) to be 0.1 typically. This can be shown to correspond to a fairly dry foam (Vitasari, 2014) (liquid fraction $0.17 \%$ assuming a Kelvin cell bubble structure). A wetter foam would have a larger $a^{\prime}$ value: $a^{\prime}$ is known to be proportional to the square root of liquid fraction (Vitasari, 2014), so significant changes in liquid fraction imply less significant changes in $a^{\prime}$.

The value of $\Delta_{0}$ (which happens to equal $\delta_{0}^{\prime} / a^{\prime}$ ) is an order of magnitude larger than that of $\delta_{0}^{\prime}$ (somewhere in the range $4 \times 10^{-2}$ down to $3 \times 10^{-5}$ being typical). It is clear that $\Delta_{0}$ (like $\delta_{0}^{\prime}$ itself) is a parameter that is significantly smaller than unity. 
Considerable uncertainty surrounds the 'typical' value of the dimensionless rheological parameter $\bar{\mu}_{s}$. This is partly because different surface active materials will have very different surface viscosities, so $\bar{\mu}_{s}$ is strongly material dependent. However the uncertainty is also partly because (even for a given surface active material) different measurement techniques can lead to wildly different values (Stevenson, 2005; Vitasari et al., 2015).

The surface active protein bovine serum albumin (BSA) is a material that has been used in fractionation studies previously (Brown et al., 1990). For a typical fractionation set up, the value of $\bar{\mu}_{s}$ was estimated (Vitasari et al., 2015) to be 880, using a surface viscosity value determined by Durand and Stone (2006). The common surfactant sodium dodecyl sulphate (SDS) was found by Durand and Stone (2006) to have a surface viscosity two orders of magnitude smaller than BSA. Different measurement techniques however find much smaller surface viscosities for SDS (smaller by three or more orders of magnitude): see Vitasari et al. (2015) and references therein. Thus, with $a^{\prime}=0.1$ as above, the parameter $M_{s}$ (which equals $\bar{\mu}_{s} / a^{\prime}$ ) could be as large as 8800 for BSA, but could be five (or more) orders of magnitude smaller for a different material (SDS) and/or if a different measurement technique is deemed more reliable than that of Durand and Stone (2006).

The computations to follow will employ $a^{\prime}$ fixed at 0.1 , but with $\Delta_{0}$ and $M_{s}$ values covering the wide ranges identified above. Before proceeding with detailed calculations however, it is convenient to cast our governing equations in dimensionless form.

\subsection{Governing equations in dimensionless form}

We choose different dimensionless scales in the governing equations according to whether we treat flow on the film (Section 5.2.1) or Plateau border (Sections 5.2.2-5.2.3).

\subsubsection{Dimensionless governing equations for the film}

For the film we make distances dimensionless with respect to the film half-length $L$. The dimensionless coordinate $x^{\prime}$ runs from the centre of the film (at $x^{\prime}=-1$ ) to the junction with the Plateau border $\left(\right.$ at $\left.x^{\prime}=0\right)$.

We make surface tensions $\gamma$ dimensionless with respect to a Gibbs parameter ${ }^{4} G$.

\footnotetext{
${ }^{4}$ We define $G$ as a Gibbs elasticity, the absolute magnitude of the derivative of $\gamma$ with respect to the
} 
This parameter $G$ governs variations of surface tension on the film: if there are significant relative changes in the surfactant coverage along the film, we expect variations in $\gamma$ up to the order of $G$. For the systems treated here (Vitasari et al., 2015, 2013b), $G$ is comparable in size to $\gamma_{0}$, the constant tension at the point where the film and border join.

Velocities on the film are made dimensionless on the scale $G \delta_{0}^{\prime} / \mu$, a scale which arises from balancing Marangoni stresses with viscous shear stress in the bulk of the film.

Equation (4) becomes

$$
3 u_{s}^{\prime}=\partial \gamma^{\prime} / \partial x^{\prime}+\delta_{0}^{\prime} \bar{\mu}_{s} \partial^{2} u_{s}^{\prime} / \partial x^{\prime 2}
$$

where $u_{s}^{\prime}$ is dimensionless velocity and $\gamma^{\prime}$ is dimensionless surface tension.

The dimensionless analogues of equations (12) and (16) are

$$
\begin{gathered}
\partial u_{s}^{\prime} /\left.\partial x^{\prime}\right|_{x^{\prime}=0}=-\left.u_{s}^{\prime}\right|_{x^{\prime}=0} /\left(\pi a^{\prime} / 6\right) \\
\left.u_{s}^{\prime}\right|_{x^{\prime}=0} \approx u_{s(o)}^{\prime}\left(1+a_{c r i t}^{\prime} / a^{\prime}\right)^{-1}=u_{s(o)}^{\prime}\left(1+\frac{6}{\pi} \sqrt{\frac{\Delta_{0} M_{s}}{3}}\right)^{-1},
\end{gathered}
$$

$u_{s(o)}^{\prime}$ being dimensionless velocity outside a boundary layer. Analogues of (17)-(18) are

$$
\begin{gathered}
\partial u_{s}^{\prime} /\left.\partial x^{\prime}\right|_{x^{\prime}=0}=-\left.c u_{s}^{\prime}\right|_{x^{\prime}=0} / \sqrt{\delta_{0}^{\prime} \bar{\mu}_{s}} \equiv-\left.c u_{s}^{\prime}\right|_{x^{\prime}=0} /\left(a^{\prime} \sqrt{\Delta_{0} M_{s}}\right) \\
\equiv-\left.c u_{s}^{\prime}\right|_{x^{\prime}=0} /\left(\frac{\pi \sqrt{3}}{6} a_{c r i t}^{\prime}\right) \\
\left.u_{s}^{\prime}\right|_{x^{\prime}=0} \approx \frac{\sqrt{3}}{(\sqrt{3}+c)} u_{s(o)}^{\prime} .
\end{gathered}
$$

\subsubsection{Dimensionless governing equations for the Plateau border}

For the 2-d flow in the Plateau border we make distances dimensionless on the scale $a$, with $r^{\prime}$ denoting the dimensionless radial coordinate. The dimensionless arc length (denoted $S$ and measured along the Plateau border) is now identical to the angular coordinate $\theta$. The symbol $\bar{\nabla}$ denotes the dimensionless gradient operator. We also define two

\footnotetext{
logarithm of surfactant surface excess, evaluated for the particular surface excess on the Plateau border.
} 
sets of dimensionless Cartesian coordinates: an $X$ coordinate (measured along the Plateau border from the point where the border meets the film), and a $Y$ coordinate (measured across the border), as well as $\bar{x}, \bar{y}$ coordinates (rotated with respect to $X$ and $Y$, and with their origin outside the Plateau border at the point where $r^{\prime}=0$; see Figure 2).

On the film (see Section 5.2.1) velocities were made dimensionless on a 'Marangoni' velocity scale $G \delta_{0}^{\prime} / \mu$. Since we assume no Marangoni stresses on the Plateau border surface, we select a different velocity scale there: velocities are made dimensionless on the scale $\left.|u|_{r=a, \theta=0}|\equiv| u_{s}\right|_{S=0} \mid$, the absolute value recognizing that $\left.u_{s}\right|_{S=0}$ is typically negative.

Dimensionless velocity $\boldsymbol{U}$ has components either $U_{r}$ and $U_{\theta}$ (in polar coordinates) or $U_{X}$ and $U_{Y}$ (in Cartesian coordinates), while the velocity along the Plateau border surface is $U_{s}$. Based on the way we non-dimensionalise velocity, we deduce $\left.U_{s}\right|_{S=0}=-1$. Pressure meanwhile is non-dimensionalised on the scale $\mu\left|u_{s}\right|_{S=0} \mid / a$ (equivalently on the scale $\left.\left.G \delta_{0}^{\prime}\left|u_{s}^{\prime}\right|_{S=0}\left|/ a \equiv G L^{-1} \Delta_{0}\right| u_{s}^{\prime}\right|_{S=0} \mid\right)$, and we denote the dimensionless pressure by $p$.

Continuity implies $\bar{\nabla} \cdot \boldsymbol{U}=0$. The dimensionless Stokes flow equation is

$$
0=-\bar{\nabla} p+\bar{\nabla}^{2} \boldsymbol{U}
$$

On the Plateau border surface $\left(r^{\prime}=1\right)$, boundary conditions are $U_{r}=0$ and also

$$
-r^{\prime} \frac{\partial}{\partial r^{\prime}}\left(\frac{U_{\theta}}{r^{\prime}}\right)=M_{s} \frac{\partial^{2} U_{s}}{\partial S^{2}}
$$

At the entrance to the Plateau border $(\theta=0)$ we have a boundary condition

$$
U_{\theta}=-\frac{3}{2} \frac{\left(1+\Delta_{0}-r^{\prime}\right)^{2}}{\Delta_{0}^{2}}+\frac{1}{2}
$$

and we also set the dimensionless shear stress to the known function $\partial U_{\theta} / \partial r^{\prime}$. Symmetry conditions on $\theta=\frac{\pi}{6}$ and also on $r^{\prime}=\left(1+\Delta_{0}\right) / \cos \theta$ are $U_{n}=0$ and $\boldsymbol{n} . \bar{\nabla} U_{t}=0$ where $U_{n}$ and $U_{t}$ are normal and tangential velocity components and $\boldsymbol{n}$ denotes a normal vector.

In addition to this, we are free to set a zero for the pressure scale. One possible way of setting this is to assume zero pressure in the gas phase outside the Plateau border, which 
(owing to the curvature of the Plateau border surface) should give a negative pressure in the liquid in the Plateau border. However in view of the fact that we have imposed a condition of uniform curvature on the Plateau border surface, rather than imposing a normal stress condition along it, we can only set zero pressure in the gas phase at one particular point on the Plateau border surface, which we take to be the symmetry point $\theta=\frac{\pi}{6}$. We then estimate the (dimensionless) pressure in the liquid at this point to be $-\left(\Delta_{0} a^{\prime}\right)^{-1}$. The magnitude of this has been obtained by dividing the dimensional YoungLaplace pressure $\gamma_{0} / a$ through by the scale $G L^{-1} \Delta_{0}\left|u_{s}^{\prime}\right|_{S=0} \mid$. Defining $\gamma_{0}^{\prime}=\gamma_{0} / G$ and $a^{\prime}=a / L$, this evaluates to $\gamma_{0}^{\prime}\left(\Delta_{0} a^{\prime}\left|u_{s}^{\prime}\right|_{S=0} \mid\right)^{-1}$. In the case of the protein BSA it has been found (Vitasari et al., 2015) that $\gamma_{0}^{\prime}$ is order unity (based on data sourced from Durand and Stone (2006)). Meanwhile the dimensionless velocity $\left|u_{s}^{\prime}\right|_{S=0} \mid$ at the film-Plateau border junction is sensitive to how surfactant is distributed along the film, but should be no larger than order unity, based on the scalings employed for the film flow ${ }^{5}$. Hence we estimate $\gamma_{0}^{\prime}\left(\Delta_{0} a^{\prime}\left|u_{s}^{\prime}\right|_{S=0} \mid\right)^{-1}$ to be $\left(\Delta_{0} a^{\prime}\right)^{-1}$ and set this to be (the absolute magnitude of) the liquid pressure on the Plateau border surface at $\theta=\frac{\pi}{6}$. The pressure that we compute at other $\theta$ values along the Plateau border surface will differ from $-\left(\Delta_{0} a^{\prime}\right)^{-1}$. However as long as the variation in pressure relative to $\left(\Delta_{0} a^{\prime}\right)^{-1}$ is small, our approximation that the Plateau border surface has a uniform curvature remains valid.

\subsubsection{Dimensionless quasi-1-d equations for the Plateau border}

In addition to the 2-d formulation described above, we can also obtain a dimensionless version of the quasi-1-d Plateau border flow (from Section 3.5) applicable for dimensionless $X$ values considerably smaller than unity. The velocity component $U_{Y}$ is much smaller than $U_{X}$, and so the flow is dominated by the latter which we denote simply by $U$.

The governing equations are as follows. In dimensionless form, equation (9) becomes

$$
U \approx U_{s}(X)\left(\frac{3}{2} \frac{Y^{2}}{(\Delta(X))^{2}}-\frac{1}{2}\right)
$$

\footnotetext{
${ }^{5}$ Note that $\left|U_{s}\right|_{S=0} \mid$ is unity by definition, but $\left|u_{s}^{\prime}\right|_{S=0} \mid$ is scaled differently and differs from unity.
} 
with dimensionless border thickness

$$
\Delta \approx \Delta_{0}+X^{2} / 2
$$

Equation (11) (neglecting Marangoni effects in the Plateau border) becomes

$$
\partial U /\left.\partial Y\right|_{Y=\Delta}=M_{s} \partial^{2} U_{s} / \partial X^{2}
$$

from which it follows

$$
3 U_{s} / \Delta=3 U_{s} /\left(\Delta_{0}+X^{2} / 2\right)=M_{s} \partial^{2} U_{s} / \partial X^{2}
$$

where recall $\Delta_{0}$ is a small parameter. This is the key equation which we must solve.

We seek the value of $\partial U_{s} /\left.\partial X\right|_{X=0}$ (or equivalently $\partial U_{s} /\left.\partial S\right|_{S=0}$ since Cartesian coordinate $X$ and arc length coordinate $S$ coincide at $X=S=0$ ). These values can be used to couple the film flow $u_{s}^{\prime}$ and Plateau border flow $U_{s}$. Equation (20) hypothesises that

$$
a^{\prime}\left|u_{s}^{\prime}\right|^{-1} \partial u_{s}^{\prime} /\left.\partial x^{\prime}\right|_{x^{\prime}=0} \equiv \partial U_{s} /\left.\partial S\right|_{S=0} \approx 6 / \pi
$$

whereas equation (22) gives (for a value of $c$ to be determined)

$$
a^{\prime}\left|u_{s}^{\prime}\right|^{-1} \partial u_{s}^{\prime} /\left.\partial x^{\prime}\right|_{x^{\prime}=0} \equiv \partial U_{s} /\left.\partial S\right|_{S=0}=c / \sqrt{\Delta_{0} M_{s}}
$$

In the above (for both 2-d and quasi-1-d systems) there is an assumption (to be checked a posteriori) that $U_{s}$ and hence $\partial U_{s} / \partial X$ (or equivalently $\partial U_{s} / \partial S$ ) vary on length scales large compared to $\Delta_{0}^{1 / 2}$. The reason is that Marangoni stresses are neglected in equations (25), (29)-(30) assuming that Plateau borders act as surfactant reservoirs due to being much thicker than films, which can only be true for $X$ larger than order $\Delta_{0}^{1 / 2}$. 


\section{Case permitting analytic solution for the velocity field in the film}

In the first instance we consider the velocity field on the film. Either equation (20) or (22) allows us to close the set of equations determining this. All the above discussion has been generic without assuming any particular field for surface tension $\gamma^{\prime}$ vs $x^{\prime}$ along the film. In order to calculate actual velocity fields $u_{s}^{\prime}$ vs $x^{\prime}$ in this system, it is necessary to know the value of $\gamma^{\prime}$ vs $x^{\prime}$ (which ultimately depends on the instantaneous distribution of surfactant, that in turn is also coupled to the velocity field via the surfactant mass balance): for the sake of illustration, a simple and easy-to-solve case with a specified surfactant distribution is considered in what follows. The $u_{s}^{\prime}$ vs $x^{\prime}$ velocity fields that we compute of course drive the subsequent time evolution $\gamma^{\prime}$ vs $x^{\prime}$ (although we do not consider this aspect here, focussing just on the instantaneous velocity field $u_{s}^{\prime}$ ). In the easyto-solve case that we consider here, the contrast between the consequences of equation (12) and those of (17) becomes readily apparent.

We will suppose for convenience that

$$
\gamma^{\prime}-\gamma_{0}^{\prime}=\frac{\beta}{2}\left(1-\left(x^{\prime}+1\right)^{2}\right)
$$

$\gamma_{0}^{\prime}$ is the (assumed constant and uniform) surface tension on the Plateau border (nondimensionalised here with respect to the Gibbs parameter $G$ ) and $\beta$ is a dimensionless constant ${ }^{6}$. This is a simple but plausible distribution of surface tension on a film during the foam fractionation scenarios of interest here: film surface tension is on average higher than that on the Plateau borders, and moreover local surface tension grows as one moves towards the centre of the films, away from the Plateau borders.

We are interested in cases where the parameter $a_{\text {crit }}^{\prime}$ is no larger than order $a^{\prime}$, since only in such cases is there any ambiguity regarding which boundary condition to use (assumption (20) vs assumption (22)). Under these circumstances the velocity field admits a 'boundary layer', a decaying exponential which decays rapidly as $x^{\prime}$ moves into the

\footnotetext{
${ }^{6}$ In the systems of interest, the value of $\beta$ depends on the relative differences in surfactant concentration between surfactant-rich material in Plateau borders, and surfactant-lean material in films. Assuming a significant relative concentration difference, we can treat $\beta$ as being a constant of order unity.
} 
film away from the Plateau border. It follows moreover that the boundary layer length ( $\sqrt{\delta_{0}^{\prime} \bar{\mu}_{s} / 3}$ in dimensionless units) is no larger than order $a^{\prime}$ (with dimensionless Plateau border curvature radius $a^{\prime}$ itself being much smaller than unity).

The solution to equation (19) for $u_{s}^{\prime}$ that satisfies condition (20) is

$$
\begin{aligned}
u_{s}^{\prime} & =-\frac{\beta\left(x^{\prime}+1\right)}{3}+\frac{\beta\left(1+a^{\prime} \pi / 6\right)}{3\left(1+a^{\prime} / a_{c r i t}^{\prime}\right)} \exp \left(\frac{x^{\prime}}{\sqrt{\delta_{0}^{\prime} \bar{\mu}_{s} / 3}}\right) \\
& \equiv-\frac{\beta\left(x^{\prime}+1\right)}{3}+\frac{\beta\left(1+a^{\prime} \pi / 6\right)}{3\left(1+a^{\prime} / a_{\text {crit }}^{\prime}\right)} \exp \left(\frac{x^{\prime}}{\frac{\pi}{6} a_{c r i t}^{\prime}}\right) .
\end{aligned}
$$

Hence at the end of the film where it joins the Plateau border (i.e. at $x^{\prime}=0$ )

$$
\left.u_{s}^{\prime}\right|_{x^{\prime}=0}=-\frac{\beta}{3} \frac{\left(\left(a^{\prime} / a_{c r i t}^{\prime}\right)-\frac{\pi}{6} a^{\prime}\right)}{\left(1+a^{\prime} / a_{c r i t}^{\prime}\right)}
$$

which is actually consistent with equation (21). Remember that $a_{c r i t}^{\prime}$ is no larger than order $a^{\prime}$ here, so the right hand side of equation (35) is reasonably close to $-\beta / 3$. Indeed we can denote this value $-\beta / 3$ by $u_{s(o)}^{\prime}$ the dimensionless velocity immediately outside the exponential 'boundary layer'. Both velocities $\left.u_{s}^{\prime}\right|_{x^{\prime}=0}$ and $u_{s(o)}^{\prime}$ are of course negative (i.e. in the direction from Plateau border to film) since in the systems of interest, Marangoni stresses drive motion from surfactant-rich Plateau borders to surfactant-lean films, as well as along the surfaces of the films, towards their centres.

An illustrative case is plotted in Figure 3 using dimensionless parameter values ${ }^{7} \bar{\mu}_{s}=$ 0.088 and $\delta_{0}^{\prime}=4 \times 10^{-3}$ (with $\sqrt{\delta_{0}^{\prime} \bar{\mu}_{s} / 3} \approx 0.010$ and hence $a_{\text {crit }}^{\prime}=\frac{6}{\pi} \sqrt{\delta_{0}^{\prime} \bar{\mu}_{s} / 3} \approx 0.020$ ) and also with $a^{\prime}=0.1$. The dimensionless parameter $\beta$ is taken arbitrarily to equal unity.

In the case of assumption (22), the solution for the velocity field becomes instead

$$
u_{s}^{\prime}=-\frac{\beta\left(x^{\prime}+1\right)}{3}+\frac{\beta}{3} \frac{\left(\frac{c}{\sqrt{3}}+\frac{\pi}{6} a_{c r i t}^{\prime}\right)}{(1+c / \sqrt{3})} \exp \left(\frac{x^{\prime}}{\sqrt{\delta_{0}^{\prime} \bar{\mu}_{s} / 3}}\right) .
$$

\footnotetext{
${ }^{7}$ The choice of $\bar{\mu}_{s}=0.088$ for this illustrative case recognizes from Section 5.1 that the parameter $M_{s}$ (and hence the parameter $\bar{\mu}_{s} \equiv M_{s} a^{\prime}$ with $a^{\prime}=0.1$ here) can be orders of magnitude smaller than the 'nominal' $M_{s}$ value of 8800 quoted in that section.
} 
From equation (36) we deduce at the end of the film

$$
\left.u_{s}^{\prime}\right|_{x^{\prime}=0}=-\frac{\beta}{3} \frac{\left(1-\pi a_{c r i t}^{\prime} / 6\right)}{(1+c / \sqrt{3})}
$$

which is consistent with equation (23), and (given that $c$ is expected to be of order unity) suggestive of a velocity with a magnitude rather smaller than $\beta / 3$.

Figure 3 plots the velocity profile (36) assuming $c=\sqrt{3}$ (chosen arbitrarily for the moment, albeit to be justified later on) and all other parameter values as before. We can see that under these circumstances, the velocity at the end of the film $\left.u_{s}^{\prime}\right|_{x^{\prime}=0}$ is more constrained than with equation (34).

Corroborating the discussion of Sections 4.2-4.3, a comparison between equations (37) and (35) tells us how much less $u_{s}^{\prime}$ at the end of the film is in the case of boundary condition (22) compared to (20). We can however only effect this comparison if we are able to determine the value of the parameter $c$. This requires detailed knowledge of the flow field in the Plateau border and is the topic of the sections to follow.

\section{Two-dimensional fluid dynamical calculations}

The 2-d Stokes flow equations for the Plateau border described in Section 5.2.2 have been implemented in the finite element software COMSOL multiphysics using polar $r^{\prime}, \theta$ coordinates. Although it is possible to solve numerically the coupled Stokes flow equations for the Plateau border and film together, in practice we avoid this, as the film would need to be discretised into very tiny elements due to being much thinner than the Plateau border. It is less expensive to exploit lubrication type assumptions in the film (as per Section 2) and perform COMSOL finite element calculations solely for the Plateau border.

We encountered a slight difficulty with implementing the symmetry conditions $\left(U_{n}=0\right.$ and $\left.\boldsymbol{n} \cdot \bar{\nabla} U_{t}=0\right)$ at the boundary $r^{\prime}=\left(1+\Delta_{0}\right) / \cos \theta$, which is a straight line in Cartesian coordinates, but curved in polar coordinates. COMSOL did not permit us to represent the boundary of our solution domain via an arbitrary $r^{\prime}$ vs $\theta$ curve. It did however permit us to divide the boundary up into a large number of segments (1000 segments) with $r^{\prime}$ vs $\theta$ on each segment being represented by a Bezier curve. The Bezier curves were chosen 
such that for each segmented interval of $\theta$, the values of $r^{\prime}$ and $\mathrm{d} r^{\prime} / \mathrm{d} \theta$ at the start and end of the interval matched the true boundary $r^{\prime}=\left(1+\Delta_{0}\right) / \cos \theta$. The conditions $U_{n}=0$ and $\boldsymbol{n} \cdot \bar{\nabla} U_{t}=0$ were then applied to these Bezier representations of the symmetry line (despite the fact that the Bezier representation does not quite coincide with the original symmetry line, and hence symmetry should be very slightly broken along it).

There was invariably some noise detectable in our simulation data on the length scale of the Bezier intervals, but it was tiny compared to the velocities calculated. Moreover the use of Bezier curves in the polar coordinate representation of the symmetry boundary proved far less noisy than using piecewise linear (or 'straight') $r^{\prime}$ vs $\theta$ segments in that polar coordinate representation, which would imply $\mathrm{d} r^{\prime} / \mathrm{d} \theta$ jumps from segment to segment.

\subsection{Numerical implementation}

We implemented a numerical 2-d simulation in COMSOL, for values of $\Delta_{0}$ equal to $5 \times 10^{-2}, 5 \times 10^{-3}, 5 \times 10^{-4}$, and $5 \times 10^{-5}$. The finite element mesh was chosen adaptively by COMSOL. Figure 4 shows a 'sample' mesh in the case $\Delta_{0}=0.05$. This has been converted from a mesh in polar $r^{\prime}, \theta$ coordinates to Cartesian $\bar{x}, \bar{y}$ coordinates (see Figure 2 for definitions of $\bar{x}$ and $\bar{y}$; compared to the $X, Y$ system, the origin has been shifted and the coordinate frame rotated). To aid clarity, the mesh shown has only 1558 elements, and the symmetry line $r^{\prime}=\left(1+\Delta_{0}\right) / \cos \theta$ (which maps to $\left.\bar{x}=1+\Delta_{0}\right)$ is constructed using only 100 Bezier segments in $r^{\prime}, \theta$ space (instead of the usual 1000 segments). The meshes we actually used in our computations were significantly denser (see below). Unsurprisingly Figure 4 shows smaller elements being chosen near the entrance to the Plateau border near $\theta=0$ (owing to the thinner geometry there) but elements could be rather larger near $\theta=\pi / 6$ where the border has opened much wider. Smaller elements are also seen along $\bar{x}=1+\Delta_{0}$ (as a consequence of the aforementioned Bezier representation).

As stated above, the meshes used in our computations were denser than the one in Figure 4. Even with these dense meshes however, there was a marked tendency towards having fewer elements in more 'extreme' geometries as $\Delta_{0}$ was decreased. This was partly due to the fact that, as $\Delta_{0}$ decreased, the overall size of the solution domain decreased slightly. However it was also due to the fact that, as $\Delta_{0}$ decreased, significant velocities 
tended to be confined closer and closer to the entrance to the Plateau border, meaning rather large elements could be used elsewhere in the domain. For instance 191503 elements (869680 degrees of freedom) were used when $\Delta_{0}=5 \times 10^{-2}$ but only 92155 elements (435612 degrees of freedom) were used for $\Delta_{0}=5 \times 10^{-5}$. Simulations with $\Delta_{0}=5 \times 10^{-2}$ took approximately 40 seconds on a PC with an Intel Core i5 and 6Gb RAM memory, with those for smaller $\Delta_{0}$ values running slightly more quickly ${ }^{8}$. Notice however that the smallest $\Delta_{0}$ values also are amenable to asymptotic approaches (see Section 5.2.3).

We studied values of dimensionless surface viscosity $M_{s}$ over a wide range. We considered $^{9} M_{s}$ values in powers of ten from $10^{4}$ down to $10^{-6}$, and also considered $M_{s}=0$.

Results of the COMSOL simulations are presented and discussed in the next section.

\section{Numerical results}

This section is laid out as follows. In Section 8.1 we show computed streamline patterns in the Plateau border. Then in Section 8.2 we show velocity profiles along the gas-liquid Plateau border surface. Next in Section 8.3 we analyse the surface strain rate at the filmPlateau border junction, which is essential for coupling the film and Plateau border flows together. Next in Section 8.4 we consider pressure distributions. Finally in Section 8.5 we discuss the impact of surface viscous effects on surfactant mass transfer rates.

\subsection{Streamline patterns}

In what follows we consider streamline patterns with two distinct $\Delta_{0}$ values: $\Delta_{0}=0.05$ (Section 8.1.1) and $\Delta_{0}=0.005$ (Section 8.1.2). Topologies of the streamline patterns are discussed in Section 8.1.3. Finally Section 8.1.4 places a special focus on a particular $M_{s}$ value, namely $M_{s}=1$.

\footnotetext{
${ }^{8}$ Note that the 40 second run time reported here gives just the velocity field in the Plateau border arising due to the surfactant distribution on the film for a given instant in time. The run times reported in the introduction to the paper corresponding to the simple model of Vitasari et al. (2015) (e.g. just a few minutes run time with surface viscosity) considered the entire evolution of the surfactant concentration field, with on the order of $10^{4}$ time steps. This corresponds to a mere 0.02 s run time per step.

${ }^{9}$ In addition to the various cases described here, one can solve a formal limit of $M_{s} \rightarrow \infty$ for which one imposes a tangential velocity condition on the gas-liquid Plateau border surface $U_{s}=-(1-6 S / \pi)$ in lieu of equation (25). However the case $M_{s}=10^{4}$ produced a $U_{s}$ profile that was already so close to that tangential velocity condition, we never found it necessary to tackle the formal $M_{s} \rightarrow \infty$ case.
} 


\subsubsection{Streamline patterns for $\Delta_{0}=0.05$}

Figure 5 shows three streamline patterns computed for $M_{s}=10^{4}, M_{s}=1$ and $M_{s}=$ 0.1 respectively all for the case $\Delta_{0}=0.05$. These streamline patterns have been computed in $r^{\prime}, \theta$ coordinates but plotted in terms of Cartesian $\bar{x}, \bar{y}$ variables as defined in Figure 2.

On each figure a total of 15 streamlines are shown (including the streamline on the domain boundary). It is clear that the streamline pattern for $M_{s}=10^{4}$ fills the entire domain, which seems consistent with a hypothesis of uniform decay of the surface velocity between the entrance to the Plateau border and the symmetry point on the Plateau border surface. This in fact follows directly from boundary condition (25). If $M_{s}$ is exceedingly large, $\partial^{2} U_{s} / \partial S^{2}$ must be small to compensate. Hence $\partial U_{s} / \partial S$ must be near uniform, and $U_{s}$ must be close to a straight line function of $S$

$$
U_{s} \approx-(1-6 S / \pi)
$$

which satisfies the correct boundary conditions $\left.U_{s}\right|_{S=0}=-1$ and $\left.U_{s}\right|_{S=\pi / 6}=0$ respectively at the Plateau border entrance and at the symmetry point on the Plateau border.

The streamline pattern for $M_{s}=1$ fills most of (but not quite all of) the domain: the spatial decay of the streamline pattern is noticeably faster than for $M_{s}=10^{4}$. Finally for $M_{s}=0.1$ the spatial decay of the streamline pattern is seen to be very abrupt indeed. This is definitely inconsistent with an assumption of uniform spatial decay of the velocity field along the entire Plateau border surface.

\subsubsection{Streamline patterns for $\Delta_{0}=0.005$}

Analogous data but with a smaller $\Delta_{0}$ (i.e. $\Delta_{0}=0.005$ ) are shown in Figure 6 . Each plot again has 15 streamlines, but (owing to the extremely thin geometry at the entrance to the Plateau border) it is not easy to see them all. This is particularly true for $M_{s}=0.1$ where the streamlines are all confined very near the entrance to the Plateau border, the rest of the Plateau border barely having any flow.

By contrast for $M_{s}=10^{4}$ it is still the case that the streamlines fill the entire Plateau border (as was also seen in Figure 5) but the streamline pattern is subtly different from 
what was seen before. Specifically the topology of the streamline pattern is different, as Figure 7 shows schematically: two internal stagnation points (one centre and one saddle) are present in the case with small $\Delta_{0}=0.005$ and $M_{s}=10^{4}$, and, associated with these stagnation points, there is a set of closed recirculation streamlines. In fact we observed this same topology in all cases studied with both $\Delta_{0} \leq 0.005$ and $M_{s} \geq 10$.

The explanation for this change in streamline topology is given in the next section.

\subsubsection{Streamline topology}

When $\Delta_{0}$ is small but $M_{s}$ is large (i.e. rather larger than unity) we have already seen that significant tangential flow on the gas-liquid Plateau border surface extends sufficiently far along the Plateau border that the velocity is still significant at points where the local border thickness $\Delta$ greatly exceeds the thickness $\Delta_{0}$ at the film-Plateau border junction. To satisfy continuity, a tangential flow velocity also arises on the symmetry line $r^{\prime}=\left(1+\Delta_{0}\right) / \cos \theta$ which is of opposite sign but similar order of magnitude to the flow on the gas-liquid surface $r^{\prime}=1$.

In the region where $\Delta \gg \Delta_{0}$, the flow field in the interior of the Plateau border set up by those tangential boundary flows, should not be too sensitive to precisely which boundary condition we impose at the film-Plateau border junction at $\theta=0$ between $r^{\prime}=1$ and $r^{\prime}=1+\Delta_{0}$, given that the length of that boundary is tiny. In particular if we were to change the boundary condition at $\theta=0$ so as to 'close off' the film-Plateau border junction and thereby not permit any fluid penetration whatsoever across any part of it, we would not expect to affect greatly the flow far from the film-Plateau border junction, provided we kept the tangential flows on $r^{\prime}=1$ and $r^{\prime}=\left(1+\Delta_{0}\right) / \cos \theta$ unchanged.

This 'closed off' variant of the flow field necessarily has closed recirculation streamlines. Given the tangential motion imposed on some of the domain boundaries, the resulting flow is reminiscent of a 'lid-driven' system (Ramanan and Homsy, 1994) for which a closed recirculation streamline topology might be anticipated. Returning to the Plateau border flow, since the 'true' flow field away from the neighbourhood of $\theta=0$ should be similar to the 'closed off' variant, it too must have closed recirculation streamlines.

The above argument does not however apply if $\Delta_{0}$ is increased: the film-Plateau border 
junction is then rather more significant, and a change to the boundary condition on that junction (from the original boundary to a 'closed off' one) would be rather more than just a weak perturbation to the flow. Thus there is no need to expect the same streamline topology in these two distinct cases.

Likewise the argument does not apply when $M_{s}$ is comparatively small (e.g. $M_{s}$ rather less than unity). In such cases the Plateau border flow field and specifically the tangential velocity on the gas-liquid Plateau border surface decays quite rapidly moving along the border. In the region where the tangential surface velocity is significant, the thickness of the Plateau border is still comparable with the thickness of the film. Thus the arguments suggesting a change in streamline topology towards a closed recirculation pattern do not apply here, because those arguments relied on flow managing to extend into regions where the Plateau border thickness vastly exceeded that of the film.

\subsubsection{Case $M_{s}=1$}

The above discussion in Section 8.1.2 considered $\Delta_{0}=0.005$ with $M_{s}=0.1$ and with $M_{s}=10^{4}$. Consider now the case $M_{s}=1$ in Figure $6(\mathrm{~b})$. As for the case $M_{s}=1$ with $\Delta_{0}=0.05$ (i.e. Figure $5(\mathrm{~b})$ ) this shows at least some streamlines penetrating along most of the solution domain. However for $\Delta_{0}=0.005$ relatively few streamlines (out of the 15 streamlines plotted) penetrate very far into the domain. This suggests a rapid velocity decay near the entrance to the Plateau border followed by a more gradual decay.

Understanding how a rapid decay followed by a gradual one might come about particularly for $M_{s}$ values near unity can be explained with reference to the quasi-1-d asymptotic model of Section 5.2.3. For sufficiently small $X$ values equation (30) simplifies to

$$
3 U_{s} / \Delta_{0} \approx M_{s} \partial^{2} U_{s} / \partial X^{2}
$$

The solution for $U_{s}$ is an exponential decay, decaying on a length scale $\sqrt{\Delta_{0} M_{s} / 3}$.

If $M_{s}$ is significantly smaller than unity then the exponential decay is complete long before $X$ becomes order $\Delta_{0}^{1 / 2}$ which is the regime for which equation (39) remains close to equation (30). It is however possible to query the physical basis for equation (30) (and 
hence equation (39)) under these circumstances: as alluded to in Section 5.2.3, Marangoni stresses in the Plateau border were neglected on the basis that it is much thicker than the film ${ }^{10}$ but that is not true for $X \leq O\left(\Delta_{0}^{1 / 2}\right)$.

If however $M_{s}$ is order unity or above, the exponential decay is 'frustrated'. As the decay of $U_{s}$ proceeds and $X$ increases, $\Delta$ becomes larger than $\Delta_{0}$, not only ensuring the physical validity of (30), but also making it deviate from (39). This increases the characteristic decay length further and further above $\sqrt{\Delta_{0} M_{s} / 3}$, so the decay slows down.

It is instructive to compare with Figure 5(c), which has $\Delta_{0}=0.05$ and $M_{s}=0.1$, giving the same value for the product $\Delta_{0} M_{s}$ as in Figure 6(b). Whereas Figure 5(c) shows a rapid decay of the velocity field (i.e. exponential decay with a characteristic decay length $\sqrt{\Delta_{0} M_{s} / 3}$, continuing of course to neglect any Marangoni stresses on the border), it is clear that Figure 6(b) exhibits a very different length scale for the overall decay, in view of the gradual decay component mentioned above.

We have also computed streamline patterns for yet smaller values of $\Delta_{0}$ i.e. $\Delta_{0}=$ 0.0005 and $\Delta_{0}=0.00005$, but the extremely thin geometry near where the Plateau border joins the film makes it difficult to resolve the patterns without a highly zoomed view in that part of the domain. In addition to the 2 -d streamline patterns however, it is also of interest to know the distribution of tangential velocity $U_{s}$ along the gas-liquid Plateau border surface. Such data are easy to plot even for exceedingly tiny $\Delta_{0}$ values, and are considered in the next section.

\section{2. $\left|U_{s}\right|$ vs $S$ curves}

In what follows we compare $\left|U_{s}\right|$ vs $S$ curves for two different $\Delta_{0}$ values $\Delta_{0}=0.005$ (Section 8.2.1) and $\Delta_{0}=0.00005$ (Section 8.2.2).

\subsection{1. $\left|U_{s}\right|$ vs $S$ for $\Delta_{0}=0.005$}

Curves for $\left|U_{s}\right|$ vs $S$ computed numerically are shown in Figure 8(a) for $\Delta_{0}=0.005$ and various $M_{s}$ (remember that by construction $\left.U_{s}\right|_{S=0}=-1$ so that $\left|U_{s}\right|_{S=0} \mid=1$ ).

\footnotetext{
${ }^{10}$ Strictly speaking we ignore Marangoni stresses on those parts of the border where it is thicker than the 'effective Henry constant' (see Section 3.5), the effective Henry constant being a physicochemical length scale bigger than the film thickness, but smaller than the curvature radius of the Plateau border.
} 
In Figure $8(\mathrm{a})$, for the case $\Delta_{0}=0.005$ and $M_{s}=10000$, we see $U_{s}$ is virtually a straight line function of $S$. The case $\Delta_{0}=0.005$ and $M_{s}=100$ also deviates comparatively little from a straight line. These data then correspond to the prediction of equation (38). The data match the assumption (31) used by Vitasari et al. (2015).

Looking instead at $\Delta_{0}=0.005$ and $M_{s}=0.1$ we see that $\left|U_{s}\right|$ vs $S$ is very far from a straight line function. Instead it follows very closely an exponential decay (with characteristic decay length $\sqrt{\Delta_{0} M_{s} / 3}$ as discussed in Section 8.1.4): the numerical data and the exponential decay curve are virtually indistinguishable on the scale of the graph.

The cases $\Delta_{0}=0.005$ and either $M_{s}=10$ or $M_{s}=1$ are intermediate between the situations described above. In particular $\Delta_{0}=0.005$ and $M_{s}=10$ shows a rapid initial decay which is arrested (at around $S=0.2$ with $\left|U_{s}\right|$ being roughly 0.4 at this point) to be followed by a straight line decay.

The case $\Delta_{0}=0.005$ and $M_{s}=1$ does not seem to attain a straight line regime as $S$ increases. Nevertheless the decay with increasing $S$ turns out to be rather slower than exponential (to avoid crowding the graph, we have not plotted the corresponding exponential $\exp \left(-S / \sqrt{\Delta_{0} M_{s} / 3}\right)$ for this particular $\Delta_{0}$ and $M_{s}$ combination). The numerical data for $\Delta_{0}=0.005$ and $M_{s}=1$ correspond then to the expected 'frustrated' exponential described in Section 8.1.4, i.e. the decay length grows even whilst the decay itself occurs.

\subsection{2. $\left|U_{s}\right|$ vs $S$ for $\Delta_{0}=0.00005$}

Data with the same $M_{s}$ values as before but for $\Delta_{0}=0.00005$ (not $\Delta_{0}=0.005$ ) are plotted in Figure 8(b).

We still see a straight line function for $M_{s}=10000$ and an exponential decay for $M_{s}=$ 0.1 (albeit for $\Delta_{0}=0.00005$ decaying on a much smaller length scale than previously).

The case $M_{s}=100$ now shows a moderate deviation from pure straight line behaviour, but with a straight line recovered for $S$ values greater than about 0.1 (see also further discussion of this case in the appendix). The case $M_{s}=10$ also shows a rapid initial decay which is subsequently arrested into a straight line decay. However the rapid initial decay progresses to rather smaller $\left|U_{s}\right|$ values when $\Delta_{0}=0.00005$ than when $\Delta_{0}=0.005$ (i.e. down to $\left|U_{s}\right|$ of roughly 0.2 , instead of roughly 0.4 ). Finally if we consider $M_{s}=1$ 
for $\Delta_{0}=0.00005$ we see that the decay of $\left|U_{s}\right|$ is substantially faster than was the case with $\Delta_{0}=0.005$ with that same $M_{s}$ value.

\subsubsection{Explaining the various decay modes for $U_{s}$}

In addition to $U_{s}$ exhibiting pure straight line decays (very large $M_{s}$ ) and pure exponential decays (values of $\Delta_{0}$ and $M_{s}$ both significantly smaller than unity), the data in Sections 8.2.1-8.2.2 show cases where a rapid initial decay is 'arrested' into straight line decay, and also other cases where a exponential decay is 'frustrated', becoming slower than exponential (although not necessarily a straight line decay).

We already explained in Section 8.1.4 via a quasi-1-d asymptotic analysis, how exponential decays for small $\Delta_{0}$ and $M_{s}$ values, become frustrated once $M_{s}$ values approach unity. We can also exploit the quasi-1-d asymptotic analysis to distinguish pure straight line decays from cases where rapid initial decays are arrested into straight line decays.

If $M_{s} \gg 1 / \Delta_{0}$, then it is clear (via equation (28)) that $M_{s} \gg 1 / \Delta$ for all $X$ values, and hence (via quasi-1-d equation (30)) that $\partial^{2} U_{s} / \partial X^{2}$ must be near zero for all $X$. This implies that $\partial U_{s} / \partial X$ must be near uniform for all $X$ values (or strictly speaking for all $X$ values in the $X \ll 1$ domain of validity of the quasi-1-d asymptotic equations). This corresponds to a pure straight line decay.

If however $1 \ll M_{s} \ll 1 / \Delta_{0}$, then (according to equations (28) and (30)) values of $X$ on the order of $\Delta_{0}^{1 / 2}$ still give very large values of $\partial^{2} U_{s} / \partial X^{2}$, implying significant non-uniformities in $\partial U_{s} / \partial X$. As $X$ grows however, the value of $\partial^{2} U_{s} / \partial X^{2}$ decays very significantly, and it is in this domain that $U_{s}$ vs $X$ is arrested into a straight line decay.

The predictions (when $M_{s} \gg 1 / \Delta$ ) of uniform $\partial U_{s} / \partial X$ in (at least part of) the solution domain only hold for $X \ll 1$ (the domain where the quasi-1-d analysis applies). However for larger $X$ values, analogous arguments imply (via equation (25)) a uniform $\partial U_{s} / \partial S$ (recalling that Cartesian coordinate $X$ and arc length coordinate $S$ coincide for $X \ll 1$ ).

\section{3. $\left|\partial U_{s} / \partial S\right|$ evaluated at the film-Plateau border junction}

The profiles of $U_{s}$ vs $S$ allow us to compute the values of surface strain rate $\left|\partial U_{s} / \partial S\right|$ at the film-Plateau border junction $S=0$. Recall from Section 5.2.3 that knowing these 
values is important for coupling the film and Plateau border flows together.

Tabulated data for $\left|\partial U_{s} / \partial S\right|_{S=0} \mid$ are given in Table 1 (and are also plotted in Figure 9). An alternative way to present the same data (see Table 2) is in terms of the parameter $c$ defined via equation (32) which gives

$$
c=\sqrt{\Delta_{0} M_{s}}\left|\partial U_{s} / \partial S\right|_{S=0} \mid
$$

Figure 9 shows that for sufficiently large values of $M_{s}$, the value of $\left|\partial U_{s} / \partial S\right|_{S=0} \mid$ approaches $6 / \pi$ (just as equation (38) predicts). Then corresponds to a uniform straight line decay of $\left|U_{s}\right|$ from $\left|U_{s}\right|=1$ at $S=0$ (the film-Plateau border entrance) to $\left|U_{s}\right|=0$ at $S=\frac{\pi}{6}$ (the symmetry point on the Plateau border surface). The value of $M_{s}$ at which $\left|\partial U_{s} / \partial S\right|_{S=0} \mid$ becomes close to $6 / \pi$ depends on $\Delta_{0}$, with agreement achieved sooner as $\Delta_{0}$ increases. This finding is in accordance with the predictions of Section 8.2.3 which required large values of $\Delta_{0} M_{s}$ for a pure straight line decay.

The particular data which match these predictions are highlighted in Table 1. Nevertheless the important conclusion from Figure 9 and Table 1 is that for many combinations of $\Delta_{0}$ and $M_{s}$ that we have considered, it is simply not the case that $\left|\partial U_{s} / \partial S\right|_{S=0} \mid$ is close to $6 / \pi$. As a result, the assumption (31) used by Vitasari et al. (2015) to determine the flow velocity at the film-Plateau border junction (and hence the rate of surfactant mass transfer predicted between Plateau border and film) is not always valid. Rescaling the data (as we do in Section 8.3.1 below) to collapse it in various different regimes can help us to deduce how $\left|\partial U_{s} / \partial S\right|_{S=0} \mid$ behaves as a function of the parameters $\Delta_{0}$ and $M_{s}$.

\subsubsection{Rescaling $\left|\partial U_{s} / \partial S\right|_{S=0} \mid$ data}

If we rescale the data from Figure 9 by multiplying $\left|\partial U_{s} / \partial S\right|_{S=0} \mid$ by $\Delta_{0}$ we obtain the data of Figure 10. In the limit of very small $M_{s}$ (i.e. for ${ }^{11} M_{s} \ll \Delta_{0} \ll 1$ ) the rescaled data approach a constant making it apparent that $\left|\partial U_{s} / \partial S\right|_{S=0} \mid$ scales proportionally with

\footnotetext{
${ }^{11}$ In order to achieve $M_{s} \ll \Delta_{0}$, it is necessary to choose the very largest value of $\Delta_{0}$ discussed earlier in Section $5.1\left(\Delta_{0}=4 \times 10^{-2}\right)$, as well as a $M_{s}$ value more than 5 orders of magnitude smaller than the base case value $\left(M_{s}=8800\right.$ in the 'base case' discussed above). Indeed from the discussion of Section 5.1, we expect that $M_{s} \ll \Delta_{0}$ will be a far less common scenario than $\Delta_{0} \ll M_{s}$.
} 
$1 / \Delta_{0}$ (numerically the coefficient of proportionality appears to be close to 7 ).

This corresponds to $U_{s}$ decaying rapidly in space, over an order $\Delta_{0}$ length scale. As explained in Section 5.2.3, that raises a concern as to whether Marangoni stresses can be ignored in the Plateau border as our calculations have done. Even putting that concern to one side for the moment, we note disagreement with the quasi-1-d predictions described in Section 8.1.4 which imply (for $M_{s}$ and $\Delta_{0}$ values both rather smaller than unity) exponential decay on a length scale $\sqrt{\Delta_{0} M_{s} / 3}$. To understand why the exponential decay predictions cease to apply in the limit of very small $M_{s}$ (even in the absence of Marangoni stresses) it is necessary to realise that they were obtained on the basis of lubrication theory, which demands longitudinal variations in the flow only occur on distances greater than the thickness of the flow domain. For an exponential decay to be valid $\sqrt{\Delta_{0} M_{s} / 3}$ should exceed $\Delta_{0}$ (or in order of magnitude terms $M_{s}$ must be at least as large as $\Delta_{0}$ ).

In the limit of $M_{s} \ll \Delta_{0} \ll 1$ the parabolic profile hypothesised in (27) must adjust locally very close to the Plateau border surface (at $Y \approx \Delta$ ) so as to change the magnitude of $|\partial U / \partial Y|$ from the 'parabolic profile' prediction $3 U_{s} / \Delta$ to a much smaller value. Equation (29) then no longer implies (30), and decay of $U_{s}$ is permitted over an order $\Delta_{0}$ distance, instead of the $\sqrt{\Delta_{0} M_{s} / 3}$ distance that equation (30) would predict. In an extreme case i.e. $M_{s} \rightarrow 0$ (see Figure 11), a 'stick-slip' problem would arise (reminiscent of that considered in Richardson (1970)) with a non-zero $\partial U / \partial Y$ on the surface of the film (thanks to a non-zero Marangoni stress there) matching to a vanishing $\partial U / \partial Y$ on the surface of the Plateau border (where Marangoni stresses are, by assumption, neglected).

As mentioned in Section 5.2.3, the Plateau border has barely thickened at all over this tiny spatial distance, and it is not necessarily appropriate to treat the Plateau border in the way our model assumes as being a reservoir of surfactant (with constant and uniform surfactant coverage). In any case when $M_{s}$ is exceedingly small it seems simplest just to ignore surface viscosity altogether, decoupling the Plateau border from the film, and then to compute the surfactant transport processes of interest on the film via the procedures already established in Vitasari et al. (2013b).

Returning to consider the full set of $\Delta_{0}$ and $M_{s}$ values studied in our numerical sim- 
ulations, yet another way of scaling the data is now presented in Figure 12, namely $\Delta_{0}^{1 / 2}\left|\partial U_{s} / \partial S\right|_{S=0} \mid$ plotted against $M_{s}$. The figure makes it apparent that for a range of $M_{s}$ values, more or less those values satisfying $\Delta_{0} \leq M_{s} \leq 1$, we find

$$
\left|\partial U_{s} / \partial S\right|_{S=0} \mid \approx \sqrt{3}\left(\Delta_{0} M_{s}\right)^{-1 / 2}
$$

an equation that follows directly from the exponential velocity decay (with decay length $\sqrt{\Delta_{0} M_{s} / 3}$ ) as predicted in Section 8.1.4. Equation (41) is plotted on Figure 12 (the line marked 'slope $-\frac{1}{2}$ '). In particular for the very smallest $\Delta_{0}$ values that we considered (i.e. $\left.\Delta_{0}=0.00005\right)$, we see good collapse of data onto that line over several decades.

Expressed in terms of the parameter $c$, equation (41) implies that $c=\sqrt{3}$ : data matching this $c$ value are highlighted in Table 2. Knowing $c$ is important for determining the flow at the film-Plateau border junction (which is evident from equation (23), and which is an issue upon which we elaborate later). Computing the 'Plateau border to film' flow raises however a similar concern to the one just noted above: we are still dealing with decays over a length scale sufficiently short (and hence over Plateau border thicknesses sufficiently limited) that it might not be appropriate to use our present model that treats Plateau borders as surfactant reservoirs with constant and uniform surfactant coverage.

Despite the good collapse of data onto the 'slope $-\frac{1}{2}$ ' line noted above, if we take sufficiently large $M_{s}$ values in Figure 12, it is clear that the data begin to lie above that line. This implies that $c$ exceeds $\sqrt{3}$. This is of course expected because for $\Delta_{0} M_{s}$ values in excess of unity we know that $\left|\partial U_{s} / \partial S\right|_{S=0} \mid \approx \frac{6}{\pi}$ and hence (via equation (40)) we have $c \approx \frac{6}{\pi} \sqrt{\Delta_{0} M_{s}}$, which certainly exceeds $\sqrt{3}$.

What is of interest here however is that there is a range of $M_{s}$ values (typically those values with $\left.1 \ll M_{s} \ll 1 / \Delta_{0}\right)$ for which data on Figure 12 lie underneath the line marked 'slope $-\frac{1}{2}$ '. Those data have $c$ values less than $\sqrt{3}$. The smallest $c$ value computed in Table 2 within the parameter regime under consideration occurs for $\Delta_{0}=5 \times 10^{-5}$ and $M_{s}=1000$ and has the value $c=0.616$. Physically $c$ values less than $\sqrt{3}$ imply velocity fields moving into the Plateau border decaying over scales longer than the nominal exponential decay length $\sqrt{\Delta_{0} M_{s} / 3}$. These longer length scales can be associated with 
the frustrated exponential mechanism described in Section 8.1.4. Further discussion of these $c$ values less than $\sqrt{3}$, including a formula for predicting what the $c$ values actually are, can be found in the appendix.

\subsection{Distributions of pressure}

In our numerical analysis we have assumed that the Plateau border surface is a uniform circular arc. This was a simplification that we introduced in Section 3. In reality the Plateau border surface should be able to deform out of circular, with the local curvature and the pressure jump from liquid to gas being linked by a Young-Laplace relation. We can however gauge the suitability (or otherwise) of the circular arc approximation by examining how the pressure in the liquid phase (computed using the circular arc approximation) varies along the Plateau border surface. The more uniform is the computed pressure distribution along that circular arc surface, the better is the circular arc approximation.

Sections 8.4.1 and 8.4.2 consider respectively how the computed pressure distributions are affected by varying $M_{s}$ and $\Delta_{0}$.

\subsubsection{Pressure dependence with respect to $M_{s}$}

Figure 13 plots the absolute value of pressure $|p|$ vs arc length $S$ measured along the Plateau border surface. Recall from Section 5.2.2 that (at least at the Plateau border symmetry point $\left.S=\frac{\pi}{6}\right)$, the value of $p$ is negative, being set to $-\left(\Delta_{0} a^{\prime}\right)^{-1}$ at that symmetry point. Here $\Delta_{0}$ is 0.005 and $a^{\prime}=0.1$, although a number of different values of $M_{s}(10000,10,1$ and 0.1$)$ are considered.

Along the Plateau border surface $r^{\prime}=1$, the arc length $S$ is identical to angular coordinate $\theta$. Even though Figure 13 corresponds specifically to $r^{\prime}=1$, plots of pressure $|p|$ vs coordinate $\theta$ along the Plateau border symmetry line $r^{\prime}=\left(1+\Delta_{0}\right) / \cos \theta$, although not plotted here, actually turn out to look the same (on the scale of the graph). This indicates that $p$ is primarily a function of $\theta$ with comparatively weak dependence on $r^{\prime}$.

The gradient of the pressure, dominated by $\left(r^{\prime}\right)^{-1} \partial p / \partial \theta$, is required to drive Stokes flow within the Plateau border. That a pressure gradient is present is evident in Figure 13: starting from the symmetry point of the Plateau border surface $S=\frac{\pi}{6}$, and then moving 
backwards to smaller $S$ values, the pressure $p$ is seen to rise, i.e. $p$ becomes less negative, and so $|p|$ falls. In certain cases for very small $S$ values, $p$ is predicted to change sign from negative to positive, so that $|p|$ starts to rise again. We will consider the implications of this predicted sign change very shortly, but for the moment we focus solely on pressure gradients. Figure 13 shows the pressure gradient is largest for comparatively small $S$, but decays quite rapidly moving along the Plateau border. Such a decay in the pressure gradient comes about for two reasons: firstly the border thickens (implying via lubrication theory that less pressure gradient is required to drive a given amount of flow along it) and secondly the flow field itself (represented e.g. by the surface velocity $U_{s}$ ) also decays. Hence over much of the Plateau border surface (on the approach to the symmetry point $S=\frac{\pi}{6}$ ) the pressure gradient is negligible, i.e. $|p|$ is near uniform, this uniform value being $\left(\Delta_{0} a^{\prime}\right)^{-1}$ (shown by a dotted line in Figure 13 and representing the pressure jump across the gas-liquid surface of the Plateau border).

Computed $|p|$ values close to this dotted line can be considered to be comparatively close to $\left(\Delta_{0} a^{\prime}\right)^{-1}$, permitting us to treat the corresponding part of the Plateau border surface as a uniformly curved arc. Computed pressures that deviate from the dotted line however, indicate parts of the domain where the Plateau border should not be treated as uniformly curved, i.e. where the shape of the border ought to be adjusted or relaxed. Such pressures occur for decreasing $S$ values moving towards the neighbourhood of the film-Plateau border junction, pointing to the existence of a transition region (as already alluded to in Section 3 and analogous to what is considered in Bretherton (1961); Reinelt and Kraynik (1989, 1990); Schwartz and Princen (1987)) where the shape of the domain needs to adjust between a comparatively flat film and a circular Plateau border. We do not however attempt to compute such transition regions here. An increase in the value of $p$ moving backwards from $S=\frac{\pi}{6}$ (i.e. a decrease in $|p|$ as long as $p$ remains negative) suggests a tendency that would favour a thicker and flatter, lower curvature border had the border been permitted to relax its shape. Predicted sign changes in $p$ from negative to positive are an extreme manifestation of the need to adjust the shape. Although a Young-Laplace law on the gas-liquid surface of the Plateau border is not imposed in our 
computation, were such a law to be imposed, a sign change in the pressure implies a sign change in the surface curvature: assumptions of uniform curvature are then untenable.

Smaller $M_{s}$ values imply smaller and smaller deviation from the dotted line: this reflects the rapid spatial decay (with increasing $S$ ) of the velocity $U_{s}$ at small $M_{s}$, which in turn implies a rapid spatial decay of the pressure gradient $\left(r^{\prime}\right)^{-1} \partial p / \partial \theta$. Indeed for the smallest $M_{s}$ value plotted, the entire pressure distribution lies comparatively close to the dotted line, and no sign change in $p$ is observed.

\subsubsection{Pressure dependence with respect to $\Delta_{0}$}

Section 8.4.1 considered pressures only for the case $\Delta_{0}=0.005$. A decrease in $\Delta_{0}$ increases the magnitude of the pressure. Partly this is due to setting the pressure at the symmetry point $S=\frac{\pi}{6}$ of the Plateau border surface to $-\left(\Delta_{0} a^{\prime}\right)^{-1}$. Even if we compensate for this, by plotting $p+\left(\Delta_{0} a^{\prime}\right)^{-1}$ vs $S$ (instead of $p$ vs $S$ ) we still see higher pressure drops along the Plateau border with smaller $\Delta_{0}$ : see e.g. Figure 14 comparing $\Delta_{0}=0.005$ with $\Delta_{0}=0.00005$. This is unsurprising: a Stokes flow incurs a larger pressure drop in an extremely narrow gap $\left(\Delta_{0}=0.00005\right)$ than in a somewhat wider gap $\left(\Delta_{0}=0.005\right)$.

Values of $M_{s}=10000$ and $M_{s}=1$ are shown in Figure 14. For $M_{s}=10000$ the pressure distributions (when expressed in terms of $p+\left(\Delta_{0} a^{\prime}\right)^{-1}$ instead of $p$ ) converge together for $S$ values greater than about 0.2 regardless of the value of $\Delta_{0}$. This is expected, because the surface velocity distribution in these $M_{s}=10000$ cases should follow the straight line function equation (38) regardless of the value of $\Delta_{0}$. Away from the narrow gap region at the entrance to the Plateau border, the same surface velocity distribution on effectively the same solution domain must produce the same gradients of pressure.

For $M_{s}=1$ we do not see the $\Delta_{0}=0.005$ and $\Delta_{0}=0.00005$ pressure distributions converging together in the fashion that was observed for $M_{s}=10000$. The pressure drop to drive a Stokes flow depends not just on the geometry of the flow domain, but also on the velocity field within the domain. In Figure 8 we see that the velocity distribution for $\Delta_{0}=0.00005$ and $M_{s}=1$ decays to zero far more quickly as $S$ grows than the distribution for $\Delta_{0}=0.005$ and $M_{s}=1$ does. The velocity field for $\Delta_{0}=0.005$ and $M_{s}=1$ shows an initial rapid decay being replaced further along the border by a much more gradual 
one. Since the velocity field for $\Delta_{0}=0.005$ and $M_{s}=1$ survives longer than that for $\Delta_{0}=0.00005$ and $M_{s}=1$, larger pressures are expected with the larger $\Delta_{0}$ value.

We have marked on Figure 14 lines corresponding to zero pressure, so that $p+\left(\Delta a^{\prime}\right)^{-1}$ becomes the same as $\left(\Delta_{0} a^{\prime}\right)^{-1}$ : see the dashed line (for the case $\Delta_{0}=0.005$ ) or the dotted line (for the case $\Delta_{0}=0.00005$ ). As was the case in Figure 13, over much of the domain, pressures are below these lines (indicating a modest change in the value of $|p|$ relative to $\left(\Delta_{0} a^{\prime}\right)^{-1}$, and hence a Plateau border surface that is well approximated by a uniformly curved arc). It is only for very small values of $S$ that the computed pressures for any given $\Delta_{0}$ lie above the respective dashed or dotted line, indicating a need to relax the shape of the Plateau border surface away from a circular arc.

\subsection{Surfactant mass transfer rate from Plateau border to film}

The velocity data of Section 8.2 have been scaled such that the dimensionless speed at the film-Plateau border junction $\left|U_{s}\right|_{S=0} \mid$ was equal to unity for all values of $\Delta_{0}$ and $M_{s}$. This scaling is very convenient for solving for the Plateau border velocity field in isolation from the film. Here however we want to estimate the magnitude of $\left|u_{s}^{\prime}\right|_{S=0} \mid$, i.e. the speed at the film-Plateau border non-dimensionalised on the velocity scale specified in Section 5.2.1. This alternate scaling (in terms of $u_{s}^{\prime}$ rather than $U_{s}$ ) allows us to couple the Plateau border and film together, specifically to determine the border to film surfactant transport rate as a function of $\Delta_{0}$ and $M_{s}$, to reveal whether there are particular parameter regimes where the Plateau border is acting as a bottleneck for the flow onto the film.

Equation (23) actually gives us not the value of $\left|u_{s}^{\prime}\right|_{S=0} \mid$ on its own, but rather the value of the ratio $\left|u_{s}^{\prime}\right|_{S=0}|/| u_{s(o)}^{\prime} \mid$ (with both $\left.u_{s}^{\prime}\right|_{S=0}$ and $u_{s(o)}^{\prime}$ expected to be negative quantities as surfactant flow is away from the Plateau border towards the centre of the film). We do however expect that $\left|u_{s(o)}^{\prime}\right|$ (the speed of the film outside a velocity boundary layer, and hence unconstrained by the border) will be order unity. This follows as a result of the way $u_{s}^{\prime}$ has been non-dimensionalised as long as $\Delta_{0} M_{s} \leq\left(a^{\prime}\right)^{-2}$ and as long as variations in surfactant coverage along the film are of comparable magnitude to the surfactant coverage on the Plateau border itself (Vitasari et al., 2015).

Since $a^{\prime}$ is a small parameter (we assume $a^{\prime}=0.1$ here), the above mentioned constraint 
$\Delta_{0} M_{s} \leq\left(a^{\prime}\right)^{-2}$ is satisfied for all $\Delta_{0}$ and $M_{s}$ values considered in Table 1 , with the exception of the product of the largest $\Delta_{0}$ and $M_{s}$ values $\left(0.05\right.$ and $10^{4}$ respectively $\left.{ }^{12}\right)$. Given an order unity value of $\left|u_{s(o)}^{\prime}\right|$ as mentioned above, it follows from equation (23) that the 'Plateau border to film' flow $\left|u_{s}^{\prime}\right|_{S=0} \mid$ will be order $\sqrt{3} /(\sqrt{3}+c)$. In other words, the larger the value of $c$, the greater the tendency of the Plateau border to act as a bottleneck.

In Sections 8.5.1, 8.5.2 and 8.5.3 that follow we consider how $\left|u_{s}^{\prime}\right|_{S=0} \mid$ behaves for various combinations of the parameters $M_{s}$ and $\Delta_{0}$. Sections 8.5.4-8.5.6 then summarise and discuss these findings and relate them to time scales for mass transfer.

\subsubsection{Value of $\left|u_{s}^{\prime}\right|_{S=0} \mid$ in case $M_{s} \gg 1 / \Delta_{0}$}

In the event that $\Delta_{0} M_{s} \gg O(1)$ (still of course with $\Delta_{0} M_{s}$ rather smaller than $\left(a^{\prime}\right)^{-2}$ ) it happens that $c=\frac{6}{\pi} \sqrt{\Delta_{0} M_{s}}$, as we have already established in Section 8.3.1. Clearly $c \gg 1$ in this particular limit. Substituting into equation (23), then suggests $\left|u_{s}^{\prime}\right|_{S=0}|\approx(\sqrt{3} / c)| u_{s(o)}^{\prime} \mid$ (still taking $\left|u_{s(o)}^{\prime}\right|$ as being order unity). Based on the definition in equation (14), this rearranges to $\left|u_{s}^{\prime}\right|_{S=0}\left|\approx\left(a^{\prime} / a_{c r i t}^{\prime}\right)\right| u_{s(o)}^{\prime} \mid$ with $a^{\prime} \ll a_{c r i t}^{\prime}$ whenever $\Delta_{0} M_{s} \gg O(1)$. In a typical case e.g. $M_{s}=10^{4}$ and $\Delta_{0}=0.005$ (such as was considered in the streamline pattern shown in Figure 5(a)) we deduce via equation (14) that $a_{\text {crit }}^{\prime} \approx 0.78$. Hence $a^{\prime} / a_{c r i t}^{\prime}$ which is equal to $(\sqrt{3} \pi / 6)\left(\Delta_{0} M_{s}\right)^{-1 / 2}$ evaluates to roughly 0.12 for these parameter values. The speed at the film-Plateau border junction $\left|u_{s}^{\prime}\right|_{S=0} \mid$ is therefore an order of magnitude smaller than the typical film velocity $\left|u_{s(o)}^{\prime}\right|$. The above situation implies that either the foam is sufficiently dry (i.e. the Plateau border size relative to the film measured via the parameter $a^{\prime}$ is sufficiently small) and/or the surface viscosity is sufficiently large (or in other words $a_{c r i t}^{\prime}$ is sufficiently large), that the zero velocity constraint on the symmetry point of the Plateau border manages to extend its influence all the way to the film-Plateau border junction.

\footnotetext{
${ }^{12}$ It has been shown (Vitasari et al., 2015) that any $\Delta_{0}$ and $M_{s}$ combination such that the product $\Delta_{0} M_{s}$ exceeds unity automatically reduced $\left|u_{s}^{\prime}\right|_{S=0} \mid$ down to an order $1 / \sqrt{\Delta_{0} M_{s}}$ value. In the event that in addition $\Delta_{0} M_{s} \geq\left(a^{\prime}\right)^{-2}$ (so that $1 / \sqrt{\Delta_{0} M_{s}}$ is even less than the already small parameter $a^{\prime}$ ), a theory of Vitasari et al. (2015) suggests further reductions in $\left|u_{s}^{\prime}\right|_{S=0} \mid$ over and above the aforementioned ones.
} 


\subsubsection{Value of $\left|u_{s}^{\prime}\right|_{S=0} \mid$ in case $\Delta_{0} \ll M_{s} \ll 1$}

Now consider the case $\Delta_{0} \ll M_{s} \ll 1$. In this case we predict (see e.g. Figure 12) that $c=\sqrt{3}$. According to equation (23) the flow speed at the film-Plateau border junction is half of the nominal film surface speed $\left|u_{s(o)}^{\prime}\right|$ (itself an order unity quantity) that applies to film points away from the Plateau border. The surface viscosity is now sufficiently low that the junction is 'unaware' of the exact location of the Plateau border symmetry point, and so is not constrained by that point. However the halving of the film velocity arises from the assumed lack of any Marangoni stress contribution from the Plateau border. As already mentioned previously that assumption may well be invalid when $\Delta_{0} \ll M_{s} \ll 1$, and restoring Marangoni stresses on that part of the Plateau border which is only marginally thicker than the film will restore $\left.u_{s}^{\prime}\right|_{S=0}$ back to the level of $u_{s(o)}^{\prime}$.

\subsubsection{Value of $\left|u_{s}^{\prime}\right|_{S=0} \mid$ in case $1 \ll M_{s} \ll 1 / \Delta_{0}$}

Finally consider a value $c \leq \sqrt{3}$ as occurs in the domain $1 \ll M_{s} \ll 1 / \Delta_{0}$. For example consider the value $c=0.616$ corresponding (as mentioned in Section 8.3.1 above) to $\Delta_{0}=5 \times 10^{-5}$ and $M_{s}=1000$. Via equation (23), the speed $\left|u_{s}^{\prime}\right|_{S=0} \mid$ at the film-Plateau border junction is now 0.73 times the nominal film speed $\left|u_{s(o)}^{\prime}\right|$ away from the border. The fact that the velocity at the junction is less than that in the film once again arises due to assuming no Marangoni stresses in the Plateau border: this tends to reduce the velocity at the film-Plateau border junction relative to $u_{s(o)}^{\prime}$. However the adverse effect on the speed at the junction is less than previously (i.e. multiplication by a factor 0.73 instead of by a factor of a half). The surface viscosity is now at a level where it couples the motion of the film-Plateau border junction with the motion of points on the Plateau border surface where the border is already much thicker than the film, that thicker border restricting the flow less than a thinner border (still assuming no Marangoni stresses) would.

\subsubsection{Time scales for surfactant mass transfer}

To summarise, depending on the $\Delta_{0}$ and $M_{s}$ values considered, and assuming we ignore Marangoni stresses on the Plateau border compared to those on the film (an assumption that could be queried in the case of Section 8.5.2 in particular), we have deduced 
flow velocities slowing down at the film-Plateau border junction by factors of 0.12 (Section 8.5.1), a half (Section 8.5.2) and 0.73 (Section 8.5.3) depending on the values of $\Delta_{0}$ and $M_{s}$ that are chosen. Time required for surfactant transport should scale inversely with those velocities, and would therefore increase by factors of roughly $8.3,2$ and 1.3 respectively. Surface viscosity could thereby make the fractionation process less efficient, in particular if the time required to achieve surfactant transport onto the foam film becomes comparable with the typical residence time of films within the fractionation column.

In dimensional units, bubble residence time in a typical fractionation column has been given (Vitasari et al., 2013b) as around 12 s (based on an experimental study of Martin et al. (2010)). Meanwhile the Marangoni-driven surfactant transfer time scale (converted back to dimensional units, and ignoring any surface viscous effects in the first instance (Vitasari et al., 2013b)) has been estimated to be on the order of $3 \times 10^{-2} \mathrm{~s}$ assuming a comparatively thick film (specifically assuming $\Delta_{0} \approx 4 \times 10^{-2}$ as quoted in Section 5.1). Recalling however that the characteristic velocity given in Section 5.2.1 scales proportional to $\delta_{0}^{\prime} \equiv \Delta_{0} a^{\prime}$, and that characteristic time scales inversely with velocity, reduction in $\Delta_{0}$ by roughly an order of magnitude (to attain the value $\Delta_{0}=0.005$ matching that considered in Section 8.5.1) implies an order of magnitude increase in mass transfer time scale (which becomes roughly $0.3 \mathrm{~s}$ still ignoring surface viscosity). Even if this time scale is increased by the factor 8.3 mentioned above (to account for surface viscous effects) mass transfer should have ample opportunity to occur for any reasonable bubble residence time in a fractionation column (around $12 \mathrm{~s}$ as quoted above). Much smaller $\Delta_{0}$ values (i.e. much thinner films) however change the picture dramatically. A value of $\Delta_{0}$ as low as $5 \times 10^{-5}$ (as in Section 8.5.4) implies a three order of magnitude increase in the estimate of typical surfactant mass transfer time compared to the original time scale $3 \times 10^{-2}$ s quoted above. The surfactant mass transfer time scale then becomes around $30 \mathrm{~s}$ neglecting surface viscous effects. This is now rather longer than the typical residence time in a fractionation column and suggests a redesign requirement using a taller column in order to increase residence time. Surface viscous effects causing further increases in the mass transfer time scale (even quite moderate increases, e.g. doubling 
the time scale or multiplying it by a factor 1.3 as alluded to above) could however impact on the efficiency of even that redesigned column.

Ironically then, cases for which surface viscosity leads to the biggest relative increase in surfactant mass transfer have little impact on the fractionation process overall (because mass transfer time in the absence of surface viscous effects was actually exceedingly short, given the films were still comparatively thick). Cases where surface viscosity produces rather modest increases in already comparatively long mass transfer time scales (which arise in turn owing to having exceedingly thin films) potentially are more problematic. Determining which of these two different film thickness regimes is most applicable to a given fractionation process requires knowledge of film drainage rates. This is beyond the scope of the present discussion (film drainage not being considered here) but the issue is discussed by Vitasari et al. (2013b).

\subsubsection{Coupling to the surfactant concentration field}

Yet another point to note is that a significant velocity difference (as our models suggest via equation (23)) between the film-Plateau border junction and points on the film slightly away from that junction (i.e. immediately outside a 'velocity boundary layer' as has been described in Section 6) has implications for the time evolution of the surfactant concentration field. Detailed analysis of this time evolution is outside the scope of the present work (which is concerned solely with finding instantaneous surfactant transport rates for a given instantaneous surfactant distribution). However the question is relevant for determining mass transfer time scales and so is discussed in qualitative terms below.

Consider an element of film surface immediately adjacent to the film-Plateau border junction, the element size being comparable with the extent of the aforementioned velocity boundary layer. Analogously with a mechanism noted by Vitasari et al. (2015), sharp gradients of surfactant coverage could develop in that film element over time due to the velocity mismatch across it. With that velocity mismatch present but without those sharp surfactant coverage gradients, more surfactant will be leaving the element (driven by Marangoni stresses in the direction towards the film centre) than entering it (from the Plateau border), sharpening gradients of surfactant coverage within the element. Only by 
acquiring a surfactant coverage mismatch almost counterbalancing the velocity mismatch can a near uniform surfactant flux be delivered across the film element in question, which is what is expected for an element that is small compared to the overall length of the film.

Our analysis of film velocity fields ought to be reformulated in cases where sharp surfactant gradients arise on films. At present the analysis starting from equation (13) and leading eventually to equation (23) has assumed a simple structure for the velocity boundary layer on the film, balancing bulk viscous shear stresses with surface viscous stresses within that layer. If however the surfactant concentration field in the film develops a boundary layer character near the junction with the Plateau border (and hence the Marangoni stress field $\partial \gamma^{\prime} / \partial x^{\prime}$ likewise has a boundary layer character), then the velocity boundary layer in the film necessarily becomes much more complex than before. That equation (13) fails to capture any Marangoni effects within the velocity boundary layer is apparent from the discussion of Section 4.2 which indicates that the only material properties affecting the (dimensional) length scale of the velocity boundary layer are $\mu$ and $\mu_{s}$ (i.e. bulk and surface viscosity), the remaining terms contributing to the said length scale being wholly geometric. An equation such as (13) that evaluates the strain rate in the velocity boundary layer at the junction point with the Plateau border wholly in terms of the change in velocity across the boundary layer and the layer's nominal thickness determined without reference to the Marangoni stresses within it, cannot take account of any complex boundary layer structure on the part of the surfactant concentration field.

Since equation (13) can be modified by sharp gradients in the surfactant coverage field, it follows that equation (23) will likewise be modified, as the former equation was needed to derive the latter (see e.g. the derivation of equation (18) of which (23) is merely a dimensionless version). A change in that latter equation has however two important consequences. Firstly it changes the velocity at the film-Plateau border junction (hence changing the border to film surfactant transfer rate, and thereby the time scale to achieve that transfer). Secondly it identifies an important feedback mechanism: modifying equation (23) modifies the velocity mismatch, yet it is that very mismatch which causes (analogously to Vitasari et al. (2015)) a complex structure to appear in the Marangoni 
stress field that in turn required a modification to equation (23) in the first place.

In summary, the predictions of the ratio between surface strain rate and surface velocity within the Plateau border which we have calculated (as functions of $\Delta_{0}$ and $M_{s}$ given in e.g. Table 1) are expected to remain valid. Likewise it is still the case that the surface strain rate and surface velocity are continuous across the film-Plateau border junction. However the velocity mismatch between that junction and points on the film immediately outside a velocity boundary layer produces evolutions of surfactant concentrations that in turn lead to quite complex 'boundary layer' structures for the surfactant distributions along the film with sharp gradients in surfactant coverage being sustained. These complex surfactant distributions can then affect the velocities at the junction, the Plateau border to film mass transfer rates, and the time scale required for mass transfer. Moreover they feed back onto the velocity mismatch that originally produced them.

\subsubsection{Coupling to the shape of the Plateau border}

Section 8.5.5 considered the possibility that the surfactant concentration field might develop a complex structure in the neighbourhood of the film-Plateau border junction, which then affects the local surface velocity and local mass transfer rate at that point. Yet another complication affecting velocity and mass transfer near this junction is that, under the action of normal stresses, the shape of the Plateau border might need to be relaxed away from circularity: see the discussion in Section 8.4.

The key to determining the Plateau border to film mass transfer rates here, has been the computations of surface strain rates at the film-Plateau border junction $\left|\partial U_{s} / \partial S\right|_{S=0} \mid$ (see e.g. Section 8.3), coupling the film and Plateau border flows together.

The surface strain rates were however computed based on assuming a uniformly curved Plateau border surface. It is unclear to what extent relaxing the surface shape in the normal direction is likely to affect these (tangential) surface strain rates. We can hypothesise that if the surface were to be permitted to deflect outwards (in order to relax the higher liquid pressures computed in the neighbourhood of $S=0$ compared to the negative pressures obtained at $S=\frac{\pi}{6}$ ) we would obtain results qualitatively similar to those we already have, merely replacing the film thickness parameter $\Delta_{0}$ by a larger effective value 
to represent the outwards deflection. This is however speculative, and investigating this hypothesis by a full 'free surface' numerical simulation (i.e. allowing the solution domain itself to deform so as to balance normal stresses) represents a considerable undertaking.

\section{Discussion}

The results for the Plateau border flow fields as computed in Section 8 indicate how to match film and Plateau border flows in various different parameter regimes of dimensionless film thickness $\Delta_{0}$ and dimensionless surface viscosity $M_{s}$. The results also indicate the extent to which the presence of the Plateau border constrains the film flow.

Specifically if $\Delta_{0} M_{s} \gg 1$ (requiring an extremely large $M_{s}$ given that $\Delta_{0} \ll 1$ ) the flow at the film-Plateau border junction is very strongly constrained by surface viscous effects (see e.g. equation (21)). Moreover the surface strain rate on the Plateau border is spatially uniform, ensuring that the surface velocity exhibits a uniform straight line decay along the border. This corroborates the work of Vitasari et al. (2015).

In the opposite case of a small $M_{s}$ value, typically for $\Delta_{0} \ll M_{s} \ll 1$, the decay of the velocity field along the Plateau border surface is predicted to be exponential, with a characteristic decay distance predicted by equation (39) to be $\sqrt{\Delta_{0} M_{s} / 3}$, which is now substantially less than the full arc length measured along the border. Via equation (23), this leads to a more constrained velocity at the film-Plateau border junction than the assumption of Vitasari et al. (2015), i.e. equation (31) (in place of equation (32)) would have done. The reason for this is that equation (31) solely constrains the flow at the filmPlateau border junction for geometric reasons, i.e. based on how close by the junction is to the border's symmetry point. In fact for $\Delta_{0} \ll M_{s} \ll 1$ the velocity on the Plateau border decays very near the border entrance, well before approaching that symmetry point, meaning the geometric constraint is not then relevant. The constraint that equation (32) places on the system in the $\Delta_{0} \ll M_{s} \ll 1$ limit arises in fact from the assumed absence of any Marangoni stress on the Plateau border. This then predicts a velocity decay over a much smaller longitudinal distance than a purely geometric symmetry constraint would.

We can nonetheless identify a potential problem with the assumptions underlying our 
model in this case: we have assumed that Marangoni stresses are present on the film, but absent on the Plateau border, on the supposition that the Plateau border contains a reservoir of surfactant through being for the most part substantially thicker than the film. However with that assumption the predicted flow field in the Plateau border is effectively confined to a very small part of the border, specifically the region near the entrance to the border where it is not substantially thicker than the film. Marangoni stresses could still be significant in that particular region, even though in the rest of the border there are insignificant Marangoni stresses and likewise insignificant flow.

Once Marangoni stresses on the Plateau border need to be taken into account, we can no longer solve for the detailed flow field in the Plateau border without prior knowledge of the instantaneous surfactant concentration distribution (and hence instantaneous Marangoni stress field) along the border's surface: we have a coupled fluid mechanical and mass transfer problem for the border in addition to that already studied (Vitasari et al., 2015) for the film. In the presence of Plateau border Marangoni stresses, no constraining effect of the Plateau border would be evident at the junction with the film, constraints only manifesting themselves moving along the border once it becomes much thicker than the film ${ }^{13}$, giving in effect a surfactant reservoir that suppresses Marangoni stresses. Given the order $\sqrt{\Delta_{0}}$ length scale over which the border is predicted to thicken and hence Marangoni stresses would be permitted to decay is now rather greater than the order $\sqrt{\Delta_{0} M_{s}}$ scale over which surface viscosity couples the motion of adjacent surface points together, the flow velocity on the Plateau border surface can be obtained entirely in terms of local properties (local Marangoni stress at a point and local border thickness at that point) ignoring surface viscous effects. This means that the the Plateau border flow no longer couples to the film flow, and hence no longer constrains the film flow. Under those circumstances, in the limit of small $M_{s}$, we can deduce a velocity field on the border $u_{s}^{\prime} \approx \frac{1}{3}\left(a^{\prime}\right)^{-1}\left(\Delta / \Delta_{0}\right) \partial \gamma^{\prime} / \partial X$ (which turns out to be the same velocity field as if we ignored surface viscosity altogether (Vitasari et al., 2013b), the velocity decaying

\footnotetext{
${ }^{13}$ Recall that, as has been explained in Section 3.5, the border will act as a surfactant reservoir once its local thickness exceeds an 'effective Henry constant'. This 'effective Henry constant' is assumed to be intermediate between the film thickness and the curvature radius of the Plateau border surface.
} 
with $X$ moving along the border, provided that $\partial \gamma^{\prime} / \partial X$ decays more rapidly than $\Delta / \Delta_{0}$ grows). Film flows can then be computed in a similar (i.e. entirely local) fashion ignoring surface viscous effects altogether. As far as film flows are concerned, we therefore recover the model already studied in Vitasari et al. (2013b). The above constitutes the most important finding for the case of small $M_{s}$.

To summarise then for $\Delta_{0} \ll M_{s} \ll 1$, equation (23) that implies a velocity at the film-Plateau border junction half of the value 'unconstrained by the border' would have been correct in the present limit $1 \ll \Delta_{0} \ll M_{s}$ if we could contrive to have Marangoni stresses on the film but not on the Plateau border. It is however not necessarily realistic to suppose that there are no Marangoni stresses on the Plateau border in this particular limit. These Plateau border Marangoni stresses return the velocity at the film-Plateau border junction back towards the 'unconstrained' value.

Yet another important limit that we investigated within Section 8 had $\Delta_{0} \ll 1$ and $M_{s} \gg 1$ (but still with $\Delta_{0} M_{s} \ll 1$ and hence $M_{s} \ll 1 / \Delta_{0}$ ). Here the decay in surface velocity along the border is slower than the increase of the border thickness that drives a decay in Marangoni stresses, implying it is actually reasonable to ignore Marangoni effects in the Plateau border, but nonetheless the reduction in the flow at the film-Plateau border junction is less marked than before: the presence of the Plateau border causes the junction velocity to fall but it remains more than half the value that would apply ignoring the constraining effect of the Plateau border. The flow field along the Plateau border no longer decays exponentially. Instead it exhibits a non-uniform decay near the entrance to the Plateau border, and a uniform straight line decay further on.

\section{Conclusions}

In this work we have considered the Marangoni-driven flow and surfactant transport between a foam Plateau border and a foam film during the process of foam fractionation, such flows being likely to arise in various scenarios (e.g. foam fractionation with reflux, fractionation in stripping mode, multicomponent fractionation). In particular we have studied the role that surface viscosity (measured by a dimensionless parameter $M_{s}$ ) and 
film thickness (measured by a dimensionless parameter $\Delta_{0}$ ) play in this flow and mass transfer process. Our analysis recognises that the surface strain rate and the surface velocity must both be continuous across the film-Plateau border junction. Indeed, if we know the ratio between the surface strain rate and the surface velocity at the film-Plateau border junction this provides us with sufficient information to compute the film flow field.

The aforementioned strain rate to velocity ratio can however be obtained via a fluid mechanical analysis in the Plateau border: this ratio is then what couples the Plateau border flow to the film flow. It has been proposed in literature (Vitasari et al., 2015) that the surface strain rate should be uniform on the entire Plateau border surface, and hence that surface velocity needs to be a straight line function of distance along the border, vanishing at a stationary symmetry point on the border surface. The ratio between the surface strain rate and surface velocity (at the film-Plateau border junction) is then just the reciprocal of arc length of surface between the symmetry point and the junction.

Our fluid mechanical analysis shows that this hypothesis is indeed valid in the case when the product $\Delta_{0} M_{s}$ exceeds unity. Surface viscosity is then sufficiently strong that the film-Plateau border junction is constrained by the stationary symmetry point mentioned above: the velocity at the film-Plateau border junction is reduced substantially compared to the 'no surface viscosity' case and the surfactant mass transfer process slows down significantly. The impact on the total mass transfer taking place in a fractionation column can be surprisingly little however, since these substantial slow downs in transfer rate typically occur in situations where foam films are sufficiently thick that the characteristic mass transfer time scale (prior to the 'surface viscous' slow down) is orders of magnitude less than the available residence time that foam films spend in the fractionation column.

In other parameter regimes (i.e. $\Delta_{0} M_{s} \ll 1$ ) significant non-uniformities are possible in the surface strain rate along the Plateau border length (a contrast from what was hypothesised by Vitasari et al. (2015)). The case $\Delta_{0} M_{s} \ll 1$ could be further subdivided into $\Delta_{0} \ll M_{s} \ll 1$ and $1 \ll M_{s} \ll 1 / \Delta_{0}$. In the case $\Delta_{0} \ll M_{s} \ll 1$ a rapid exponential spatial decay of surface strain rate and surface velocity is predicted along the Plateau border, and such decay is clearly spatially non-uniform. In the case $1 \ll M_{s} \ll 1 / \Delta_{0}$ 
on the other hand, a rapid and non-uniform spatial decay occurs near the film-Plateau border junction, but this is then arrested and replaced by a uniform decay further along the border. There are consequences for the velocity of surfactant mass transfer across the film-Plateau border junction, being reduced to half of its 'no surface viscosity' value in one case, and somewhat more than half of that value in the other. Time scales for surfactant transfer are thereby increased by a factor of two in the first case, and by a factor somewhat less than two in the other. These moderate increases in surfactant mass transfer times may however impact negatively on the efficiency of the foam fractionation process as they typically occur in regimes with exceedingly thin foam films, when transfer (even without the complications of surface viscosity) is slow, and struggles to reach completion within the residence time available to foam films within the fractionation column.

In the regime $\Delta_{0} M_{s} \ll 1$, the lower velocities due to surface viscosity referred to above arise not from constraints imposed at the Plateau border symmetry point, but rather from the fact that the Plateau border is assumed to act as a constant and uniform surfactant reservoir, thereby suppressing Marangoni stresses on the entire border. One could query this assumption in e.g. the case $M_{s} \ll 1$ in particular, because in that case significant surface velocities turn out to be confined to a very restricted part of the Plateau border near the film-Plateau border junction, where the border thickness is comparable with that of the film, and insufficient to contain a surfactant reservoir. In such cases, the flow on the film is unlikely to be constrained by the presence of the Plateau border.

The analysis that we have presented here can compute velocity fields and thereby surfactant flux fields on films and Plateau borders at a given instant in time and for a given instantaneous surfactant coverage distribution (surfactant being considered to vary much more significantly on the films than on the Plateau borders which are treated as surfactant reservoirs as mentioned above). We have not in this work attempted to compute the time evolution of the surfactant coverage on the film produced by these velocity and flux fields. We cannot rule out the possibility therefore that the surfactant coverage field will evolve over time in such a way as to invalidate some of the assumptions we have used to obtain the above mentioned estimates of velocities at film-Plateau border junctions, 
of associated surfactant mass transfer rates, and hence of surfactant mass transfer time scales. The predicted ratios between surface strain rates and surface velocities at the filmPlateau border junction (which suffice to close the governing equations for the film) are likely to be more robust than the estimates we have given of surface velocity itself. This is because deriving those estimates of surface velocity involves additional constraining assumptions about how surfactant might be distributed on films.

Throughout our work a simplifying assumption has been that the domain of the filmPlateau border system is fixed with a planar film joining up directly with a circular arc border. The pressure jumps across the gas-liquid Plateau border surface can be estimated in such a system and compared with the pressure distribution in the liquid phase along the assumed circular arc border. Over much of the assumed circular arc, the computed pressure distribution in the liquid is sufficiently uniform compared to the size of the aforementioned pressure jumps that deviations from circularity can be neglected. This is not however the case near the film-Plateau border junction where a transition region is required over which the surface is not a circular arc: indeed the location of the surface is not known a priori. Computing the transition region is beyond the scope of this study, involving modifications to the flow domain applying a boundary condition in the normal direction. This is however precisely the region where we need to extract tangential surface information, i.e. the ratio between surface strain rate and surface velocity, which we use to couple the Plateau border to the film. The effect of including a transition region upon these tangential properties is unclear, but given that the transition region is expected to thicken the Plateau border near the point where it joins up with the film, the effect may be simply like increasing the value of $\Delta_{0}$ in the present model.

\section{Acknowledgements}

PG acknowledges sabbatical stay funding from CONICET Argentina res. no. 2218/13 and from CONICYT Chile folio 80140040. 


\section{Appendix A. Boundary conditions at the Plateau border entrance}

This appendix explains some of the subtleties (alluded to in Section 3.3) associated with identifying a suitable boundary condition across the Plateau border entrance.

The thin geometry of the film (with film aspect ratio $\delta_{0}^{\prime}$ defined as $\delta_{0}^{\prime} \equiv \delta_{0} / L \ll 1$ ) suggests a requirement that, in the polar coordinates of the Plateau border, $\left|u_{\theta}\right| \gg\left|u_{r}\right|$. This is not the same as $u_{r}$ being identically zero everywhere along $\theta=0$. Indeed imposing a condition that $u_{r}$ vanishes along $\theta=0$ (and hence $\partial u_{r} / \partial r$ also vanishes there) leads to problems at the point $r=a, \theta=0$. Approaching that particular point along the Plateau border surface (i.e. along $r=a$ ), the surface strain rate $\partial u_{s} / \partial s=a^{-1} \partial u_{s} / \partial \theta$ must be non-zero: indeed determining the value of $\partial u_{s} / \partial s$ is important for coupling the film and Plateau border flows. If however (approached along $\theta=0$ ), the value of $u_{r}$ and likewise the value of $\partial u_{r} / \partial r$ are taken to vanish at that same point, then continuity is violated.

In order to understand why imposing a condition $u_{r}=0$ is problematic at the filmPlateau border junction, it is useful to consider more carefully the velocity profile in the film, viz. a parabolic flow profile such as equation (1). If $u_{s}$ happens to be changing along the film surface, it is actually possible to compute the non-zero velocity component in the transverse direction merely via the continuity equation. The fact that we are able to use a continuity equation to deduce the transverse velocity component, rather than more conventionally invoking a transverse momentum equation, comes about because of the extreme aspect ratio of the film (i.e. $\delta_{0}^{\prime} \ll 1$ implying $\Delta_{0} \ll 1$ also). For such an aspect ratio, the transverse component of the momentum equation is trivial in the film (merely stating that pressure gradients are longitudinal rather than transverse). As one moves from the film deeper and deeper into the Plateau border however, eventually the transverse component of the momentum equation must cease to be trivial: lubrication type assumptions must eventually cease to apply. Hence to determine the Plateau border flow field, a second boundary condition is required along $\theta=0$ to accompany the nontrivial transverse momentum equation.

Near the entrance to the Plateau border, based on geometry, we have already stated that $\left|u_{\theta}\right| \gg\left|u_{r}\right|$. Moreover (again due to geometry) the radial derivative of $u_{\theta}$ (i.e. 
$\left.\partial u_{\theta} / \partial r\right)$ is expected to be vastly in excess of the angular derivative of $u_{r}$ (i.e. $r^{-1} \partial u_{r} / \partial \theta$ ). Given equation (8) for $u_{\theta}$ vs $r$, the value of $\partial u_{\theta} / \partial r$ is known, whereas $r^{-1} \partial u_{r} / \partial \theta$ is a priori unknown. Now the $r, \theta$ component of the strain rate tensor (i.e. the stress tensor divided by viscosity $\mu$ ) is $\partial u_{\theta} / \partial r-u_{\theta} / r+r^{-1} \partial u_{r} / \partial \theta$. Based on the above arguments we already anticipate $\partial u_{\theta} / \partial r$ dominates $r^{-1} \partial u_{r} / \partial \theta$. The geometry also implies that $-u_{\theta} / r$ is smaller than $\partial u_{\theta} / \partial r$ (by a factor on the order of $\Delta_{0}$ ).

We therefore decided to implement a stress boundary condition setting the $r, \theta$ stress component equal to the known term $\mu \partial u_{\theta} / \partial r$ which is expected to dominate. This boundary condition permits computation of flow fields avoiding any problems satisfying continuity at the point $r=a, \theta=0$.

One special situation that we can use to illustrate our chosen stress boundary condition is the hypothetical case for which flow in the Plateau border is purely rectilinear in the direction parallel to the film and also locally invariant along that direction. The terms we have neglected from the strain rate $-u_{\theta} / r$ and $r^{-1} \partial u_{r} / \partial \theta$ then turn out to cancel one another exactly. This is a manifestation of the fact that the radial velocity component can change with $\theta$ simply because the radial unit vector changes with $\theta$, even if the direction of the fluid velocity vector itself remains fixed. However in this special case, the aforementioned invariance of the assumed rectilinear flow also implies (via continuity) that $u_{r}$ vanishes at $\theta=0$. The above is of course a very special case: under ordinary circumstances we expect local tangential variation of the flow along the Plateau border and our chosen boundary condition then produces a non-zero $u_{r}$.

\section{Appendix B. Case of large $M_{s}$ with $1 \ll M_{s} \ll 1 / \Delta_{0}$}

In the main text we indicated how for large values of the parameter $\Delta_{0} M_{s} \gg 1$, the flow field $U_{s}$ on the Plateau border surface was well represented by a straight line function (38) that decayed uniformly between the entrance to the Plateau border and the border's symmetry point. Meanwhile for small values of $\Delta_{0} M_{s}$ with $\Delta_{0} \ll M_{s} \ll 1$, we indicated (in Section 8.1.4) how an asymptotic analysis near the Plateau border entrance predicted an exponential decay of $U_{s}$ on a characteristic length scale much smaller than unity. 
Cases with $1 \ll M_{s} \ll 1 / \Delta_{0}$ correspond to neither of the above mentioned limits. Such cases are nonetheless of physical interest. Section 5.1 suggests that $M_{s}$ could be as large as 8800 for fractionation of a high surface viscosity surface active protein, such as bovine serum albumin (BSA), whereas $\Delta_{0}$ could be as low as $3 \times 10^{-5}$ for a common black film (giving $1 / \Delta_{0}$ on the order of 33000 ).

For $1 \ll M_{s} \ll 1 / \Delta_{0}$, if we move a significant distance $S$ along the Plateau border (comparable with the dimensionless distance $\frac{\pi}{6}$ between the Plateau border entrance and its symmetry point), the boundary condition (25) implies that $\partial^{2} U_{s} / \partial S^{2} \ll 1$, suggesting $\partial U_{s} / \partial S$ is nearly spatially uniform and hence $U_{s}$ vs $S$ is locally quite close to a straight line function. However very near the film-Plateau border junction, equation (30) suggests (in the limit as Cartesian coordinate $X \rightarrow 0$ ) that $\partial^{2} U_{s} / \partial X^{2}$ has a very large value (in turn implying a large $\partial^{2} U_{s} / \partial S^{2}$ since Cartesian coordinate $X$ and arc length coordinate $S$ coincide in the $X \rightarrow 0$ limit). Hence $\partial U_{s} / \partial X$ or equivalently $\partial U_{s} / \partial S$ is spatially nonuniform in this part of the domain. We deduce that the decay of the velocity field $U_{s}$ is 'complex' in the sense that there is a non-uniform decay region near the entrance to the Plateau border, followed by a uniform decay region further along the border.

An asymptotic formulation (equation (30)) in terms of a Cartesian coordinate $X$ (in lieu of an arc length coordinate $S$ ) remains a valid description in the non-uniform region, thereby simplifying the calculations we need to perform there. In what follows we demonstrate that these (asymptotic) governing equations admit power law solutions (see Appendix B.1) and perturbation solutions (see Appendix B.2). The actual solution we seek for $U_{s}$ vs $S$ is obtained via generating two independent solutions (see Appendix B.3) and taking a linear combination of them (see Appendix B.4). An analytic estimate that is consistent with the asymptotic formulation and that determines the ratio between the surface strain rate and surface velocity at the film-Plateau border junction is obtained and discussed in Appendix B.5-Appendix B.6.

\section{Appendix B.1. Power law solutions}

We seek velocity fields on the Plateau border surface $U_{s}$ vs $X$ satisfying equation (30). It is instructive to consider various possible solutions of this equation, even if those we 
generate in the first instance do not have the desired property that they decay to zero at the symmetry point of the Plateau border. Since equation (30) is linear, provided we can find linearly independent solutions of it, linear combinations of those independent solutions can be taken to meet the constraint imposed at the symmetry point.

In the limit where $X \gg \sqrt{\Delta_{0}}$ equation (30) simplifies to

$$
6 U_{s} / X^{2} \approx M_{s} \partial^{2} U_{s} / \partial X^{2}
$$

Assuming an asymptotic solution $U_{s} \sim X^{\Lambda}$, it follows $\Lambda(\Lambda-1)=6 / M_{s}$, and hence

$$
\Lambda=\frac{1}{2} \pm \sqrt{\frac{1}{4}+\frac{6}{M_{s}}}
$$

Since we are interested in large $M_{s}$ values here, we can simplify to

$$
\Lambda \approx 1+6 / M_{s} \text { or } \Lambda \approx-6 / M_{s}
$$

with $6 / M_{s} \ll 1$ in the regime of interest. We conclude that there are two independent modes of behaviour for $U_{s}$. One grows nearly linearly with $X$. The other is almost constant. Subtracting the near linear term from the near constant one, gives a solution that exhibits a nearly uniform decay.

Such a solution could be viewed as a 'frustrated exponential'. Specifically it is 'trying' to decay as an exponential, but as that decay occurs, the Plateau border thickens (i.e. the denominator on the left hand side of equation (30)) grows. This changes the characteristic length scale for the 'exponential' decay, which in this particular case is arrested into a near uniform decay instead of the originally anticipated exponential.

\section{Appendix B.2. Perturbation solutions}

There is an issue with the above power law asymptotic solutions: equation (B.1) only applies for $X \gg \sqrt{\Delta_{0}}$ but does not apply all the way down to $X=0$. In that limit we must return to equation (30). Approximate solutions to (30) can however be obtained by anticipating that on the left hand side of that equation we can replace $U_{s}$ by either a near 
constant solution $\left.U_{s}\right|_{X=0}$ or by a near linear solution $\left(\partial U_{s} /\left.\partial X\right|_{X=0}\right) X$. We then integrate the right hand side of that equation twice to obtain an improved approximation for $U_{s}$.

In the former case (an approximation based on a 'near constant' function) we obtain

$$
\frac{\partial U_{s}}{\partial X} \approx \frac{\left.3 \sqrt{2} U_{s}\right|_{X=0}}{\sqrt{\Delta_{0}} M_{s}} \arctan \left(\frac{X}{\sqrt{2 \Delta_{0}}}\right)
$$

We have (deliberately) set an integration constant here such that equation (B.4) vanishes as $X \rightarrow 0$, to comply with our assumption (for this particular solution branch) that $U_{s}$ should change only very little with respect to $\left.U_{s}\right|_{X=0}$. Integrating again gives

$$
U_{s}-\left.U_{s}\right|_{X=0} \approx \frac{\left.3 \sqrt{2} U_{s}\right|_{X=0}}{\sqrt{\Delta_{0}} M_{s}}\left(X \arctan \left(\frac{X}{\sqrt{2 \Delta_{0}}}\right)-\sqrt{\frac{\Delta_{0}}{2}} \log \left(\frac{X^{2}}{2 \Delta_{0}}+1\right)\right) .
$$

Even though for $X \ll \sqrt{\Delta_{0}}$ this solution has (by construction) only second order variation in $X$, note that for $X \gg \sqrt{\Delta_{0}}$ the expected behaviour is

$$
U_{s}-\left.U_{s}\right|_{X=0} \sim \frac{\left.3 \sqrt{2} U_{s}\right|_{X=0}}{\sqrt{\Delta_{0}} M_{s}} \frac{\pi}{2} X
$$

Our assumption of a 'near constant' function can nevertheless still apply even for values of $X / \sqrt{\Delta_{0}}$ large compared to unity, as long as $X / \sqrt{\Delta_{0}}$ is smaller than $M_{s}$.

In the latter case (basing the approximation on a 'near linear' function substituted into the left hand side of (30)) we obtain

$$
\left.\frac{\partial U_{s}}{\partial X} \approx \frac{\partial U_{s}}{\partial X}\right|_{X=0}\left(1+\frac{3}{M_{s}} \log \left(1+\frac{X^{2}}{2 \Delta_{0}}\right)\right)
$$

and hence

$$
\left.U_{s} \approx \frac{\partial U_{s}}{\partial X}\right|_{X=0}\left(X+\frac{3}{M_{s}}\left(X \log \left(1+\frac{X^{2}}{2 \Delta_{0}}\right)+2 \sqrt{2} \Delta_{0}^{1 / 2} \arctan \left(\frac{X}{\sqrt{2 \Delta_{0}}}\right)-2 X\right)\right)
$$

where we have chosen integration constants that recover the set value of $\partial U_{s} /\left.\partial X\right|_{X=0}$ and that ensure $\left.U_{s}\right|_{X=0}$ vanishes.

Equation (B.8) also has a well defined asymptotic behaviour as $X$ becomes much larger 
than $\Delta_{0}^{1 / 2}$. The function is dominated by the value $\left(\partial U_{s} /\left.\partial X\right|_{X=0}\right) X$ in that case, all other terms (including a slightly awkward logarithmic one) are multiplied by a very small prefactor $3 / M_{s}$ with $M_{s} \gg 1$ here. Such behaviour can be inferred from equation (B.1): as $X$ grows towards the order of unity, it is evident that $\partial^{2} U_{s} / \partial X^{2}$ becomes very small (on the order of $M_{s}^{-1}$ with $M_{s} \gg 1$ ), implying $\partial U_{s} / \partial X$ is uniform.

The observation that the solutions of equation (30) have well defined asymptotic behaviours for $X \gg \sqrt{\Delta_{0}}$ is what allows us to find a combination of the available solutions satisfying a constraint that velocity must vanish on the approach to the symmetry point on the Plateau border. The procedure for doing this is described below.

\section{Appendix B.3. Generating independent solutions}

We can select the required solution via a linear combination method. This involves generating independent solutions of the governing differential equation, in the first instance without taking regard of the actual boundary conditions imposed. The solutions thereby obtained are then combined to satisfy the correct boundary conditions.

Consider two solutions $U_{s(I)}$ and $U_{s(I I)}$ with the following conditions at $X=0$ :

$$
\begin{gathered}
\left.U_{s(I)}\right|_{X=0}=1 \text { and } \partial U_{s(I)} /\left.\partial X\right|_{X=0}=0 \\
\left.U_{s(I I)}\right|_{X=0}=0 \text { and } \partial U_{s(I I)} /\left.\partial X\right|_{X=0}=1 / \sqrt{\Delta_{0} M_{s}} .
\end{gathered}
$$

We integrate both solutions numerically out to values of $X$ much larger than $\sqrt{\Delta_{0}}$. In this regime we expect

$$
\begin{gathered}
U_{s(I)} \sim U_{s(I \infty)}+\left.\frac{\partial U_{s}}{\partial X}\right|_{(I \infty)} X \\
U_{s(I I)} \sim U_{s(I I \infty)}+\left.\frac{\partial U_{s}}{\partial X}\right|_{(I I \infty)} X
\end{gathered}
$$

where $U_{s(I \infty)}, \partial U_{s} /\left.\partial X\right|_{(I \infty)}, U_{s(I I \infty)}$ and $\partial U_{s} /\left.\partial X\right|_{(I I \infty)}$ are constants that we determine numerically. Specifically $\partial U_{s} /\left.\partial X\right|_{(I \infty)}$ and $\partial U_{s} /\left.\partial X\right|_{(I I \infty)}$ are slopes of the $U_{s(I)}$ vs $X$ and $U_{s(I I)}$ vs $X$ curves, whilst $U_{s(I \infty)}$ and $U_{s(I I \infty)}$ are Legendre transforms, i.e. extrapolations of the tangent to those curves back to the intercept on the $U_{s}$ axis. All these numerical 
values are only weakly sensitive to where we terminate the numerical integration, provided (as mentioned before) we integrate out to $X$ values larger than $\sqrt{\Delta_{0}}$.

Functions $U_{s(I)}$ and $U_{s(I I)}$ for $\Delta_{0}=0.00005$ and $M_{s}=100$ are plotted in Figure 15 on the domain $0 \leq X \leq 0.25$. These were computed via a Runge-Kutta integration routine (step size equal to 0.001). The right hand boundary of the integration domain $(X=0.25)$ is chosen arbitrarily to be a value for which the lubrication theory assumptions underlying the derivation of (30) should still apply (specifically in an earlier equation (28) we must constrain the thickness of the Plateau border such that $\Delta \ll 1$ and $\mathrm{d} \Delta / \mathrm{d} X \ll 1)$.

Note that $U_{s(I)}$ is close to the prediction of equation (B.5). This is quite remarkable since the value of the function changes by a factor 3 over the domain plotted, yet equation (B.5) was derived ignoring that variation on the left hand side of (30). Even though we thereby make an error in the numerator of the left hand side of (30), we only do so for $X$ values where the denominator of the left hand side of (30) has increased significantly, making the quotient itself less important.

Moreover we observe that $U_{s(I I)}$ is roughly approximated by a linear function $U_{s(I I)} \approx$ $X / \sqrt{\Delta_{0} M_{s}}$, but equation (B.8) performs better still (being virtually indistinguishable from numerical data). A consequence of $U_{s(I I)}$ being nearly linear is that the Legendre transform $U_{s(I I \infty)}$ is numerically a very small value (certainly much smaller than $\left.U_{s(I \infty)}\right)$.

The analysis leading to equations (B.11)-(B.12) is only strictly valid in the lubrication region where $\Delta \ll 1$ and $\mathrm{d} \Delta / \mathrm{d} X \ll 1$. The arguments however generalise to an arc length coordinate $S$ measured along the Plateau border surface (essentially we replace $\partial^{2} U_{s} / \partial X^{2}$ in equation (30) by $\partial^{2} U_{s} / \partial S^{2}$ ). We deduce analogously (for $S$ values larger than $\sqrt{\Delta_{0}}$ )

$$
\begin{gathered}
U_{s(I)} \sim U_{s(I \infty)}+\partial U_{s} /\left.\partial X\right|_{(I \infty)} S \\
U_{s(I I)} \sim U_{s(I I \infty)}+\partial U_{s} /\left.\partial X\right|_{(I I \infty)} S
\end{gathered}
$$

where the numerical constants $U_{s(I \infty)}, \partial U_{s} /\left.\partial X\right|_{(I \infty)}, U_{s(I I \infty)}$ and $\partial U_{s} /\left.\partial X\right|_{(I I \infty)}$ are identical to those obtained previously. 
Appendix B.4. Linear combination of solutions

We now seek a linear combination (denoted $U_{s(l i n)}$ ) of $U_{s(I)}$ and $U_{s(I I)}$ that vanishes at the symmetry point of the Plateau border (corresponding to $S=\pi / 6$ ).

We first define a parameter $c^{\prime}$

$$
c^{\prime}=\frac{U_{s(I \infty)}+\left.\frac{\partial U_{s}}{\partial X}\right|_{(I \infty)} \frac{\pi}{6}}{U_{s(I I \infty)}+\left.\frac{\partial U_{s}}{\partial X}\right|_{(I I \infty)} \frac{\pi}{6}} .
$$

To ensure that $U_{s(\text { lin })}$ vanishes at $S=\frac{\pi}{6}$ we can define

$$
U_{s(l i n)}=-U_{s(I)}+c^{\prime} U_{s(I I)} .
$$

We have chosen the sign here such that $U_{s(\text { lin })}$ is a negative quantity (which corresponds to surfactant transport in the expected direction from Plateau border to film). In what follows however we consider for convenience the absolute value $\left|U_{s(\text { lin })}\right|$.

Figure 16 shows $\left|U_{s(l i n)}\right|$ obtained via a linear combination of $U_{s(I)}$ and $U_{s(I I)}$ which were themselves shown previously in Figure 15. It is apparent (as has been stated previously) that there is a region where the surface strain rate is non-uniform near the Plateau border entrance, followed by a uniform strain rate region over the rest of the Plateau border surface. The solution for $\left|U_{s(l i n)}\right|$ exhibits a rapid initial decay that is arrested and replaced by a less abrupt straight line decay.

The data on Figure 16 (which have $\Delta_{0}=0.00005$ and $M_{s}=100$ ) compare favourably with the corresponding finite element numerical data obtained from COMSOL as plotted on Figure 8(b): we have not included those COMSOL data on Figure 16, because they are actually so close as to be indistinguishable on the scale of the plot. An approximation to $U_{s(l i n)}$ can also be obtained based on a linear combination of the perturbation approximations in Appendix B.2. This is also plotted in Figure 16: agreement is imperfect ${ }^{14}$.

\footnotetext{
${ }^{14}$ There are some subtleties with the way this approximate solution has been obtained. Given that equations (B.6) and (B.8) were derived in a domain in which Cartesian coordinate $X$ matches arc length $S$, we assumed that we could replace $X$ by $S$ throughout. We then assumed we could take a linear combination that vanished as $S \rightarrow \frac{\pi}{6}$. This approach turns out to be consistent with the results that will be presented in Appendix B.5-Appendix B.6 apart from slight changes in the values of some small logarithmic corrections.
} 
It is now very easy to obtain the ratio between the surface strain rate and surface velocity at the Plateau border entrance (this ratio being necessary to achieve film-Plateau border matching as we have discussed in the main text). By construction, $\left|U_{s(l i n)}\right| X=0 \mid=$ $\left.U_{s(I)}\right|_{X=0}=1$ and $\partial\left|U_{s(l i n)}\right| /\left.\partial X\right|_{X=0}=-c^{\prime} \partial U_{s(I I)} /\left.\partial X\right|_{X=0}=-c^{\prime} / \sqrt{\Delta_{0} M_{s}}$. Hence

$$
\left|U_{s(l i n)}\right|^{-1} \partial\left|U_{s(l i n)}\right| /\left.\partial X\right|_{X=0}=-c^{\prime} / \sqrt{\Delta_{0} M_{s}}
$$

We now identify the parameter $c$ in equation (32) with the parameter $c^{\prime}$ (obtained via equation (B.15)) here. Via equation (23) this parameter governs the extent to which the presence of the Plateau border limits the flow at the film-Plateau border junction.

Values of the parameter $c$ (in the domain $1 \ll M_{s} \ll 1 / \Delta_{0}$ ) obtained via this asymptotic linear combination technique have been tabulated in Table 3. Values of $c$ obtained independently via COMSOL numerical simulation (as discussed in the main text) are also given, and match the values from the asymptotic linear combination technique.

Table 3 shows that $c$ is less than $\sqrt{3}$ in this domain $1 \ll M_{s} \ll 1 / \Delta_{0}$ (the particular value of $\sqrt{3}$ applying instead over the quite different domain $\left.\Delta_{0} \ll M_{s} \ll 1\right)$. The significance of $c$ now being less than $\sqrt{3}$ can be seen in equation (23), which predicts surface velocities 'constrained' by the Plateau border at the film-Plateau border junction to be rather more than half the 'unconstrained' values.

\section{Appendix B.5. Derivation of analytic estimate of $c^{\prime}$}

It is possible to obtain an analytic estimate of $c^{\prime}$ using the perturbation approximations developed in Appendix B.2.

We approximate $U_{s(I)}$ (itself defined in Appendix B.3) by the solution given in equation (B.5) noting that $\left.U_{s(I)}\right|_{X=0}=1$. Based on equations (B.4)-(B.5), the Legendre transform of $U_{s(I)}$ is approximately

$$
U_{s(I)}-X \frac{\partial U_{s(I)}}{\partial X} \approx 1-\frac{3}{M_{s}} \log \left(\frac{X^{2}}{2 \Delta_{0}}+1\right)
$$

We seek the value of the Legendre transform $U_{s(I \infty)}$ for $X \gg \sqrt{\Delta_{0}}$. Equation (B.18) suggests this is sensitive to the $X$ value chosen, although the sensitivity is extremely weak, 
involving a logarithmic correction in $X$ which is moreover multiplied by an order $M_{s}^{-1}$ prefactor, with $M_{s} \gg 1$ here.

We shall take $U_{s(I \infty)}$ to be evaluated at a specific point denoted $X_{R K}$ (chosen here so as to correspond to the right hand end of the Runge-Kutta integration domain that was already employed in Appendix B.3). In our case $X_{R K}=0.25$. Defining the symbol $L_{R K}$ to be $\log \left(1+X_{R K}^{2} /\left(2 \Delta_{0}\right)\right)$, we find (via equation (B.18))

$$
U_{s(I \infty)} \approx 1-\frac{3 L_{R K}}{M_{s}}
$$

The value $\partial U_{s} /\left.\partial X\right|_{(I \infty)}$ (i.e. the $X \gg \sqrt{\Delta_{0}}$ limit of $\left.\partial U_{s(I)} / \partial X\right)$ meanwhile is obtained unambiguously from equation (B.4) to be

$$
\left.\frac{\partial U_{s}}{\partial X}\right|_{(I \infty)} \approx \frac{3 \sqrt{2}}{\sqrt{\Delta_{0}} M_{s}} \frac{\pi}{2} .
$$

We now proceed to approximate $U_{s(I I)}$ by the solution given in equation (B.8) noting from equation (B.10) that $\partial U_{s(I I)} /\left.\partial X\right|_{X=0}=1 / \sqrt{\Delta_{0} M_{s}}$. Observe moreover from equation (B.7) that the value of $\partial U_{s(I I)} / \partial X$ only ever deviates from $\partial U_{s(I I)} /\left.\partial X\right|_{X=0}$ by relative amounts on the order of $M_{s}^{-1}$. Evaluating at $X=X_{R K}$ we deduce in fact that

$$
\left.\frac{\partial U_{s}}{\partial X}\right|_{(I I \infty)} \approx \frac{1}{\sqrt{\Delta_{0} M_{s}}}\left(1+\frac{3 L_{R K}}{M_{s}}\right)
$$

where $L_{R K}$ is the logarithmic term defined above, which as in equation (B.19) is divided through by $M_{s}$ (with $M_{s} \gg 1$ here).

Also observe from equations (B.7)-(B.8) that $U_{s(I I)}-X \partial U_{s(I I)} / \partial X$ (i.e. the Legendre transform) is smaller than $U_{s(I I)}$ itself, by a factor of order $M_{s}^{-1}$. Specifically

$$
U_{s(I I)}-X \frac{\partial U_{s(I I)}}{\partial X} \approx-\frac{1}{\sqrt{\Delta_{0} M_{s}}} \frac{6}{M_{s}}\left(X-\sqrt{2 \Delta_{0}} \arctan \left(\frac{X}{\sqrt{2 \Delta_{0}}}\right)\right) .
$$

Hence evaluating the Legendre transform at $X_{R K}$ we deduce

$$
U_{s(I I \infty)} \approx-\frac{1}{\sqrt{\Delta_{0} M_{s}}} \frac{6}{M_{s}}\left(X_{R K}-\sqrt{2 \Delta_{0}} \frac{\pi}{2}\right) \approx-\frac{6 \sqrt{2}}{M_{s}^{3 / 2}}\left(\frac{X_{R K}}{\sqrt{2 \Delta_{0}}}-\frac{\pi}{2}\right) .
$$


Since $X_{R K}$ is chosen much larger than order $\sqrt{\Delta_{0}}$, this $U_{s(I I \infty)}$ value is clearly sensitive to our choice of $X_{R K}$. Note however that the value of $U_{s(I I \infty)}$ is much smaller than that of $\partial U_{s} /\left.\partial X\right|_{(I I \infty)}$. Moreover it is even smaller than the correction term involving $L_{R K}$ in $\partial U_{s} /\left.\partial X\right|_{(I I \infty)}$ : this is because $X_{R K}$ has a numerical value significantly smaller than unity, whereas $L_{R K}$ (by construction) has a numerical value significantly larger than unity. To a good approximation then we can suppose that $U_{s(I I \infty)}$ vanishes (as would have been the case had $U_{s(I I)}$ been a perfectly linear function in $\left.X\right)$.

Equation (B.15) for $c^{\prime}$ becomes upon substituting from equations (B.19)-(B.21)

$$
c^{\prime} \approx \frac{\left(1-\frac{3 L_{R K}}{M_{s}}+\frac{\sqrt{2}}{\sqrt{\Delta_{0}} M_{s}} \frac{\pi^{2}}{4}\right)}{\frac{\pi}{6} \frac{1}{\sqrt{\Delta_{0} M_{s}}}\left(1+\frac{3 L_{R K}}{M_{s}}\right)} .
$$

Rearranging and Taylor expanding, retaining only leading order terms in the small parameter $L_{R K} / M_{s}$

$$
c^{\prime} \approx \frac{6}{\pi} \sqrt{\Delta_{0} M_{s}}\left(1-\frac{6 L_{R K}}{M_{s}}\right)+\frac{3 \sqrt{2} \pi}{2 \sqrt{M_{s}}}\left(1-\frac{3 L_{R K}}{M_{s}}\right)
$$

This is our approximate analytic expression for $c^{\prime}$, the value $-c^{\prime} / \sqrt{\Delta_{0} M_{s}}$ then fixing (see equation (B.17)) the ratio between the surface strain rate and the surface velocity at the film-Plateau border junction, which then (as alluded to previously) matches the film and Plateau border flows. Implications of equation (B.25) are discussed in the next section.

\section{Appendix B.6. Discussion: Analytic estimate for $c^{\prime}$}

We interpret equation (B.25) as follows. The first term on the right hand side $\frac{6}{\pi} \sqrt{\Delta_{0} M_{s}}$ is actually the value expected when $\Delta_{0} M_{s} \geq O(1)$ (corresponding to velocity decaying uniformly along the entire Plateau border surface). Here however $\Delta_{0} M_{s} \leq O(1)$ and velocity decay is non-uniform, faster near $X=0$ than for larger $X$ values. Thus the right hand side of equation (B.25) for $c^{\prime}$ (which concerns the velocity decay near $X=0$ ) involves a second term, $3 \sqrt{2} \pi /\left(2 \sqrt{M}_{s}\right)$. Each term in equation (B.25) is multiplied by a correction factor, respectively $1-6 L_{R K} / M_{s}$ and $1-3 L_{R K} / M_{s}$, these factors being relatively close to unity ( since $\left.M_{s} \gg 1\right)$. 
Estimates of $c^{\prime}$ computed using equation (B.25) for various $\Delta_{0}$ and $M_{s}$ are shown in Table 3. These are compared with $c$ values obtained numerically with COMSOL (see the main text) and/or by an asymptotic approach (see Appendix B.4).

The table actually reports estimates determined both with and without the logarithmic correction terms (i.e. terms involving $L_{R K} / M_{s}$ in equation (B.25)). For $M_{s}=1000$ and $M_{s}=10000$ the logarithmic corrections make very little difference to the values predicted by equation (B.25) which are generally close to the previously obtained numerical and/or asymptotic values. However values computed with the logarithmic corrections fit the numerical and/or asymptotic data noticeably better than those without. For $M_{s}=100$, equation (B.25) does not perform anywhere near as well as it does for either $M_{s}=1000$ or $M_{s}=10000$. However it is still the case that including the logarithmic corrections represents an improvement over not including them.

It is possible to perform some additional analyses on equation (B.25), supposing (at least as a rough approximation) that the logarithmic correction terms involving $L_{R K} / M_{s}$ may be discarded. We can for instance obtain a minimum value of $c^{\prime}$ for any given $M_{s}$ by taking the limit $\Delta_{0} \rightarrow 0$. The minimum value obtained is

$$
\min _{\Delta_{0}} c^{\prime} \sim 3 \sqrt{2} \pi /\left(2 M_{s}^{1 / 2}\right)
$$

where we assume $M_{s} \gg 1$ and hence $\min _{\Delta_{0}} c^{\prime} \ll 1$. Meanwhile (still assuming terms in $L_{R K} / M_{s}$ are negligible), we obtain a minimum value of $c^{\prime}$ for any given $\Delta_{0}$ by choosing

$$
M_{s}=M_{s(\min )} \equiv \sqrt{2} \pi^{2} /\left(4 \Delta_{0}^{1 / 2}\right)
$$

the minimum then being

$$
\min _{M_{s}} c^{\prime} \sim 2\left(32^{1 / 4} \Delta_{0}^{1 / 4}\right)=2\left(\frac{3 \sqrt{2} \pi}{2 \sqrt{M_{s(\min )}}}\right)
$$

which is twice $\min _{\Delta_{0}} c^{\prime}$ from equation (B.26) (evaluating that equation for $M_{s}=M_{s(\min )}$ ). If $M_{s} \gg M_{s(\min )}$ (or equivalently if $\Delta_{0} \gg \pi^{4} /\left(8 M_{s}^{2}\right)$ ), the value of $c^{\prime}$ is dominated by 
the term involving $\frac{6}{\pi} \sqrt{\Delta_{0} M_{s}}$. If however $M_{s} \ll M_{s(\text { min })}$ (or equivalently $\Delta_{0} \ll \pi^{4} /\left(8 M_{s}^{2}\right)$ ), then $c^{\prime}$ approaches the value $3 \sqrt{2} \pi /\left(2 \sqrt{M_{s}}\right)$ i.e. $\min _{\Delta_{0}} c^{\prime}$ given in equation (B.26).

The data for $M_{s}=1000$ and $M_{s}=10000$ shown in Table 3 actually all have $c^{\prime}$ dominated by the contribution from $\frac{6}{\pi} \sqrt{\Delta_{0} M_{s}}$ because even the smallest $\Delta_{0}$ value in the table (i.e. $5 \times 10^{-5}$ ) already exceeds $\pi^{4} /\left(8 M_{s}^{2}\right)$. Indeed it is only for $M_{s}=100$ and either $\Delta_{0}=5 \times 10^{-5}$ or $\Delta_{0}=5 \times 10^{-4}$ that Table 3 shows $c^{\prime}$ values that are dominated by the term $3 \sqrt{2} \pi /\left(2 \sqrt{M_{s}}\right)$ within equation (B.25).

Finally note that very small $c^{\prime}$ values, such as equation (B.25) predicts for $M_{s} \gg 1$ but $\Delta_{0} \ll 1 / M_{s} \ll 1$, imply that the presence of the Plateau border places very little constraint on the film flow. According to equation (23), the ratio between 'constrained' velocities at the film-Plateau border junction and 'unconstrained' velocities away from that junction would be around $1-c^{\prime} / \sqrt{3}$ for $c^{\prime} \ll 1$.

\title{
Nomenclature
}

\author{
Roman symbols \\ a Plateau border curvature radius \\ $a^{\prime} \quad$ dimensionless $a\left(a^{\prime} \equiv a / L\right)$ \\ $a_{c r i t}^{\prime} \quad$ critical $a^{\prime}$ constraining film-Plateau border junction \\ c dimensionless parameter relating surface strain rate and velocity \\ (at film-Plateau border junction) \\ $c^{\prime}$ coefficient in linear combination (equal to $c$ ) \\ G Gibbs parameter \\ $L \quad$ film half-length \\ $L_{R K} \quad$ a logarithmic correction factor depending on $X_{R K}$ \\ $M_{s} \quad$ rescaled $\bar{\mu}_{s}\left(M_{s}=\bar{\mu}_{s} / a^{\prime}\right)$ \\ $M_{s(\min )} \quad$ value of $M_{s}$ minimising $c^{\prime}$ \\ $\boldsymbol{n}$ unit normal vector \\ $P$ pressure \\ $p$ pressure (made dimensionless on a scale relevant to Plateau border)
}




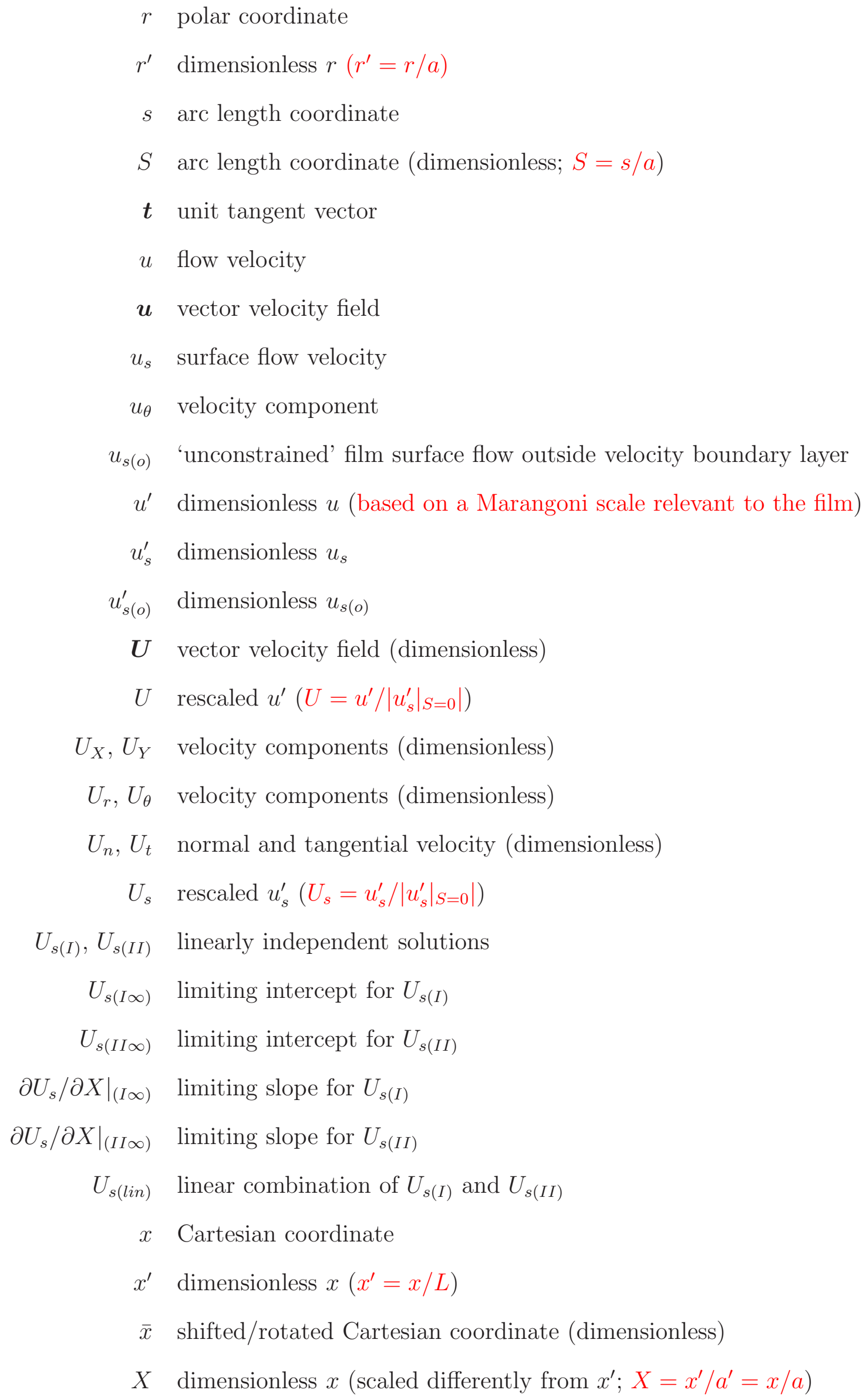




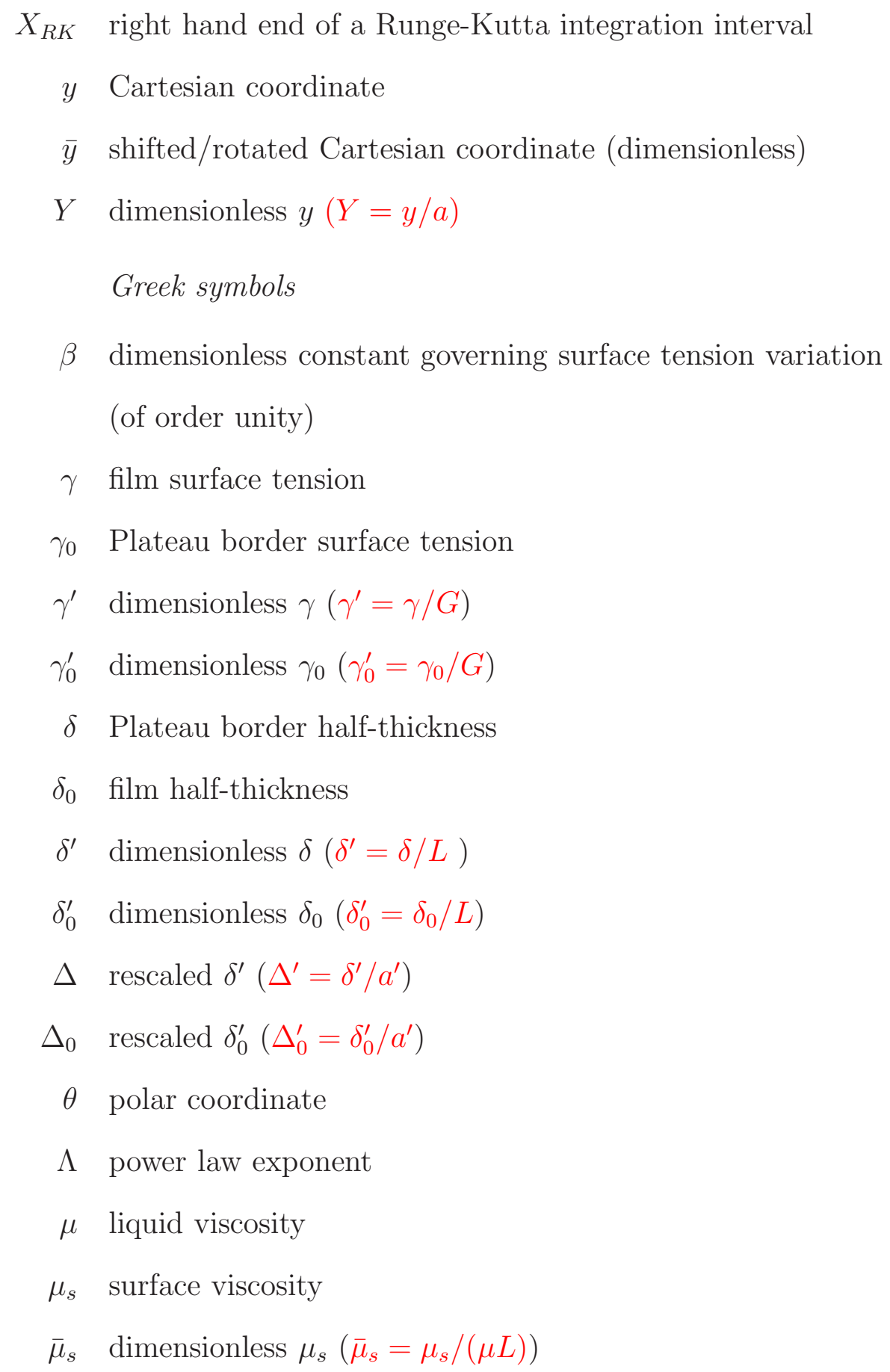

\section{References}

Bretherton, F. P., 1961. The motion of long bubbles in tubes. J. Fluid Mech. 10, 166-188.

Brown, A. K., Kaul, A., Varley, J., 1999. Continuous foaming for protein recovery. Part II. Selective recovery of proteins from binary mixtures. Biotechnol. Bioeng. 62, 291-300. 
Brown, L., Narsimhan, G., Wankat, P. C., 1990. Foam fractionation of globular proteins. Biotechnol. Bioeng. 36, 947-959.

Brunner, C. A., Lemlich, R., 1963. Foam fractionation: Standard separator and refluxing columns. Ind. Eng. Chem. Fundamen. 2, 297-300.

Chang, C.-H., Franses, E. I., 1995. Adsorption dynamics of surfactants at the air/water interface: A critical review of mathematical models, data and mechanisms. Colloids and Surf. A, Physicochem. and Engg Aspects, 1-45.

Durand, M., Stone, H. A., 2006. Relaxation time of the topological T1 process in a twodimensional foam. Phys. Rev. Lett. 97, 226101.

Frankel, S. P., Mysels, K. J., 1962. On the dimpling during the approach of two interfaces. J. Phys. Chem. 66 (1), 190-191.

Grassia, P., Cilliers, J. J., Neethling, S. J., Ventura-Medina, E., 2001. Quasi-onedimensional foam drainage. Eur. Phys. J. E 6, 325-348.

Grassia, P., Homsy, G. M., 1998a. Thermocapillary and buoyant flows with low frequency jitter. I. Jitter confined to the plane. Phys. Fluids 10, 1273-1290.

Grassia, P., Homsy, G. M., 1998b. Thermocapillary and buoyant flows with low frequency jitter. II. Spanwise jitter. Phys. Fluids 10, 1291-1314.

Joye, J. L., Hirasaki, G. J., Miller, C. A., 1992. Dimple formation and behavior during axisymmetrical foam film drainage. Langmuir 8, 3083-3092.

Joye, J. L., Hirasaki, G. J., Miller, C. A., 1994. Asymmetric drainage in foam films. Langmuir 10, 3174-3179.

Joye, J. L., Hirasaki, G. J., Miller, C. A., 1996. Numerical simulation of instability causing asymmetric drainage in foam films. J. Colloid \& Interf. Sci. 177, 542-552.

Lemlich, R., 1968a. Adsorptive bubble separation methods: Foam fractionation and allied techniques. Ind. Eng. Chem. 60, 16-29. 
Lemlich, R., 1968b. Principles of foam fractionation. In: Perry, E. S. (Ed.), Progress in Separation and Purification. Interscience, New York, pp. 1-56.

Lemlich, R., Lavi, E., 1961. Foam fractionation with reflux. Science 134 (3473), 191.

Leonard, R. A., Lemlich, R., 1965. A study of interstitial liquid flow in foam. Part I. Theoretical model and application to foam fractionation. AIChE J. 11, 18-25.

Martin, P. J., Dutton, H. M., Winterburn, J. B., Baker, S., Russell, A. B., 2010. Foam fractionation with reflux. Chem. Engng Sci. 65, 3825-3835.

Ramanan, N., Homsy, G. M., 1994. Linear-stability of a lid-driven cavity flow. Phys. Fluids 6, 2690-2701.

Reinelt, D. A., Kraynik, A. M., 1989. Viscous effects in the rheology of foams and concentrated emulsions. J. Colloid \& Interf. Sci. 132, 491-503.

Reinelt, D. A., Kraynik, A. M., 1990. On the shearing flow of foams and concentrated emulsions. J. Fluid Mech. 215, 431-455.

Richardson, S., 1970. A stick-slip problem related to the motion of a free jet at low Reynolds number. Math. Proc. Cam. Phil. Soc. 67, 477-489.

Schwartz, L. W., Princen, H. M., 1987. A theory of extensional viscosity for flowing foams and concentrated emulsions. J. Colloid \& Interf. Sci. 118, 201-211.

Scriven, L. E., 1960. Dynamics of a fluid interface. Equation of motion for Newtonian surface fluids. Chem. Engng Sci. 12, 98-108.

Smith, M. K., Davis, S. H., 1983. Instabilities of dynamic thermocapillary liquid layers. Part 1. Convective instabilities. J. Fluid Mech. 132, 119-144.

Stevenson, P., 2005. Remarks on the shear viscosity of surfaces stabilised with soluble surfactants. J. Colloid \& Interf. Sci. 290, 603-606.

Stevenson, P., Jameson, G. J., 2007. Modelling continuous foam fractionation with reflux. Chem. Eng. Process. 46, 1286-1291. 
Vitasari, D., 2014. Adsorption and transport of surfactant/protein onto a foam lamella within a foam fractionation column with reflux. PhD thesis, University of Manchester.

Vitasari, D., Grassia, P., Martin, P., 2013a. Simulation of dynamics of adsorption of mixed protein-surfactant on a bubble surface. Colloids and Surf. A, Physicochem. and Engg Aspects 438, 63-73, special Issue: A collection of papers presented at the 9th EUFOAM Conference, Lisbon, Portugal, 8-11 Jul. 2012, edited by M. Emilia Rosa, M. Fátima Vaz and P. Teixeira.

Vitasari, D., Grassia, P., Martin, P., 2015. Surfactant transport onto a foam lamella in the presence of surface viscous stress. Accepted for publication by Appl. Math. Model. doi: 10.1016/j.apm.2015.09.033.

Vitasari, D., Grassia, P., Martin, P. J., 2013b. Surfactant transport onto a foam lamella. Chem. Engng Sci. 102, 405-423.

Weaire, D., Hutzler, S., 1999. The Physics of Foams. Clarendon Press, Oxford. 


\begin{tabular}{|r||rrrr|}
\hline \multicolumn{1}{|c||}{$M_{s}$} & \multicolumn{4}{c|}{$\Delta_{0}$} \\
\hline & $5 \times 10^{-5}$ & $5 \times 10^{-4}$ & $5 \times 10^{-3}$ & $5 \times 10^{-2}$ \\
\hline 0 & $1.077 \times 10^{5}$ & $1.089 \times 10^{4}$ & 1419 & 134.4 \\
$10^{-6}$ & $8.970 \times 10^{4}$ & $1.063 \times 10^{4}$ & 1403 & 134.3 \\
$10^{-5}$ & $5.435 \times 10^{4}$ & 9062 & 1297 & 133.4 \\
$10^{-4}$ & $2.280 \times 10^{4}$ & 5446 & 959.6 & 125.7 \\
$10^{-3}$ & 7675 & 2280 & 547.2 & 96.05 \\
$10^{-2}$ & 2446 & 767.3 & 228.2 & 55.16 \\
$10^{-1}$ & 771.3 & 243.7 & 76.54 & 22.98 \\
1 & 235.6 & 74.51 & 23.57 & 7.522 \\
10 & 58.81 & 18.73 & 6.274 & 2.749 \\
100 & 9.946 & 4.140 & 2.429 & 1.999 \\
1000 & 2.756 & 2.141 & 1.962 & 1.918 \\
$10^{4}$ & 1.994 & 1.933 & 1.915 & 1.910 \\
\hline
\end{tabular}

Table 1: Values of $\left|\partial U_{s} / \partial S\right|_{S=0} \mid$ for various $M_{s}$ and $\Delta_{0}$. Data shown in italics are within $10 \%$ of $6 / \pi$ (the value assumed by Vitasari et al. (2015), corresponding to uniform velocity decay along the entire Plateau border). 


\begin{tabular}{|r||rrrr|}
\hline \multicolumn{1}{|c||}{$M_{s}$} & \multicolumn{4}{c|}{$\Delta_{0}$} \\
\hline & $5 \times 10^{-5}$ & $5 \times 10^{-4}$ & $5 \times 10^{-3}$ & $5 \times 10^{-2}$ \\
\hline 0 & 0 & 0 & 0 & 0 \\
$10^{-6}$ & 0.634 & 0.237 & 0.0992 & 0.0300 \\
$10^{-5}$ & 1.215 & 0.640 & 0.290 & 0.0943 \\
$10^{-4}$ & $\mathbf{1 . 6 1 2}$ & 1.217 & 0.678 & 0.281 \\
$10^{-3}$ & $\mathbf{1 . 7 1 6}$ & $\mathbf{1 . 6 1 2}$ & 1.223 & 0.679 \\
$10^{-2}$ & $\mathbf{1 . 7 2 9}$ & $\mathbf{1 . 7 1 5}$ & $\mathbf{1 . 6 1 3}$ & 1.223 \\
$10^{-1}$ & $\mathbf{1 . 7 2 4}$ & $\mathbf{1 . 7 2 3}$ & $\mathbf{1 . 7 1 1}$ & $\mathbf{1 . 6 2 4}$ \\
1 & $\mathbf{1 . 6 6 5}$ & $\mathbf{1 . 6 6 6}$ & $\mathbf{1 . 6 6 6}$ & $\mathbf{1 . 6 8 1}$ \\
10 & 1.315 & 1.324 & 1.402 & 1.943 \\
100 & 0.703 & 0.925 & 1.717 & 4.469 \\
1000 & 0.616 & 1.513 & 4.387 & 13.56 \\
$10^{4}$ & 1.409 & 4.322 & 13.54 & 42.70 \\
\hline
\end{tabular}

Table 2: Values of the parameter $c$ for various $M_{s}$ and $\Delta_{0}$. Here $c$ has been obtained from $\left|\partial U_{s} / \partial S\right|_{S=0} \mid$ by multiplying through by $\sqrt{\Delta_{0} M_{s}}$. Near the top of the table, the values of $c$ are small (in fact $c$ is identically zero for $M_{s}=0$ ). Note however that there is a band of values in the middle of the table (shown in bold face) where $c$ is within $10 \%$ of the value $\sqrt{3}$. These data correspond to $\Delta_{0} \leq M_{s} \leq 1$. Values in italics towards the bottom part of the table, correspond to those also given in italics in the previous table. Since we already know that $\left|\partial U_{s} / \partial S\right|_{S=0} \mid$ is nearly $6 / \pi$ for those cases, we automatically know that $c \approx \frac{6}{\pi} \sqrt{\Delta_{0} M_{s}}$. For such cases, the value of $c$ then tends to be rather larger than unity, since those data correspond to larger values of $\sqrt{\Delta_{0} M_{s}}$. Below the data shown in bold but above the data shown in italics, there are a set of $c$ values which are significantly less than $\sqrt{3}$. Such data correspond to $M_{s} \geq 1$ but $\Delta_{0} M_{s} \leq 1$. 


\begin{tabular}{|r|r||rrr|}
\hline$\Delta_{0}$ & $M_{s}$ & $c$ (numerical) & $c$ (asymptotic) & $c$ (approximate) \\
\hline $5 \times 10^{-5}$ & 100 & 0.703 & 0.702 & $0.620(0.801)$ \\
& 1000 & 0.616 & 0.615 & $0.617(0.637)$ \\
& 10000 & 1.409 & 1.410 & $1.411(1.417)$ \\
\hline $5 \times 10^{-4}$ & 100 & 0.925 & 0.922 & $0.904(1.093)$ \\
& 1000 & 1.513 & 1.513 & $1.524(1.561)$ \\
\hline 0.005 & 100 & 1.717 & $1.70^{r} 7$ & $1.816(2.016)$ \\
\hline
\end{tabular}

Table 3: Comparison between the values of the parameter $c$ obtained from numerical calculation with COMSOL (as outlined in Sections 7 and 8.3), from asymptotic analysis (as described in Appendix B.3Appendix B.4) and from an approximate analytic formula (given by equation (B.25) in Appendix B.5). The asymptotic analysis and approximate formula are relevant to values of $M_{s}$ significantly larger than unity, but with values of $\Delta_{0} M_{s}$ smaller than unity, and the set of values of $M_{s}$ and $\Delta_{0}$ shown here satisfy those constraints. Two computed values are shown associated with the approximate analytic formula. The first value includes a logarithmic correction (the term $L_{R K}$ in equation (B.25)). The second value (shown in parenthesis) ignores those logarithmic corrections. Throughout the table, values highlighted in italics are within $10 \%$ of what is predicted by the numerical calculations in COMSOL. 
(a)

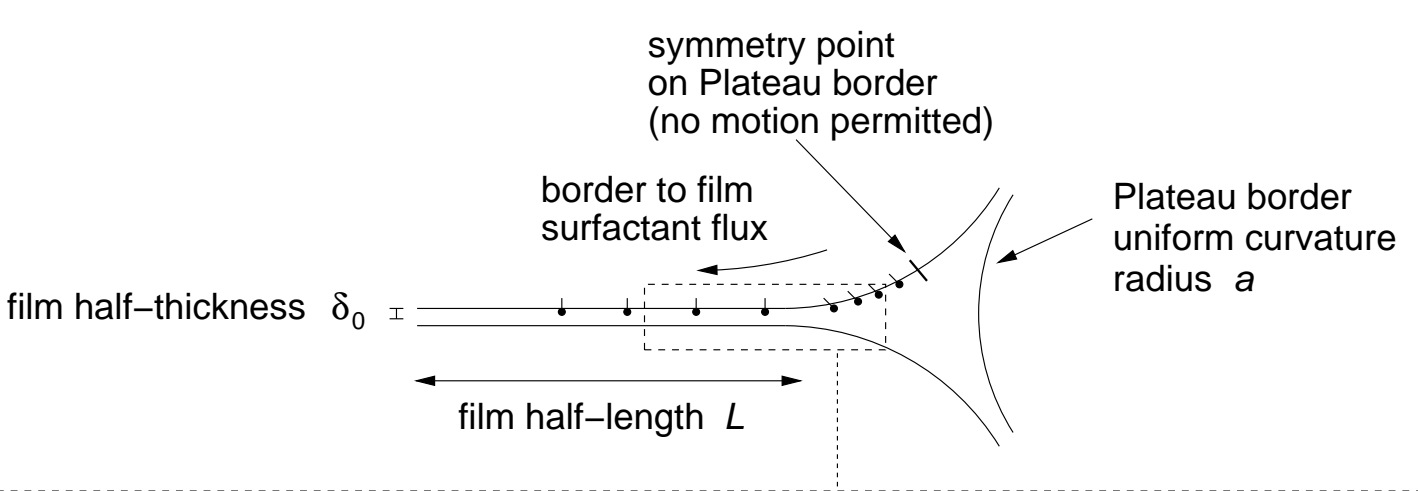

(b)

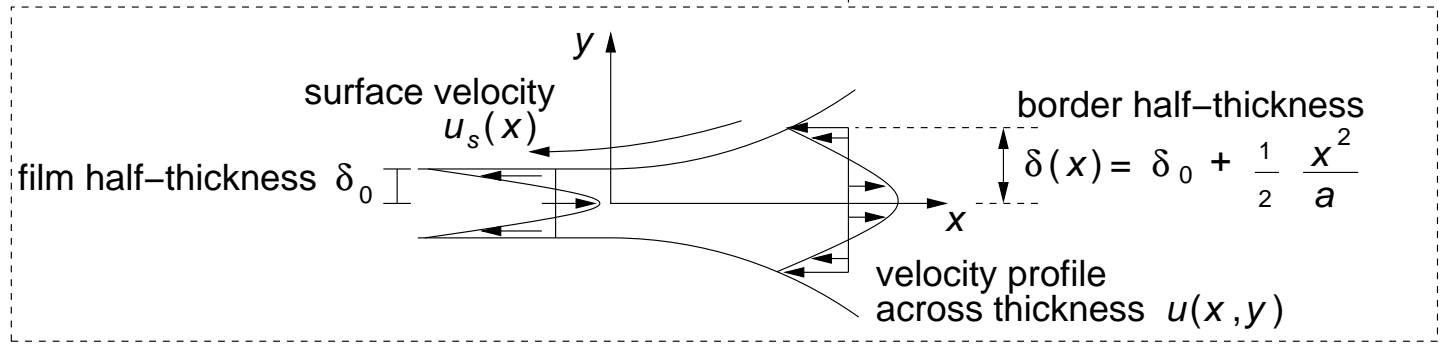

Figure 1: (a) Sketch of a film joining up with a Plateau border during foam fractionation, idealising the film as flat and the Plateau borders as uniform curvature arcs. We are interested in the surfactant flow between the border (higher surfactant coverage and hence lower surface tension) and the film (lower surfactant coverage and hence higher surface tension). The border also has a symmetry point at which no flow may occur. (b) Close up zoomed view of the entrance region of a Plateau border, near the junction between the film and the Plateau border. 


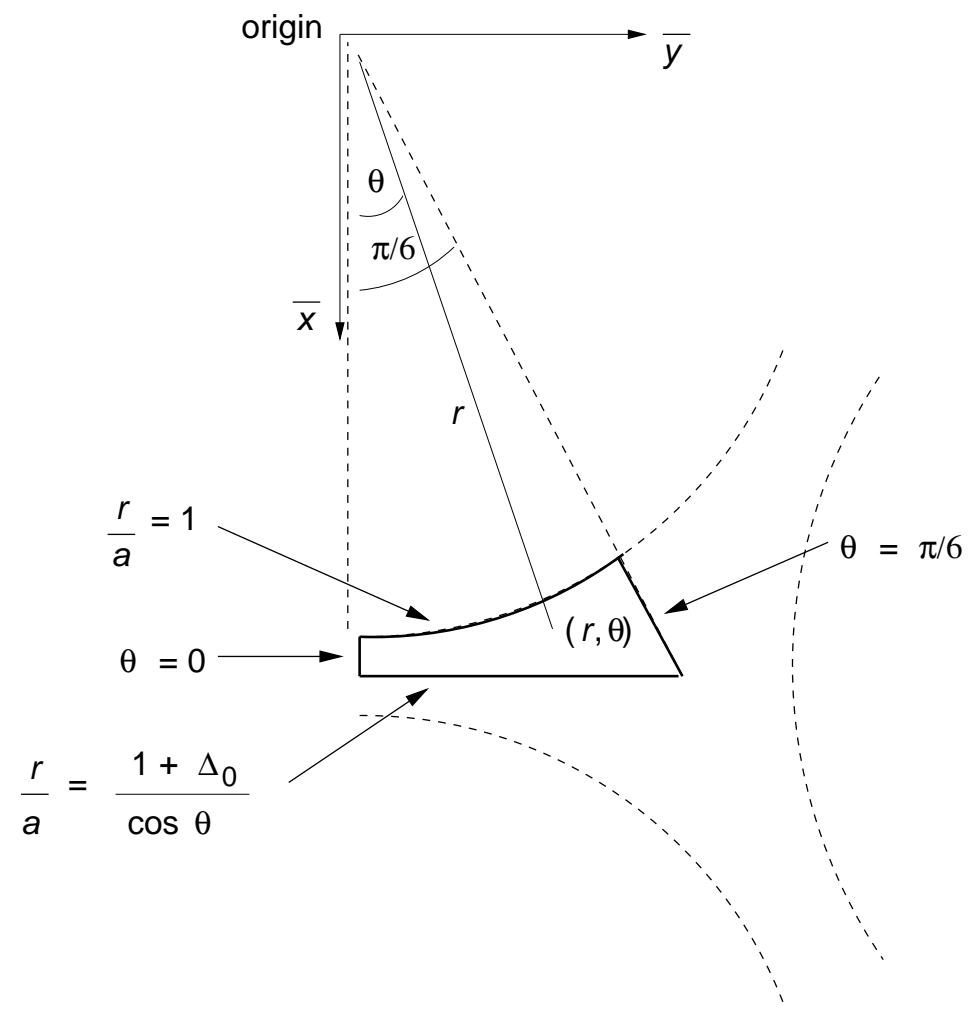

Figure 2: The polar coordinate solution domain corresponding to one sixth of the tricuspid Plateau border (the solution throughout the remainder of the Plateau border being deduced via symmetry). As drawn, the Plateau border radius of curvature is $a$, but coordinates can also be made dimensionless such that the Plateau border curvature radius is scaled to unity, and the film half-thickness is $\Delta_{0}$. The origin is placed outside the Plateau border itself (at the centre of curvature of the Plateau border arc). The domain of interest is $0 \leq \theta \leq \frac{\pi}{6}$ and $1 \leq r / a \leq\left(1+\Delta_{0}\right) / \cos \theta$. Dimensionless Cartesian coordinates $\bar{x}=a^{-1} r \cos \theta$ and $\bar{y}=a^{-1} r \sin \theta$ can also be defined . 

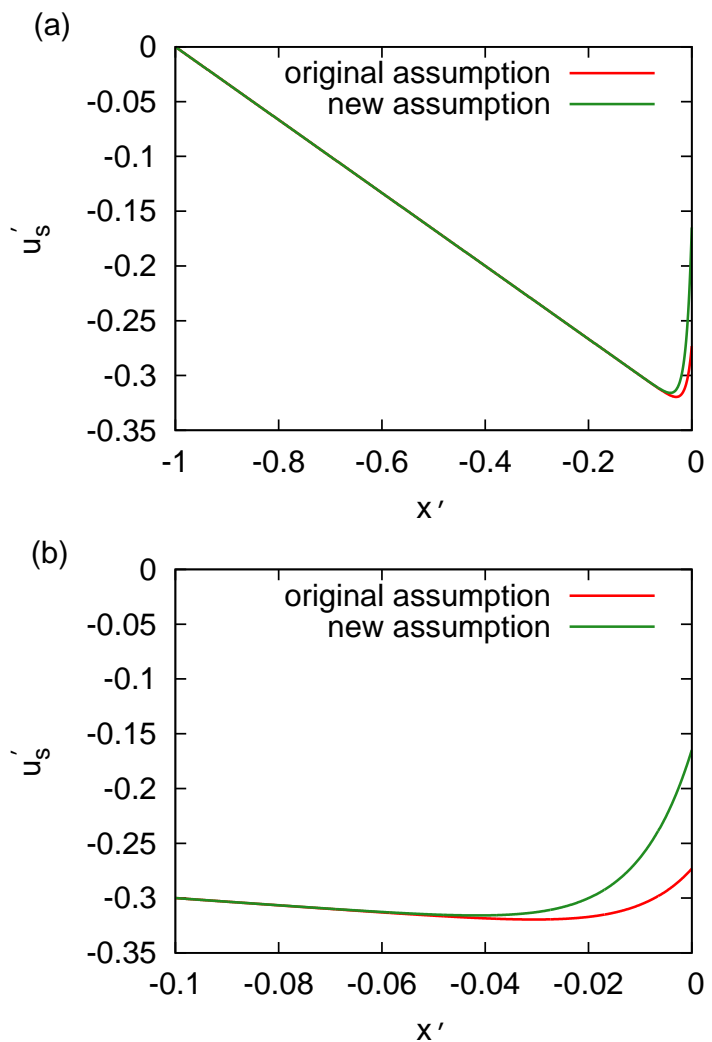

Figure 3: (a) Dimensionless fluid surface velocity $u_{s}^{\prime}$ on a film vs dimensionless coordinate $x^{\prime}$ along the film predicted by equation (34) (the 'original assumption') and equation (36) (the 'new assumption') respectively. We suppose $\bar{\mu}_{s}=0.088$ and $\delta_{0}^{\prime}=4 \times 10^{-3}$ from which we can deduce $\left(\delta_{0}^{\prime} \bar{\mu}_{s} / 3\right)^{1 / 2} \approx 0.010$ and $a_{\text {crit }}^{\prime}=\frac{6}{\pi}\left(\delta_{0}^{\prime} \bar{\mu}_{s} / 3\right)^{1 / 2} \approx 0.020$. We also take $a^{\prime}=0.1$ and set (without loss of generality) $\beta=1$. In equation (36), the parameter $c$ is arbitrarily set to $\sqrt{3}$. (b) A zoomed view of $u_{s}^{\prime}$ vs $x^{\prime}$ in the neighbourhood of $x^{\prime}=0$ where the film joins the Plateau border. 


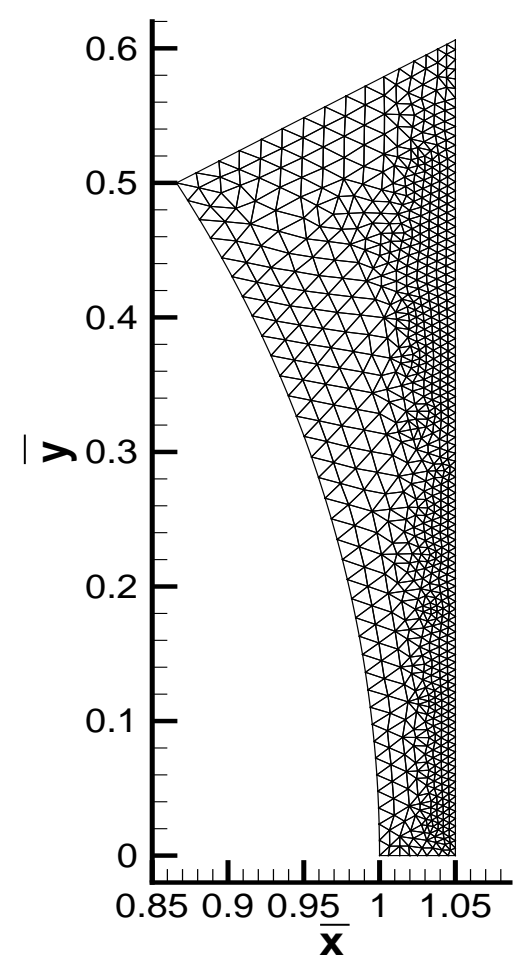

Figure 4: A mesh generated by COMSOL containing 1558 elements in the case where $\Delta_{0}=0.05$ (shown here for illustrative purposes; in fact our calculations were done with a much denser mesh: 191503 elements in the case $\left.\Delta_{0}=0.05\right)$. 
(a)

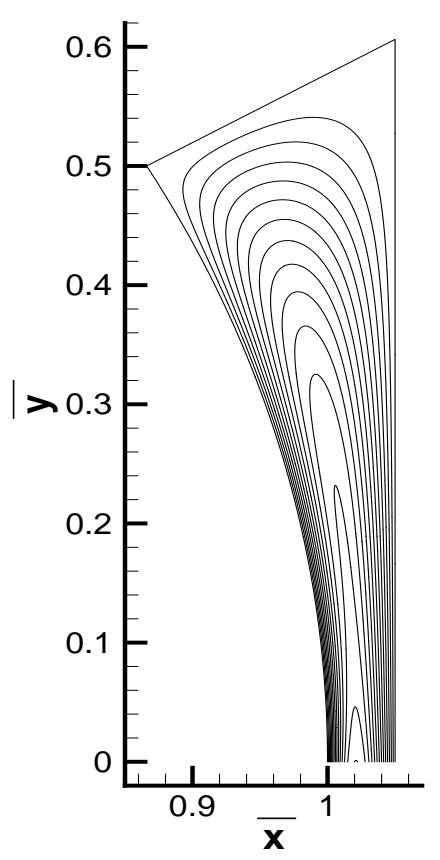

(b)

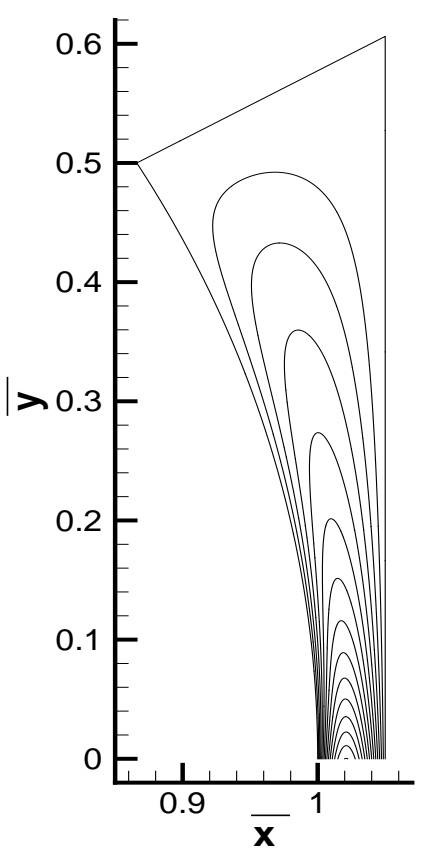

(c)

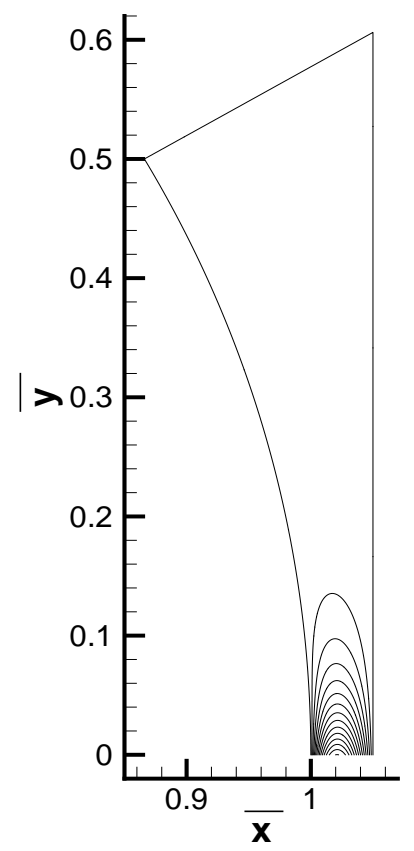

Figure 5: Streamline patterns within the Plateau border in the case $\Delta_{0}=0.05$ and (a) $M_{s}=10^{4}$, (b) $M_{s}=1$ and (c) $M_{s}=0.1$. As the product, $\Delta_{0} M_{s}$ decreases, the flow field decays more rapidly moving into the Plateau border. The streamline patterns were obtained in polar coordinates, but have been converted here onto Cartesian $\bar{x}$ and $\bar{y}$ axes. 
(a)

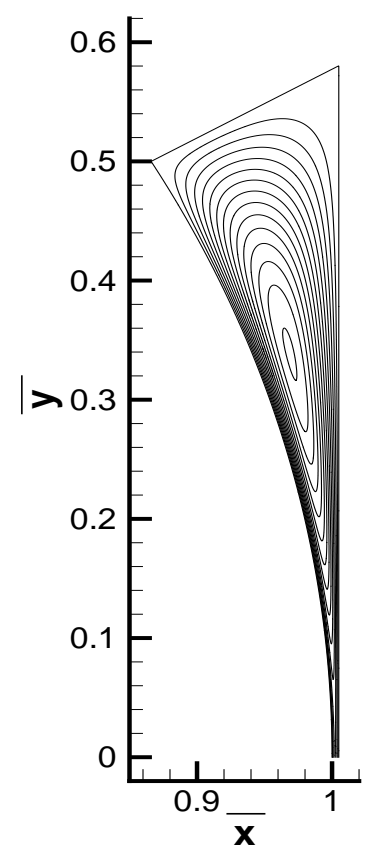

(b)

(c)

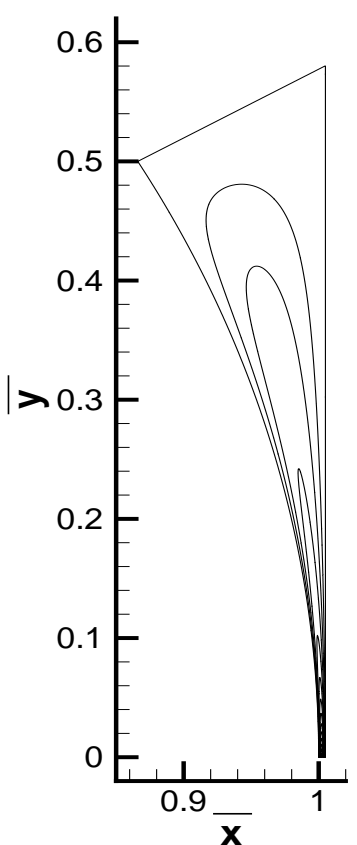

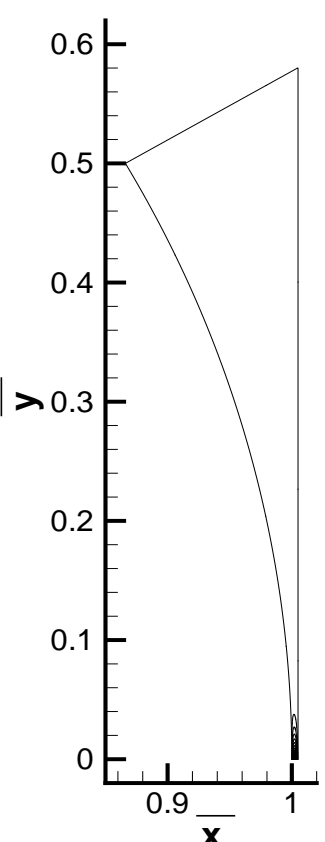

Figure 6: Streamline patterns within the Plateau border in the case $\Delta_{0}=0.005$ and (a) $M_{s}=10^{4}$, (b) $M_{s}=1$ and (c) $M_{s}=0.1$. 
(a) No stagnation point

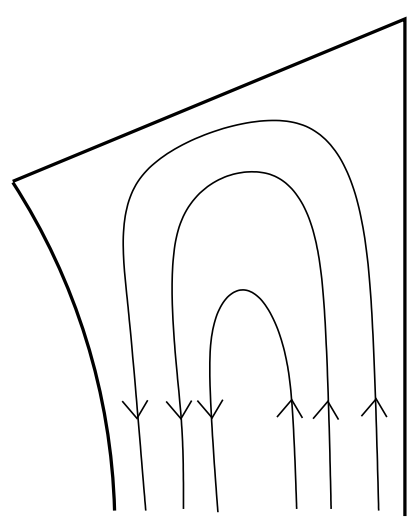

(b) With stagnation points

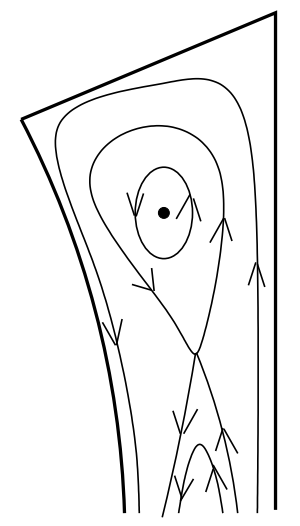

Figure 7: Topology of the streamline pattern in a Plateau border in the case (a) with no stagnation point and (b) with two stagnation points (one centre and one saddle). To aid clarity, the thickness of the Plateau border has been exaggerated in both (a) and (b), instead of drawing the figure to scale. However (b) is deliberately drawn thinner than (a) because cases with low film thickness (and also high surface viscosity) tend to exhibit the stagnation point topology. 

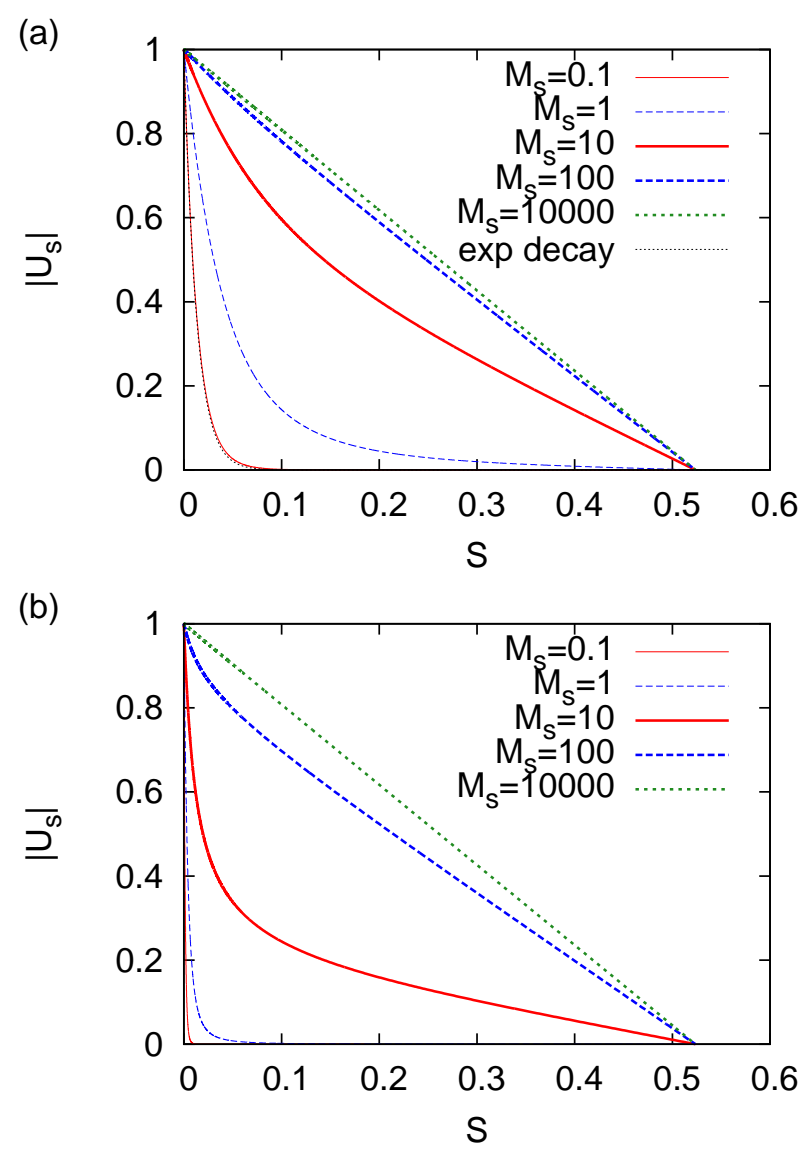

Figure 8: Numerical results for tangential surface flow $\left|U_{s}\right|$ vs distance $S$ along the Plateau border for various surface viscosities $M_{s}$ and for film thickness (a) $\Delta_{0}=0.005$ and (b) $\Delta_{0}=0.00005$. An exponential decay curve $\exp \left(-S / \sqrt{\Delta_{0} M_{s} / 3}\right)$ applies when $\Delta_{0} \ll M_{s} \ll 1$. This curve (labelled 'exp decay') is shown explicitly in (a) for the case $\Delta_{0}=0.005$ and $M_{s}=0.1$ but is barely distinguishable from the numerical data. The analogous exponential curve is not however shown on (b) (which has a much smaller $\Delta_{0}$ and thereby substantially faster decays) to avoid crowding the figure. 


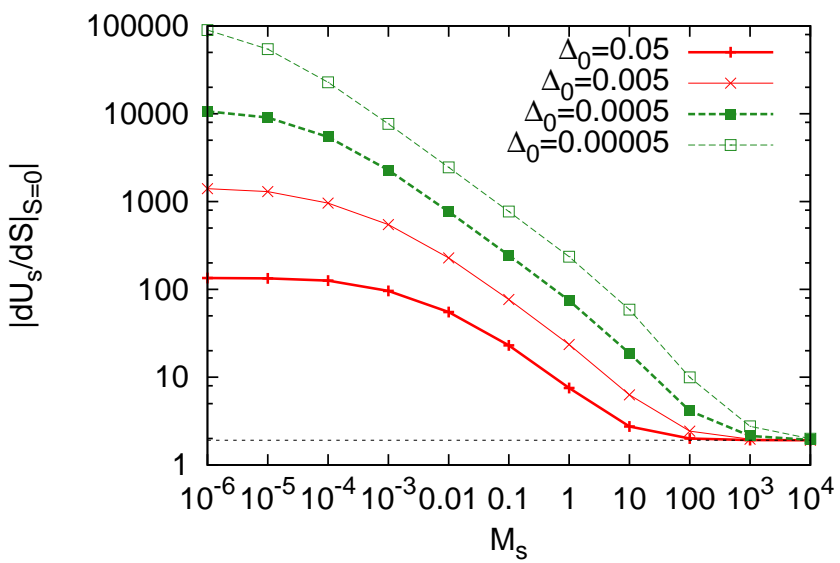

Figure 9: The surface strain rate evaluated at the film-Plateau border junction $\left|\partial U_{s} / \partial S\right|_{S=0} \mid$ (determined from COMSOL numerical simulations in the Plateau border) plotted for various dimensionless film thicknesses $\Delta_{0}$ and various dimensionless surface viscosities $M_{s}$. Knowing this surface strain rate value is relevant to coupling the film and Plateau border flows together. For sufficiently large $M_{s}$, the values of $\left|\partial U_{s} / \partial S\right|_{S=0} \mid$ converge to $6 / \pi$ (horizontal line) with faster convergence seen for larger $\Delta_{0}$. This limiting value corresponds to a uniform velocity decay along the Plateau border surface, which has a dimensionless arc length of $\pi / 6$ between the film-Plateau border junction (with unit velocity in the present scaling) and a symmetry point (with zero velocity) on the border surface. 


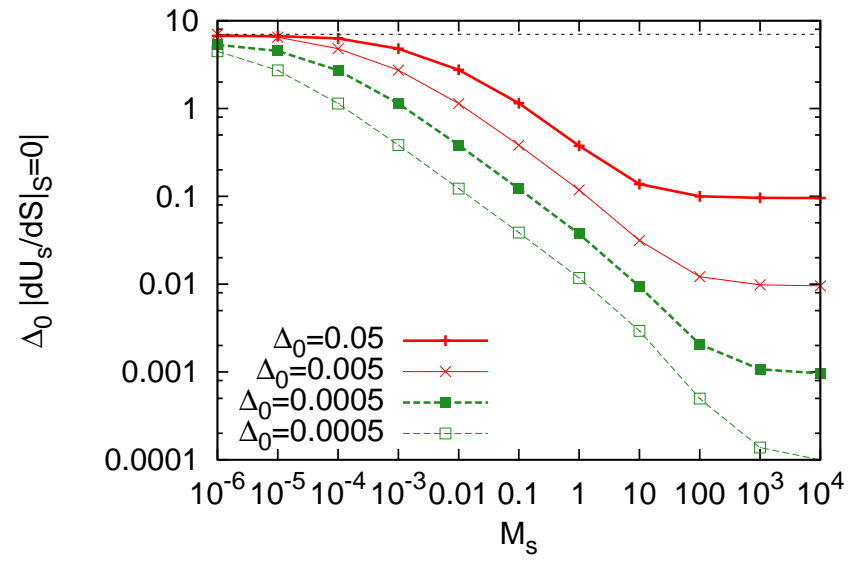

Figure 10: Computed data $\Delta_{0}\left|\partial U_{s} / \partial S\right|_{S=0} \mid$ for various $\Delta_{0}$ and $M_{s}$. In the limit of $M_{s} \rightarrow 0$, $\Delta_{0}\left|\partial U_{s} / \partial S\right|_{S=0} \mid$ seems to converge to a constant (with a value of roughly 7 , shown as a horizontal line). This implies a velocity field decaying along the border over a very small distance of order $\Delta_{0}$. 


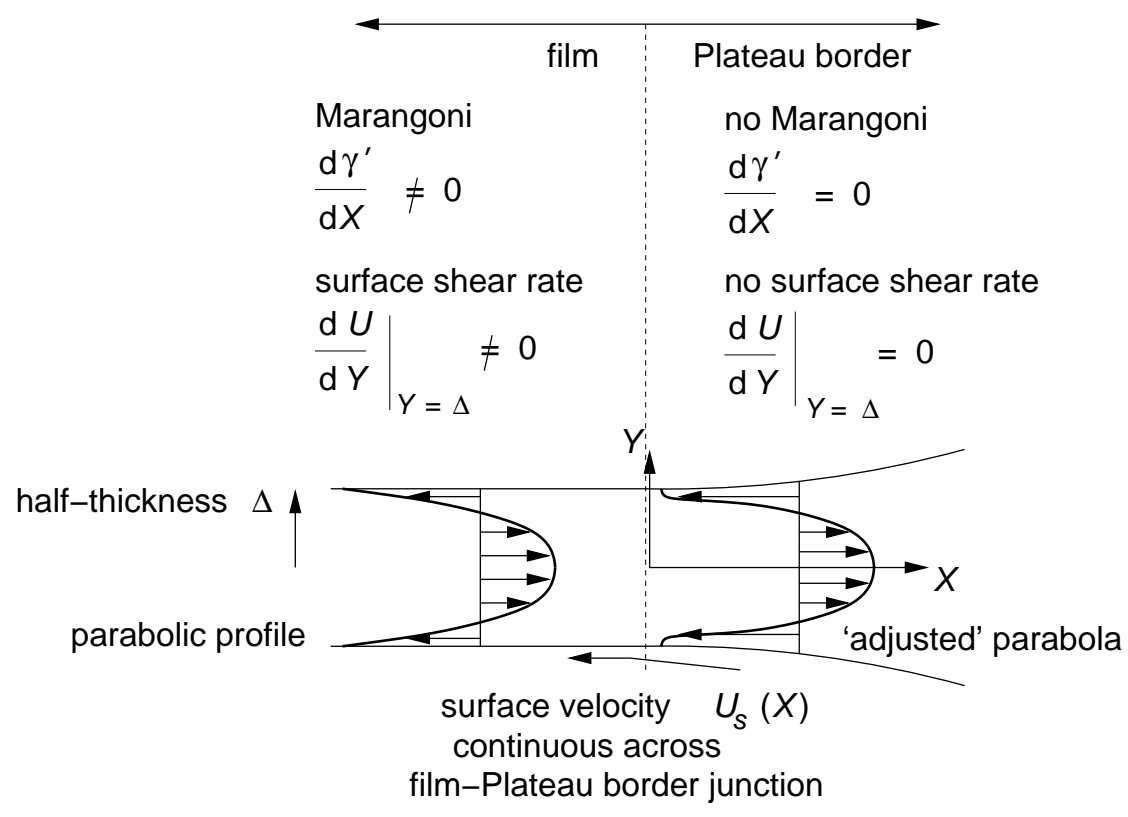

Figure 11: Sketch of a 'stick-slip' singularity, where a parabolic velocity profile $U$ (induced by Marangoni stresses $\partial \gamma^{\prime} / \partial X$ ) in a film, exhibits a local adjustment at the surface of a Plateau border (with neither Marangoni stresses nor surface viscosity). The surface velocity $U_{s}$ is continuous across the film-Plateau border junction, but the surface shear rate $\partial U /\left.\partial Y\right|_{Y=\Delta}$ is not (at least in this idealised case where surface viscous effects are neglected). Such solutions are mathematically possible, but physically are unlikely (as they assume Marangoni stresses decay over exceedingly small length scales). 


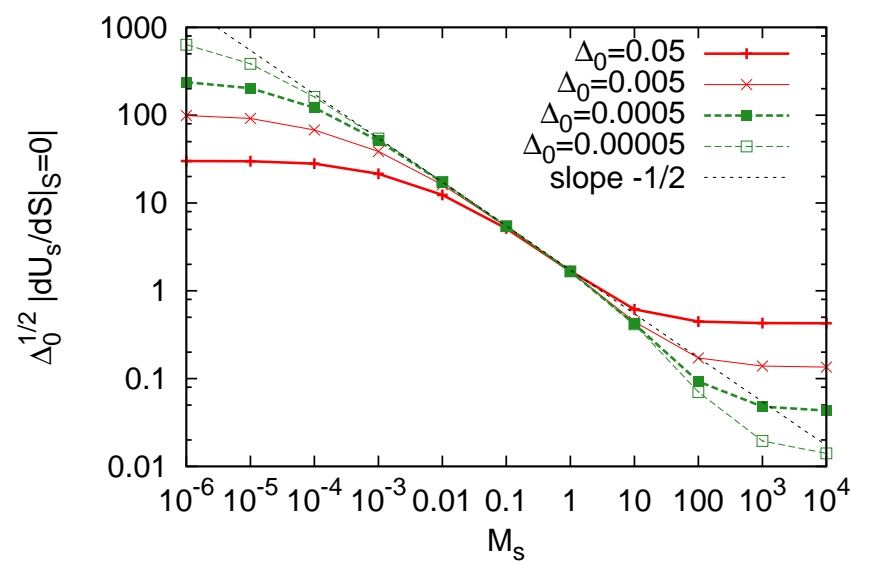

Figure 12: Computed data $\Delta_{0}^{1 / 2}\left|\partial U_{s} / \partial S\right|_{S=0} \mid$ for various $\Delta_{0}$ and $M_{s}$. The line marked 'slope $-\frac{1}{2}$ ' corresponds to $\sqrt{3} M_{s}^{-1 / 2}$. Data for $M_{s}$ values satisfying $\Delta_{0} \leq M_{s} \leq 1$ tend to converge to this line. 


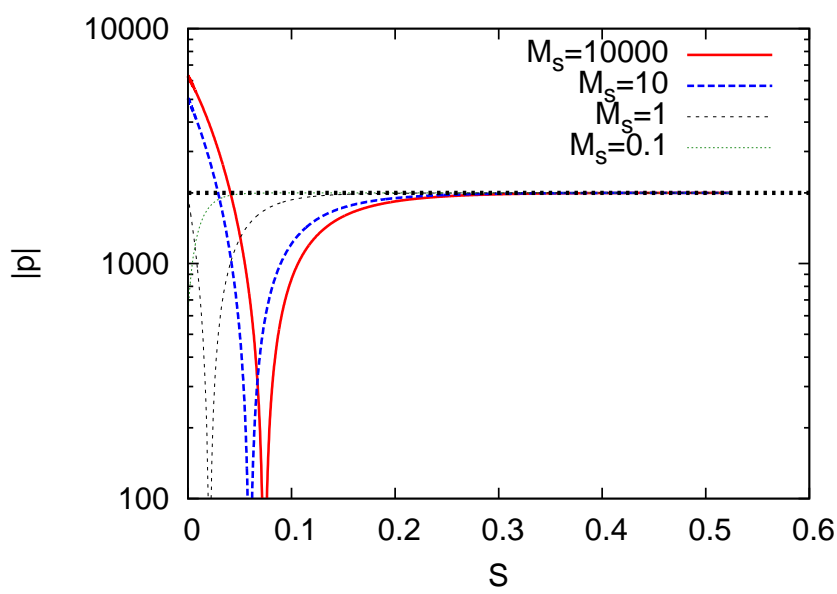

Figure 13: Absolute value of dimensionless pressure $|p|$ vs dimensionless distance $S$ along the Plateau border for dimensionless film thickness $\Delta_{0}=0.005$ and dimensionless surface viscosity $M_{s}$ values 10000 , 10, 1 and 0.1 . The absolute value $|p|=\left(\Delta_{0} a^{\prime}\right)^{-1}$ (shown by the horizontal dotted line) is set at $S=\pi / 6$ (with $a^{\prime}=0.1$ here). This represents the estimated pressure jump between gas and liquid at the Plateau border surface. For $S$ values such that $|p|$ lies near this dotted line, the change in pressure along the surface is relatively modest, making it reasonable to treat the Plateau border as a uniform circular arc in that region. 


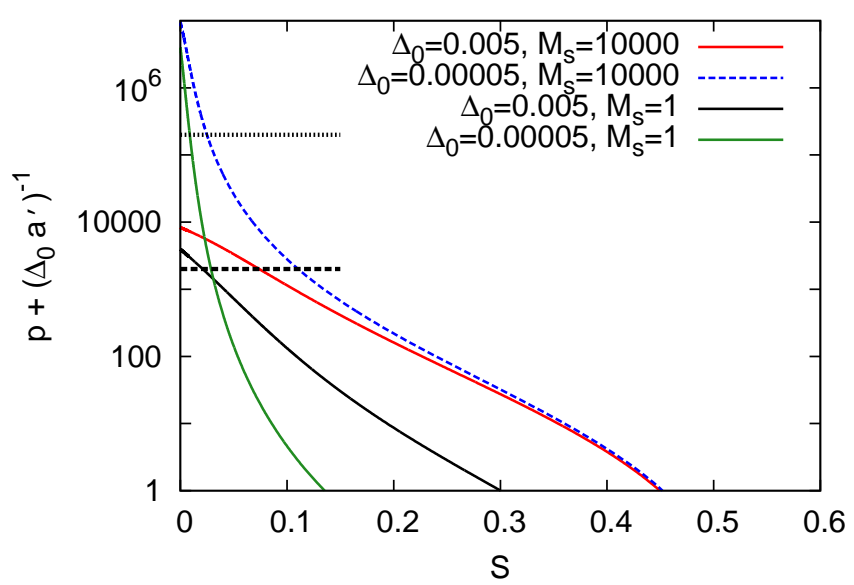

Figure 14: Dimensionless pressure $p+\left(\Delta_{0} a^{\prime}\right)^{-1}$ vs dimensionless distance $S$ along the Plateau border for two different dimensionless film thicknesses $\Delta_{0}=0.005$ and $\Delta_{0}=0.00005$ and two different dimensionless surface viscosities $M_{s}=10000$ and $M_{s}=1$. By construction $p+\left(\Delta_{0} a^{\prime}\right)^{-1} \rightarrow 0$ as $S \rightarrow \frac{\pi}{6}$. The horizontal dotted and dashed lines correspond to vanishing $p$ values, so that $p+\left(\Delta_{0} a^{\prime}\right)^{-1}$ reduces to $\left(\Delta_{0} a^{\prime}\right)^{-1}$ with two different $\Delta_{0}$ values and with $a^{\prime}=0.1$. 

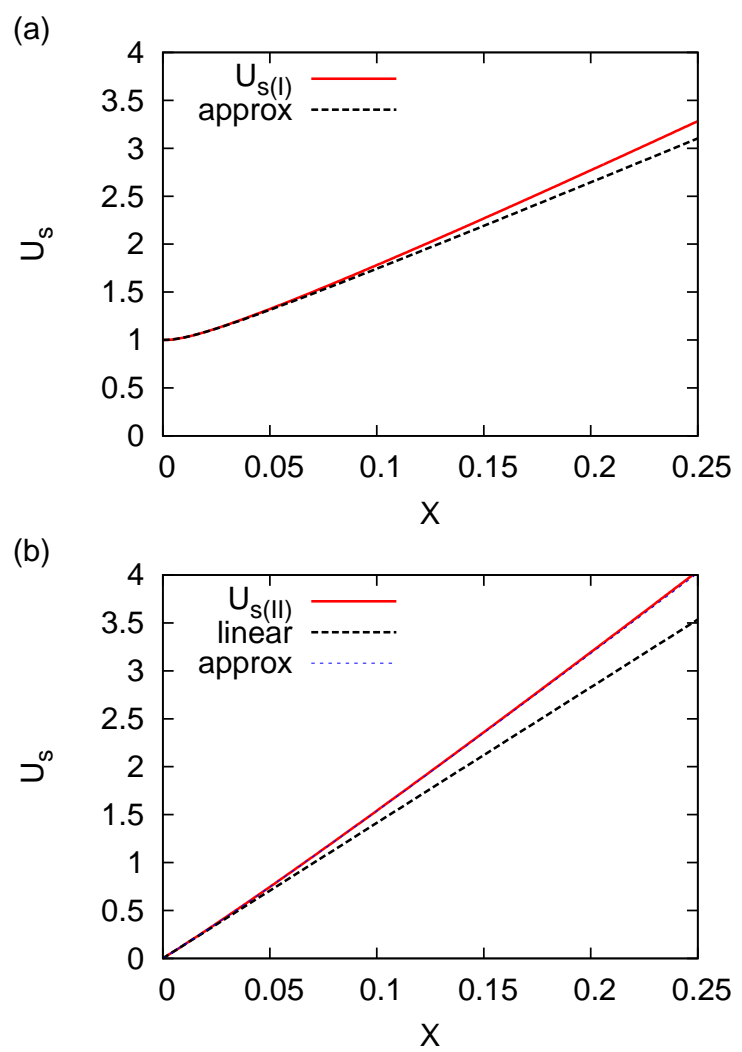

Figure 15: Solutions of equation (30) which has been obtained via an asymptotic formulation for (a) $U_{s(I)}$ and (b) $U_{s(I I)}$ in the case $\Delta_{0}=0.00005$ and $M_{s}=100$. In (a) the approximate formula equation (B.5) is shown. In (b) we compare with a linear function $X / \sqrt{\Delta_{0} M_{s}}$ and also with the approximate formula equation (B.8) (the latter being virtually indistinguishable from $U_{s(I I)}$ ). 


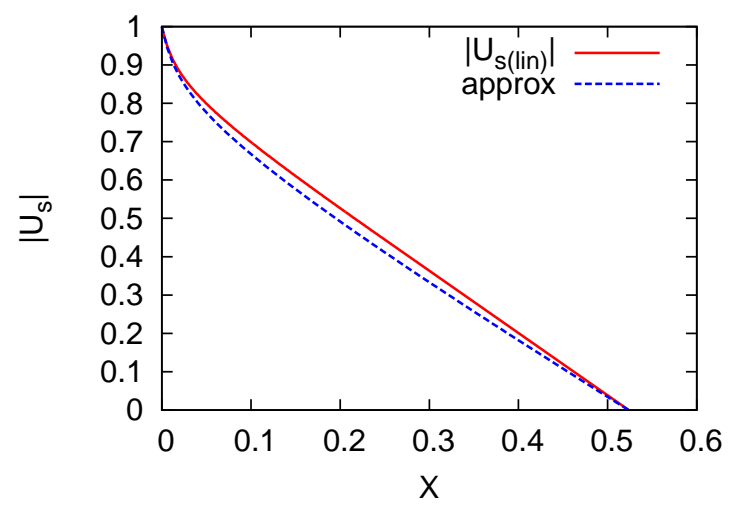

Figure 16: Solutions of equation (30) for the flow speed along the Plateau border surface $\left|U_{s(\text { lin) }}\right|$ (obtained via a linear combination of $U_{s(I)}$ and $U_{s(I I)}$ themselves being solutions of equation (30)) in the case $\Delta_{0}=0.00005$ and $M_{s}=100$. It is also possible to determine the speeds directly via a COMSOL numerical simulation: the COMSOL data (see Figure 8(b)) are not shown explicitly on the current plot as they would be indistinguishable from the data already shown. An approximation to $U_{s(\text { lin })}$ constructed from the perturbation solutions presented in Appendix B.2 is also shown for comparison. 